\title{
Real analytic parameter dependence of solutions of differential equations
}

\section{Paweł Domański}

\begin{abstract}
We consider the problem of real analytic parameter dependence of solutions of the linear partial differential equation $P(D) u=f$, i.e., the question if for every family $\left(f_{\lambda}\right) \subseteq \mathscr{D}^{\prime}(\Omega)$ of distributions depending in a real analytic way on $\lambda \in U, U$ a real analytic manifold, there is a family of solutions $\left(u_{\lambda}\right) \subseteq \mathscr{D}^{\prime}(\Omega)$ also depending analytically on $\lambda$ such that

$$
P(D) u_{\lambda}=f_{\lambda} \quad \text { for every } \lambda \in U,
$$

where $\Omega \subseteq \mathbb{R}^{d}$ an open set. For general surjective variable coefficients operators or operators acting on currents over a smooth manifold we give a solution in terms of an abstract "Hadamard three circle property" for the kernel of the operator. The obtained condition is evaluated giving the full solution (usually in terms of the symbol) for operators with constant coefficients and open (convex) $\Omega \subseteq \mathbb{R}^{d}$ if $P(D)$ is of one of the following types: 1) elliptic, 2) hypoelliptic, 3) homogeneous, 4) of two variables, 5) of order two or 6) if $P(D)$ is the system of Cauchy-Riemann equations. An analogous problem is solved for convolution operators of one variable. In all enumerated cases, it follows that the solution is in the affirmative if and only if $P(D)$ has a linear continuous right inverse which shows a striking difference comparing with analogous smooth or holomorphic parameter dependence problems.
\end{abstract}

2000 Mathematics Subject Classification: Primary 35B30, 35E20, 46F05. Secondary 32U05, 58A25, 46A63, 46A13, 46E10, 46M18.

Keywords: Analytic dependence on parameters, linear partial differential operator, convolution operator, linear partial differential equation with constant coefficients, injective tensor product, surjectivity of tensorized operators, space of distributions, currents, space of ultradistributions in the sense of Beurling, functor Proj ${ }^{1}$, PLS-space, locally convex space, vector valued equation, solvability. 
The paper contains the whole theory working also for operators on Beurling ultradistributions $\mathscr{D}_{(\omega)}^{\prime}$. We prove a characterization of surjectivity of tensor products of general surjective linear operators on a wide class of spaces containing most of the natural spaces of classical analysis.

\section{Introduction}

The problem of parameter dependence of solutions of linear partial differential equations is a classical question. As examples of important contributions to that topic we should mention papers of Tréves [72, 73] and Browder [13]. Later the problem of Hörmander on holomorphic parameter dependence of fundamental solutions was solved in [44], [45] and [46]. Tréves studied smooth and holomorphic dependence. Browder considered real analytic dependence - their results form the first source of motivation for our paper.

Browder looked at a differential operator as a densely defined map on a Hilbert space $L_{2}(\Omega)$. In that case there is no ambiguity in saying "analytically dependent" since all reasonable definitions of Banach valued real analytic functions coincide [1].

We will consider differential operators acting on the space of distributions. In that case we have to explain what does it precisely mean "depending in an analytic way" capturing the intuitive idea of a distribution given by a formula depending analytically on parameters $\lambda$. That idea becomes apparent through the following example. The formula

$$
f_{\lambda}(x):=\frac{1}{1+\lambda^{2}\left(\sum_{j=1}^{d} x_{j}^{2}\right)} \quad \lambda \in \mathbb{R}
$$

gives a distribution for each fixed $\lambda$ and it is natural to say that it depends analytically on $\lambda$. Unfortunately, as follows from [40, p. 107] or [41, p. 97] there is no reasonable topology on the space of distributions (or smooth functions) such that $\lambda \mapsto f_{\lambda}$ is locally given by a convergent vector valued Taylor series.

The Acta Mathematica paper of Kriegl and Michor [40] (and also their subsequent book [41]) contains a detailed discussion on "real analytic dependence" in the context of their study of real analytic maps between manifolds modelled on locally convex spaces and respective vector bundles. Their research is motivated, for instance, by the search of a real analytic structure of the group of real analytic diffeomorphisms on a real analytic manifold. In order to cover examples like (1.1) we have to follow the choice of Kriegl and Michor: for $U$ a real analytic manifold and $E$ a complex locally convex space 
we call a vector valued function $g: U \rightarrow E$ real analytic if for every linear continuous functional $x \in E^{\prime}$ the scalar function $x \circ g: U \rightarrow \mathbb{C}$ is analytic. In the context of distributions our definition means that

$$
U \ni \lambda \mapsto f_{\lambda} \in \mathscr{D}^{\prime}(\Omega)
$$

is real analytic if for every test function $\varphi \in \mathscr{D}(\Omega)$ the scalar function

$$
\lambda \mapsto\left\langle f_{\lambda}, \varphi\right\rangle
$$

is real analytic. The example (1.1) satisfies that definition but it is not covered by Browder's approach because his analytic dependent objects must be locally represented by the vector valued Taylor series convergent in $L_{2}(\Omega)$, hence also in $\mathscr{D}^{\prime}(\Omega)$. Moreover, not every real analytic vector valued function extends to a holomorphic function on some complex neighbourhood of its domain (again the example (1.1)!) and therefore the real analytic dependence differs essentially from the holomorphic one. A characterization of Fréchet spaces $X$ such that all $X$-valued real analytic functions are locally representable via convergent vector valued Taylor series is given in [4] and [5] but neither the space of smooth functions on a manifold nor the space of distributions satisfies this condition.

The research of Kriegl and Michor in [40] and [41] gives the second motivation of our problem. In their terminology, we ask if every real analytic curve lifts with respect to a surjective linear partial differential operator - in [41, Sec. 21] such a lifting problem is considered for arbitrary quotient maps and the smooth analogue of the question is explicitly formulated as an open problem [41, p. 226].

The author finds the outcome of the present paper as very surprising. We know (see, for instance, discussion in [8]) that the analogous smooth or holomorphic dependence problems for $P(D): \mathscr{D}^{\prime}(\Omega) \rightarrow \mathscr{D}^{\prime}(\Omega)$ have always positive solutions over any convex open set $\Omega \subseteq \mathbb{R}^{d}$ and for any linear partial differential equation with constant coefficients. Contrary to that, we will prove:

Theorem A. The real analytic parameter dependence problem for a partial differential operator $P(D): \mathscr{D}^{\prime}(\Omega) \rightarrow \mathscr{D}^{\prime}(\Omega)$ with constant coefficients:

(a) has a negative answer for all elliptic operators for arbitrary open set $\Omega \subseteq \mathbb{R}^{d}, d>1$, or for hypoelliptic operators for arbitrary open convex set $\Omega \subseteq \mathbb{R}^{d}, d>1$ as well as for a system of Cauchy-Riemann equations for arbitrary pseudoconvex domain $\Omega \subseteq \mathbb{C}^{d}, d \geq 1$;

(b) has an affirmative answer for operators of two variables on convex open $\Omega \subseteq \mathbb{R}^{d}$ if and only if the operator is hyperbolic; 
(c) has an affirmative answer for operators of order two on $\mathbb{R}^{d}$ if and only if after some real linear invertible change of variables the operator $P(D)$ is of the form

$$
\mu\left(\partial_{1}-a_{1}\right)^{2}+c \quad \text { or } \quad \mu\left[\sum_{j=1}^{r}\left(\partial_{j}-a_{j}\right)^{2}-\sum_{j=r+1}^{s-1}\left(\partial_{j}-a_{j}\right)^{2}+\lambda i \partial_{s}\right]+c
$$

for $\mu, c, a_{j} \in \mathbb{C}, \lambda \in \mathbb{R}, 1 \leq r<s-1 ;$

(d) has an affirmative answer for operators with homogeneous symbol on convex open $\Omega \subseteq \mathbb{R}^{d}$ if and only if the operator has a linear continuous right inverse on $C^{\infty}(\Omega)$.

In fact, in all the cases above, the solution of our problem is positive if and only if $P(D): \mathscr{D}^{\prime}(\Omega) \rightarrow \mathscr{D}^{\prime}(\Omega)$ or, equivalently, $P(D): C^{\infty}(\Omega) \rightarrow C^{\infty}(\Omega)$ has a linear continuous right inverse.

As a consequence, the problem of real analytic parameter dependence of solutions has a negative answer for the Laplace, heat and Schrödinger operators on any open convex set $\Omega$ as well as for the wave operator with more than one spatial variable on any bounded convex set with $C^{1}$-boundary. On the other hand, the answer is affirmative for the wave operator with arbitrarily many variables for $\Omega=\mathbb{R}^{d}$ as well as for the wave operator with one spatial variable for any open convex set $\Omega \subseteq \mathbb{R}^{2}$. Clearly, if a linear partial differential operator $P(D): \mathscr{D}^{\prime}(\Omega) \rightarrow \mathscr{D}^{\prime}(\Omega)$ (or, equivalently, $\left.P(D): C^{\infty}(\Omega) \rightarrow C^{\infty}(\Omega)[48]\right)$ has a linear continuous right inverse then it has a real analytic parameter dependence. All the above results support a surprising conjecture that the converse holds as well (and give a partial solution to [6, Problem 9.5]). A similar theory for operators on spaces of ultradifferentiable functions of Roumieu type, in particular, on spaces of real analytic functions, will be developed in a forthcoming paper.

Various characterizations of the existence of a linear continuous right inverse for a linear partial differential operator with constant coefficients (solving a problem of L. Schwartz) were given by Meise, Taylor and Vogt [48]. Their conditions were better understood and expressed in many cases in terms of the symbol thanks to efforts of Braun, Meise, Taylor and Vogt (see $[48,52,53,12]$ and many further papers). For homogeneous symbols $P$ and bounded convex $\Omega$ with $C^{1}$-boundary this holds if and only if $P$ is proportional to a product of linear forms with real coefficients [48, Th. 3.8]. For $\Omega=\mathbb{R}^{d}$ the condition is well understood up to $d=3$ [12]. Our research both shed a new light on those results and profits from the deep ideas in the papers listed above (especially, from [55]) which are truly impressive and inspiring for us. 
We will prove also some unexpected relations, in particular, that operators with real analytic parameter dependence are just perturbations of homogeneous operators with the same property:

Theorem B. If a linear partial differential operator $P(D): \mathscr{D}^{\prime}(\Omega) \rightarrow \mathscr{D}^{\prime}(\Omega)$ with constant coefficients, $\Omega \subseteq \mathbb{R}^{d}$ convex open, has a real analytic parameter dependence then both $P(D): \mathscr{D}^{\prime}\left(\mathbb{R}^{d}\right) \rightarrow \mathscr{D}^{\prime}\left(\mathbb{R}^{d}\right)$ and $P_{p}(D): \mathscr{D}^{\prime}(\Omega) \rightarrow \mathscr{D}^{\prime}(\Omega)$ satisfy the same condition, where

$$
P_{p}(z)=\sum_{|\alpha|=k} a_{\alpha} z^{\alpha}
$$

is the principal part of the polynomial $P(z)=\sum_{|\alpha| \leq k} a_{\alpha} z^{\alpha}$.

By the Köthe-Grothendieck duality [43, Prop. 6.12], the dual space $H^{\prime}(\mathbb{D})$ of the space of holomorphic functions on the unit disc $H(\mathbb{D})=\operatorname{ker} \bar{\partial} \subseteq$ $C^{\infty}(\mathbb{D})$ can be identified as follows:

$$
\begin{gathered}
H^{\prime}(\mathbb{D})=\operatorname{ind}_{N \in \mathbb{N}}\left(H_{0}^{\infty}\left(K_{N}\right),\|\cdot\|_{N}^{*}\right), \\
K_{N}:=\mathbb{C}^{*} \backslash(1-1 / N) \overline{\mathbb{D}}, \quad\|f\|_{N}^{*}:=\sup _{|z|>1-1 / N}|f(z)|,
\end{gathered}
$$

where the subscript 0 means that we consider functions vanishing at $\infty$, $\mathbb{C}^{*}=\mathbb{C} \cup\{\infty\}$ the Riemann sphere. The Hadamard three circle theorem implies that

$$
\left.\forall N \exists M \forall K \exists \theta_{0} \forall \theta \in\right] \theta_{0}, 1\left[\exists C \quad\|f\|_{M}^{*} \leq C\left(\|f\|_{N}^{*}\right)^{\theta}\left(\|f\|_{K}^{*}\right)^{1-\theta} .\right.
$$

In fact, this condition is nothing more but a qualitative version of the Hadamard's theorem later generalized by Petsche [66, Cor. 4.5]. In [6], [7] and [8] the problem of splitting of short exact sequences

$$
0 \longrightarrow X \longrightarrow Y \longrightarrow F \longrightarrow 0
$$

was studied for $F$ either Fréchet or dual Fréchet and $X, Y$ the so-called PLS-spaces (like spaces of distributions or their closed subspaces, see Section 2). The conditions analogous to (1.2) but for non-metrizable spaces were discovered and proved to be of crucial importance for splitting. In particular, in [6, Cor. 8.4], [8, Prop. 5.4] it was shown that essentially the same condition as (1.2), the so-called dual interpolation estimate for $\theta$ close to 1 (see (2.1) below in Section 2) holds for kernels of arbitrary partial differential operators with constant coefficients (not only $\bar{\partial}$ ) on $\mathscr{D}^{\prime}(\Omega), \Omega \subseteq \mathbb{R}^{d}$ convex. The basis of the whole paper is the following surprising fact that the strong version of the Hadamard's estimate has dramatic consequences (Corollary 6.4): 
Theorem C. An arbitrary surjective linear partial differential operator $P(D, x)$ with variable coefficients on $\mathscr{D}^{\prime}(\Omega)$ has real analytic parameter dependence of solutions if and only if its kernel has the dual interpolation estimate for all sufficiently small $\theta \in] 0,1[$ (see (2.1)). In fact, this result holds for an arbitrary surjective linear continuous operator $T: \mathscr{D}^{\prime}(\Omega) \rightarrow \mathscr{D}^{\prime}(\Omega)$ as well, where $\mathscr{D}^{\prime}(\Omega)$ is either the space of distributions on open $\Omega \subseteq \mathbb{R}^{d}$ or the space of currents on a $C^{\infty}$-manifold $\Omega$.

It is worth noting that since $\mathscr{D}^{\prime}(\Omega)^{m} \simeq \mathscr{D}^{\prime}(\Omega)$ the result holds for matrices (systems) of differential operators as well.

The whole paper is just the proof of the results above. It is based on a combination of methods from various branches of mathematics and it also gives several interesting by-products. In fact, roughly half of the paper consists of estimates on plurisubharmonic functions on algebraic varieties. First, we use some functional analysis to reformulate the problem to the question on surjectivity of tensor products of surjective operators. The second step is based on homological algebra and gives a reformulation in terms of vanishing of some derived functor, or more precisely, some special cohomology groups $\operatorname{Proj}^{1}$. The third part based on the operator theory on Hilbert spaces (spectral theory of unbounded operators) and on the modern theory of locally convex spaces evaluates when $\operatorname{Proj}^{1}=0$. The fourth step comes from the function theory: it is a specification of the calculated condition for the space of real analytic functions via a complex analytic construction to obtain the mentioned above dual interpolation estimate for small $\theta$. The fifth part is a translation of the estimate for kernels of differential operators through the fundamental principle of Ehrenpreis and Palamodov. The sixth part, the longest one, is a mixture of algebraic geometry and the theory of plurisubharmonic functions. We obtain estimates for plurisubharmonic functions on the zero variety of the polynomial $P$ which give main results.

Let us emphasize that several results in the paper are formulated and proved for $\mathscr{D}_{(\omega)}^{\prime}(\Omega)$ (including Denjoy-Carleman style ultradistributions $\mathscr{D}_{\left(M_{p}\right)}^{\prime}$ -see [39]) but they cover also the most interesting case of the space of classical distributions $\mathscr{D}^{\prime}(\Omega)$ by taking $\omega(t)=\log (2+|t|)$. All the results up to Section 6 are valid also for operators with variable coefficients and $\Omega$ being a smooth manifold (that means for spaces of currents instead of distributions).

Now, we summarize the content of the paper. In Section 2 we explain the framework of the category of PLS-spaces which contains most of the nonBanach natural spaces of analysis. Among them the spaces of (ultra-)distributions, currents, real analytic functions, smooth and holomorphic functions as well as the spaces of tempered distributions. We recall topological invariants extensively used in the paper: dual interpolation estimates and the so-called $P$-conditions. 
In Section 3 we formulate precisely the problem of real analytic parameter dependence of solutions of the given distributional equation $T u=f$, where $T, f$ are given and $u$ is an unknown distribution or current. We recall that this is equivalent with surjectivity of the operator $T \otimes \mathrm{id} \mathscr{A}(U)$ defined on the completed tensor product of the domain of $T$ and the space of real analytic functions $\mathscr{A}(U)$. Grothendieck showed that for nuclear Fréchet spaces tensor product of two surjective operators is surjective as well but we consider highly non-metrizable spaces like $\mathscr{D}^{\prime}(\Omega)$ or $\mathscr{A}(U)$ and therefore our problem of surjectivity of tensor products is much more subtle and so far it has been unsolved - the results of the present paper (see Section 5) are the strongest known up to now.

In Section 4 we explain the relation of the problem of surjectivity of tensor products of surjective operators acting on PLS-spaces to the homological algebra problem of vanishing of $\operatorname{Proj}^{1}$ functor (the first derived functor of the functor of projective limit) on the tensor product of kernels, see Corollary 4.6. This section is the last part in the introductory part containing essentially known results with only minor original contribution.

Section 5 is the first technical core of the paper. The main result (Theorem 5.2) characterizes when $\operatorname{Proj}^{1}$ vanishes for a completed tensor product of two PLN-spaces. Via the results of Section 4 it solves completely the problem of surjectivity of tensor products of two surjective endomorphisms on PLN-spaces (therefore on most of the natural spaces of analysis)!

In Section 6 we draw consequences from the previous section for tensor products $X \hat{\otimes}_{\varepsilon} E$ when one space is fixed. The crucial result is Corollary 6.4 $=$ Theorem $\mathrm{C}$ which characterizes when $T \otimes \mathrm{id} \mathscr{A}(U)$ is surjective for surjective $T: \mathscr{D}^{\prime}(\Omega) \rightarrow \mathscr{D}^{\prime}(\Omega)$ or, equivalently, characterizes when the equation $T u=f$ has a real analytic parameter dependence of solutions. As a consequence we characterize in terms of $\mu$ when a convolution operator $T_{\mu}: \mathscr{D}_{(\omega)}^{\prime}(\mathbb{R}) \rightarrow$ $\mathscr{D}_{(\omega)}^{\prime}(\mathbb{R})$ has a real analytic parameter dependence of solutions. The section contains only a selection of the consequences of the constructed theory - an attentive reader will easily produce much more.

Since in Section 6 we show that surjectivity of tensorized maps/real analytic parameter dependence is equivalent with the so-called dual interpolation estimates for the kernel of the map $T$, it is of crucial importance to decide for a fixed operator $T$ (especially differential operator) when its kernel has this property. In Section 7 we give a characterization for linear partial differential operators with constant coefficients, see Theorem 7.1. We use the fundamental principle of Ehrenpreis and Palamodov and theory of plurisubharmonic functions to translate our conditions into a Phragmén-Lindelöf type estimate for plurisubharmonic functions on a suitable algebraic variety. 
In Section 8 we use our theory to the problem of real analytic parameter dependence of solutions of linear partial differential equations with constant coefficients on the space of distributions $\mathscr{D}^{\prime}(\Omega)$ or the space of ultradistributions of Beurling type $\mathscr{D}_{(\omega)}^{\prime}(\Omega), \Omega$ convex. Using plurisubharmonic functions on algebraic varieties we show the main results of the paper (Theorems A and B). This section is the second technical core of the paper - we apply subtle methods of the theory of plurisubharmonic functions.

The last Section 9 collects open problems which naturally appear in our theory.

To conclude the introductory section let us recall that Fréchet space means a complete metrizable locally convex space. An operator denotes a linear continuous map and by $L(X, Y)$ we denote the space of all such operators $T: X \rightarrow Y$. The notation $X \stackrel{\text { comp }}{\subseteq} Y$ means that $X$ is a complemented locally convex subspace of $Y$. By $\Omega$ we always denote either an open subset of $\mathbb{R}^{d}$ or a smooth manifold both with a fixed but arbitrary compact exhaustion

$$
K_{1} \Subset K_{2} \Subset \cdots \Subset K_{N} \Subset \cdots \Subset \Omega, \quad \Omega=\bigcup_{N \in \mathbb{N}} K_{N} .
$$

From Section 7 on, $\Omega$ is always an open subset of $\mathbb{R}^{d}$. If $\Omega$ is convex then $K_{N}$ will be chosen convex as well. By $\mathscr{A}(U)$ we denote the space of real analytic functions with its natural topology (see $[47,18,24]$ ) on a real analytic manifold $U$. Throughout the paper we always assume that real analytic manifolds are Hausdorff, paracompact and have countable bases of topology.

A short exact sequence of locally convex spaces

$$
0 \longrightarrow X \stackrel{j}{\longrightarrow} Y \stackrel{q}{\longrightarrow} Z \longrightarrow 0
$$

is called topologically exact if $j$ is a linear topological embedding and $q$ is an open continuous linear map. By $B(x, r)$ we denote a ball of radius $r$ and center $x . H(V)$ denotes the space of holomorphic functions on $V$. If $z=\left(z_{1}, \ldots, z_{d}\right) \in \mathbb{C}^{d}$ then $|z|:=\left(\sum_{j=1}^{d}\left|z_{j}\right|^{2}\right)^{1 / 2}$. The symbols $o(\cdot)$ and $O(\cdot)$ are always understood as variable tends to $\infty$.

For non-explained notions from functional analysis (theory of locally convex spaces, spectral theory etc.) see [57] or/and [37]. For the theory of tensor products see [37]. For applications of homological algebra and derived functors to partial differential equations and functional analysis see [83]. For the theory of plurisubharmonic functions (pluripotential theory) see [38]. For linear partial differential equations see $[36,62]$. For the theory of currents see [25]. 


\section{PLS-spaces - preliminaries}

The whole paper is written in a very convenient framework of the so-called PLS-spaces. It turns out that typical non-Banach spaces of analysis are either PLS-spaces or their duals. For a review on PLS-spaces see [18].

An $L S$-space (called also a DFS-space) is a locally convex inductive limit of a sequence of Banach spaces with compact linking maps or, equivalently, a strong dual of a Fréchet-Schwartz space. It is known that LS-spaces are reflexive, Schwartz, separable and that every closed subspace and separated quotient of an LS-space is LS as well. Examples of LS-spaces are spaces of distributions of compact support $\mathscr{E}^{\prime}(\Omega)$, spaces of tempered distributions $\mathscr{S}^{\prime}$, spaces of germs of holomorphic functions over a compact set $H(K)$, etc.

A projective limit of a sequence of LS-spaces is called a PLS-space. It is known that all Fréchet-Schwartz spaces, LS-spaces and their countable products are PLS-spaces. Every closed subspace and complete separated quotient of a PLS-space is PLS as well. The class of PLS-spaces is the smallest class of locally convex spaces containing Fréchet-Schwartz spaces, their duals and closed with respect of taking countable products and closed subspaces.

PLS-spaces are separable, webbed and complete. They are reflexive if and only if they are barrelled or (ultra)bornological. Examples of PLSspaces are spaces of holomorphic functions $H(V)$, smooth functions $C^{\infty}(\Omega)$, distributions or currents $\mathscr{D}^{\prime}(\Omega)$, real analytic functions $\mathscr{A}(U)$, ultradistributions in the sense of Beurling $\mathscr{D}_{(\omega)}^{\prime}(\Omega)$, ultradifferentiable functions in the sense of Roumieu $\mathscr{E}_{\{\omega\}}(\Omega)$ and Köthe type PLS-spaces $\Lambda(A)$ (see [18]).

If instead of LS-spaces we take LN-spaces (i.e., locally convex inductive limits of sequences of Banach spaces with nuclear linking maps, called also DFN-spaces since they are strong duals of nuclear Fréchet spaces), we get PLN-spaces. All the above mentioned PLS-spaces except some Köthe spaces $\Lambda(A)$ are PLN-spaces. The category of PLS-spaces is more convenient since not every nuclear Fréchet space is a PLN-space (only so-called strongly nuclear Fréchet spaces [37] are PLN-spaces).

If $X$ is a PLS-space we write it throughout the paper as

$$
X=\operatorname{proj}_{N \in \mathbb{N}} X_{N}, \quad \text { where } X_{N}=\operatorname{ind}_{n \in \mathbb{N}} X_{N, n} \text { is an LS-space, }
$$

$\left(X_{N, n},\|\cdot\|_{N, n}\right)$ are Banach spaces, $i_{N, n+1}^{n}: X_{N, n} \rightarrow X_{N, n+1}$ are compact linking maps, $i_{N}^{N+1}: X_{N+1} \rightarrow X_{N}, i_{N}: X \rightarrow X_{N}$ are linking maps. Moreover, we denote by $B_{N, n}$ the unit ball of $X_{N, n}$ and $U_{N, n}$ its polar, i.e., the unit ball in $X_{N, n}^{\prime} \hookrightarrow X_{N}^{\prime}$. Without loss of generality we assume that $i_{N, n+1}^{n}$ has norm $\leq 1$, i.e., $B_{N, n} \subseteq B_{N, n+1}$ for $N, n \in \mathbb{N}$. Moreover,

$$
i_{N}^{N+1}\left(B_{N+1, n}\right) \subseteq B_{N, n} \quad \text { for } N, n \in \mathbb{N} .
$$


We denote

$$
\|f\|_{N, n}^{*}:=\sup _{\|x\|_{N, n} \leq 1}|f(x)|
$$

the dual norm. The same conventions will be used for other PLS-spaces $Y, E$, etc. By $W\left(U_{N, n}, B_{N, n}\right)$ we denote the set of all linear continuous operators

$$
T: X_{N, n}^{\prime} \rightarrow Y_{N, n}, \quad T\left(U_{N, n}\right) \subseteq B_{N, n} .
$$

The spaces mentioned above are described, for instance, in [6, pp. 333-336]. For the sake of completeness we recall the definition of $\mathscr{D}_{(\omega)}^{\prime}(\Omega)$ from $[11]$ since these spaces are extensively used in the paper.

We will use the classical multiindex notation for $\alpha=\left(\alpha_{1}, \ldots, \alpha_{d}\right)$,

$$
|\alpha|=\alpha_{1}+\cdots+\alpha_{d}, \quad f^{(\alpha)}(x)=\frac{\partial^{|\alpha|}}{\partial^{\alpha_{1}} x_{1} \cdots \partial^{\alpha_{d}} x_{d}} .
$$

We introduce a weight $\omega:[0, \infty[\rightarrow[0, \infty[$ to be a continuous increasing function satisfying the following conditions:

$(\alpha) \omega(2 t)=O(\omega(t))$;

$(\beta) \omega(t)=O(t)$

$(\gamma) \log t=o(\omega(t))$ or $\omega(t)=\log (2+|t|)$;

$(\delta) \varphi$ is a convex function, $\varphi(t):=\omega\left(e^{t}\right)$.

We extend $\omega$ to $\mathbb{C}^{d}$ by $\omega(z):=\omega(|z|)$. If

$$
\int_{0}^{\infty} \frac{\omega(t)}{1+t^{2}} d t<\infty
$$

then the weight is non-quasianalytic and we will use later on only such weights.

If $\Omega \subseteq \mathbb{R}^{d}$ open then the class of Beurling ultradistributions $\mathscr{D}_{(\omega)}^{\prime}(\Omega)$ is defined to be the strong dual of

$$
\begin{aligned}
\mathscr{D}_{(\omega)}(\Omega):=\{f \in \mathscr{D}(\Omega): \forall k \in \mathbb{N} \\
\left.\|f\|_{k}:=\sup _{x \in \Omega} \sup _{\alpha \in \mathbb{N}^{d}}\left|f^{(\alpha)}(x)\right| \exp \left(-k \varphi^{*}\left(\frac{|\alpha|}{k}\right)\right)<\infty\right\},
\end{aligned}
$$

where

$$
\varphi^{*}(t):=\sup _{x \geq 0}(x t-\varphi(t)) \text { the Young conjugate of } \varphi,
$$

equipped with the inductive limit topology (steps are Fréchet spaces with $\|\cdot\|_{k}$ seminorms):

$$
\mathscr{D}_{(\omega)}(\Omega)=\operatorname{ind}_{N \in \mathbb{N}} \mathscr{D}_{(\omega)}\left(K_{N}\right), \quad \mathscr{D}_{(\omega)}\left(K_{N}\right):=\left\{f \in \mathscr{D}_{(\omega)}(\Omega): \operatorname{supp} f \subseteq K_{N}\right\} .
$$


Thus $\mathscr{D}_{(\omega)}^{\prime}(\Omega)$ is a PLN-space. We consider the weight $\omega(t)=\log (2+|t|)$ since it gives $\mathscr{D}_{(\omega)}^{\prime}(\Omega)$ to be the classical space of distributions $\mathscr{D}^{\prime}(\Omega)$. If $\Omega$ is a smooth manifold then we use the same notation $\mathscr{D}^{\prime}(\Omega)$ to denote the space of currents over $\Omega$ with the analogous topology (we hope that which space is denoted will be clear from the context).

The family of classes $\mathscr{D}_{(\omega)}^{\prime}$ is well adapted to Fourier transform but it contains also most of the Denjoy-Carleman style classes defined via growth conditions of Taylor coefficients controlled by sequences $\left(M_{p}\right)$, see [9] and [39]. The best known among them are Gevrey classes (see, for instance, [69]): $\Gamma_{(p)}=\mathscr{D}_{(\omega)}$ for $\omega(t)=t^{1 / p}$ for $p>1$.

The space of real analytic functions $\mathscr{A}(\Omega)$ has the topology given as

$$
\mathscr{A}(\Omega)=\operatorname{proj}_{N \in \mathbb{N}} H\left(K_{N}\right),
$$

where $H\left(K_{N}\right)$ is the space of germs of holomorphic functions over $K_{N}$,

$$
H\left(K_{N}\right):=\operatorname{ind}_{n \in \mathbb{N}} H^{\infty}\left(U_{N, n}\right),
$$

where $\left(U_{N, n}\right)_{n \in \mathbb{N}}$ is a basis of complex neighbourhoods of $K_{N}$. Analogously, we define topology on $\mathscr{A}(\Omega)$ where $\Omega$ is a real analytic manifold. Clearly $\mathscr{A}(\Omega)$ is a PLN-space (for more details see [18]). The space $\mathscr{A}(\Omega), \Omega \subseteq \mathbb{R}^{d}$ open, is neither metrizable nor dual metrizable and it has a complicated structure: for instance, it has no Schauder basis [24].

We call a sequence $\alpha$ regular if

$$
\Lambda_{r}(\beta) \stackrel{\text { comp }}{\subseteq} \Lambda_{r}(\alpha) \stackrel{\text { comp }}{\subseteq} \Lambda_{r}(\gamma), \quad \beta, \gamma \text { stable. }
$$

Here $\Lambda_{0}(\alpha), \Lambda_{\infty}(\alpha)$ denote power series spaces of finite and infinite type, respectively, see [57, Ch. 29].

We will need the following simple observation:

Proposition 2.1 Let $U$ be a real analytic manifold.

(a) If $U$ is compact then $\mathscr{A}(U) \simeq \Lambda_{0}^{\prime}(\alpha)$, $\alpha$ regular.

(b) If $U$ has infinitely many connected components, all of them compact, then

$\mathscr{A}(U) \simeq \prod_{n \in \mathbb{N}} \Lambda_{0}^{\prime}\left(\alpha^{(n)}\right), \quad$ sequences $\alpha^{(n)}$ are regular for every $n \in \mathbb{N}$.

(c) If $U$ has at least one non-compact connected component then $\mathscr{A}(U)$ has a quotient isomorphic to $\mathscr{A}(\mathbb{R})$ and $\mathscr{A}(U)$ is isomorphic to a quotient of the space $\mathscr{A}\left(\mathbb{R}^{m}\right)$ for suitable big $m$. 
Proof. (a): Clearly $U$ contains a copy of the unit circle $\mathbb{T}$ as a real analytic submanifold. By [81], the restriction map $r: \mathscr{A}(U) \rightarrow \mathscr{A}(\mathbb{T})$ has a continuous linear right inverse, so $\mathscr{A}(\mathbb{T}) \simeq \Lambda_{0}^{\prime}(j)$ is isomorphic to a complemented subspace of $\mathscr{A}(U)$. By [60, §2.15], we may embed $U$ into $\mathbb{T}^{m}$ for $m$ big enough. Again by [81], the restriction map $R: \mathscr{A}\left(\mathbb{T}^{m}\right) \rightarrow \mathscr{A}(U)$ has a continuous linear right inverse, so $\mathscr{A}(U)$ is complemented in $\mathscr{A}\left(\mathbb{T}^{m}\right) \simeq \Lambda_{0}^{\prime}\left(j^{1 / m}\right)$. This completes the proof by [59, Cor. 1.1], comp. [75, Satz 1.7].

(b): Immediate consequence of (a).

(c): Obviously, we may $C^{1}$-embed $\mathbb{R}$ into $U$. By [34, Th. 2.1.4, 2.5.1], we can also embed $\mathbb{R}$ into $U$ as a real analytic submanifold. The restriction map $r: \mathscr{A}(U) \rightarrow \mathscr{A}(\mathbb{R})$ is a linear continuous surjection. Analogously, by $[60, \S 2.15], U$ embeds into $\mathbb{R}^{m}$ for sufficiently big $m$ as a real analytic submanifold and the restriction map $R: \mathscr{A}\left(\mathbb{R}^{m}\right) \rightarrow \mathscr{A}(U)$ is a linear continuous surjection. By [47, Th. 1.2] and [57, 24.30] the maps $r$ and $R$ are open.

We will use extensively in the paper the following Lemma [11, Lemma 1.7], [10, Prop. 5], [50, Lemma 3.7]:

Lemma 2.2 For every non-quasianalytic weight $\omega, \varepsilon>0$, and a function $h:[0, \infty[\rightarrow[0, \infty[$ such that $\omega=o(h)$ there exists a non-quasianalytic weight $\sigma \geq \omega$, a plurisubharmonic function $v$ on $\mathbb{C}^{d}$ and a constant $D>0$ such that

$$
\omega=o(\sigma), \quad \sigma=o(h), \quad-D \omega(z) \leq v(z) \leq \varepsilon|\operatorname{Im} z|-\omega(z) \quad \text { for } z \in \mathbb{C}^{d} .
$$

We will use only PLS-type Köthe sequence spaces with $\ell_{\infty}$-norms as defined, for instance, in [6]. Among PLS-type Köthe sequence spaces the most interesting are PLS-type power series spaces $\Lambda_{r, s}(\alpha, \beta)$ see for instance [6, Th. 2.2]. We define for sequences $\left(\alpha_{j}\right)$ and $\left(\beta_{j}\right)$ of positive numbers such that $\alpha_{j}+\beta_{j} \rightarrow \infty$ as $j \rightarrow \infty$ the following PLS-space

$$
\Lambda_{r, s}(\alpha, \beta):=\left\{x=\left(x_{j}\right): \forall N \exists n:\|x\|_{N, n}<\infty\right\}
$$

where

$$
\|x\|_{N, n}:=\sup _{j}\left|x_{j}\right| \exp \left(r_{N} \alpha_{j}-s_{n} \beta_{j}\right)
$$

and $\left(r_{N}\right),\left(s_{n}\right)$ are arbitrary fixed sequences such that $r_{N} \nearrow r, s_{n} \nearrow s$. It suffices to consider $r, s=0, \infty$ and the space does not depend on the choice of $\left(r_{N}\right)$ and $\left(s_{n}\right)$.

We will classify PLS-spaces by means of the so-called dual interpolation estimates and P-properties which are generalizations of $(\Omega)$-type and dual (DN)-type properties introduced by Vogt and Zahariuta (see MSC 2000 number 46A63) and were defined for the first time in $[6,7,8]$. For Fréchet spaces of holomorphic functions of one variable the estimates are closely connected (via Köthe-Grothendieck duality) with the classical Hadamard three 
circle theorem and operator theory specialists will recognize similarity to the so-called J-type in the interpolation theory of operators [2]. All these reminiscences are rooted in the proofs later on. A lot of information below is taken from [7]. A PLS-space is strict if and only if it is a projective limit of a sequence of LS-spaces where linking maps in the projective spectrum are surjective.

A PLS-space $X$ has a dual interpolation estimate if

$$
\begin{aligned}
& \forall N \exists M \geq N \forall K \geq M \exists n \forall m \geq n \forall \theta \in] 0,1[\exists k \geq m, C \\
& \forall x \in X_{N}^{\prime} \quad\left\|x \circ i_{N}^{M}\right\|_{M, m}^{*} \leq C\left(\left\|x \circ i_{N}^{K}\right\|_{K, k}^{*}\right)^{1-\theta}\left(\|x\|_{N, n}^{*}\right)^{\theta} .
\end{aligned}
$$

If we replace the quantifier $\forall \theta \in] 0,1[$ by

$$
\left.\exists \theta_{0} \in\right] 0,1[\forall \theta \in] 0, \theta_{0}\left[\quad\left(\text { or } \exists \theta_{0} \in\right] 0,1[\forall \theta \in] \theta_{0}, 1[\text { ) }\right.
$$

we get the definition of the dual interpolation estimate for small (big) theta.

Using the proof of $[6$, Lemma 5.1] we get the following reformulation of the dual interpolation estimate:

$$
\begin{aligned}
& \forall N \exists M \geq N \forall K \geq M \exists n \forall m \geq n \forall \theta \in] 0,1[\exists k \geq m, C \forall r>0 \\
& \quad \forall x \in X_{N}^{\prime}:\left\|x \circ i_{N}^{M}\right\|_{M, m}^{*} \leq C\left(r^{\theta}\left\|x \circ i_{N}^{K}\right\|_{K, k}^{*}+\frac{1}{r^{1-\theta}}\|x\|_{N, n}^{*}\right) .
\end{aligned}
$$

Clearly taking $\theta$ close to 0 or 1 we get the dual interpolation estimate for small or big $\theta$. If we consider only $r \geq 1$ then we get the condition $(P \overline{\bar{\Omega}})$ (for all $\theta$ or, equivalently, $\theta$ close to 0 ) or $(P \Omega$ ) (for $\theta$ close to 1 or, equivalently, for some $\theta \in] 0,1[)$. Analogously, if we consider only $0<r \leq 1$ then we get the condition $(P A)$ (for all $\theta$ or, equivalently, $\theta$ close to 1 ) or $(P \underline{A}$ ) (for $\theta$ close to 0 or, equivalently, for some $\theta \in] 0,1[$ ).

The dual interpolation estimate for small $\theta$ is equivalent to the combination of $(P \overline{\bar{\Omega}})$ and $(P \underline{A})$, for big $\theta$ is equivalent to $(P \Omega)$ and $(P A)$. Moreover, $(P \overline{\bar{\Omega}})$ implies $(P \Omega)$ and $(P A)$ implies $(P \underline{A})$. Thus the dual interpolation estimate for all $\theta$ is equivalent to the combination of $(P \overline{\bar{\Omega}})$ and $(P A)$.

There are also reformulations of these $\mathrm{P}$-conditions in the similar spirit as in the definition of the dual interpolation estimate. For instance, $(P \Omega)$ is equivalent to

$$
\begin{aligned}
& \forall N \exists M \geq N \forall K \geq M \exists n \forall m \geq n \exists \theta \in] 0,1[\exists k \geq m, C \\
& \forall x \in X_{N}^{\prime}:\left\|x \circ i_{N}^{M}\right\|_{M, m}^{*} \leq C\left(\left\|x \circ i_{N}^{K}\right\|_{K, k}^{*}\right)^{1-\theta} \cdot \max \left(\|x\|_{N, n}^{*},\left\|x \circ i_{N}^{K}\right\|_{K, k}^{*}\right)^{\theta} .
\end{aligned}
$$

If we take $\forall \theta \in] 0,1[$ then we get $(P \overline{\bar{\Omega}})$. Analogously, $(P \underline{A})$ is equivalent to

$$
\begin{aligned}
& \forall N \exists M \geq N \forall K \geq M \exists n \forall m \geq n \exists \theta \in] 0,1[\exists k \geq m, C \\
& \forall x \in X_{N}^{\prime}:\left\|x \circ i_{N}^{M}\right\|_{M, m}^{*} \leq C \max \left(\left\|x \circ i_{N}^{K}\right\|_{K, k}^{*},\|x\|_{N, n}^{*}\right)^{1-\theta}\left(\|x\|_{N, n}^{*}\right)^{\theta}
\end{aligned}
$$

and if we take $\forall \theta \in] 0,1[$ we get $(P A)$. 
All these conditions are inherited by countable products and complete quotients. In papers $[6,8,7]$ many examples of spaces with P-properties are collected. We summarize them for the sake of convenience (use Prop. 2.1).

Corollary 2.3 (a) A Fréchet space has a dual interpolation estimate for big $\theta$ iff it has $(\Omega)$. It has a dual interpolation estimate for small (all) $\theta$ iff it has $(\overline{\bar{\Omega}})$.

(b) An LS-space has a dual interpolation estimate for small $\theta$ iff its dual has $(\underline{D N})$. It has a dual interpolation estimate for big (all) $\theta$ iff its dual has $(D N)$.

(c) The space of distributions $\mathscr{D}^{\prime}(\Omega), \Omega \subseteq \mathbb{R}^{d}$ arbitrary open, or the space of currents $\mathscr{D}^{\prime}(\Omega), \Omega$ an arbitrary $C^{\infty}$-manifold, or the space of Beurling ultradistributions $\mathscr{D}_{(\omega)}^{\prime}(\Omega)$ has the dual interpolation estimate for all $\theta$.

(d) The space of real analytic functions $\mathscr{A}(\Omega), \Omega$ a real analytic manifold has the dual interpolation estimate for small $\theta$.

(e) The PLS-type power series space $\Lambda_{r, s}(\alpha, \beta)$ has the dual interpolation estimate for big $\theta$ iff $s=\infty$ or it is a Fréchet space. It has the dual interpolation estimate for small $\theta$ (or $(P \overline{\bar{\Omega}})$ ) iff it is an LS-space.

Remark. Let us recall that the space of currents $\mathscr{D}^{\prime}(\Omega)$ is the dual space of the space of differential forms on $\Omega$ with compact support. Since $\Omega$ can be embedded into some $\mathbb{R}^{m}$ in such a way that there is a tubular neighbourhood $\Omega_{1}$ of $\Omega$ in $\mathbb{R}^{m}$ and using $C^{\infty}$-resolution of identity we can define a linear continuous map extending differential forms with compact support on $\Omega$ to such forms on $\Omega_{1}$. By duality the space of currents $\mathscr{D}^{\prime}(\Omega)$ is a complemented subspace of the space of currents $\mathscr{D}^{\prime}\left(\Omega_{1}\right)$. Since $\Omega_{1}$ is "flat" the latter is a product of finitely many spaces of distributions. That is why the space of currents also has the dual interpolation estimate for all $\theta$.

\section{Parameter dependence of solutions versus surjectiv- ity of tensorized maps}

Let us assume that

$$
T: \mathscr{D}_{(\omega)}^{\prime}(\Omega) \rightarrow \mathscr{D}_{(\omega)}^{\prime}(\Omega)
$$

is a surjective operator and $U$ is a real analytic manifold. We say that the equation

$$
T u=f,
$$

where $u$ is an unknown distribution has the real analytic parameter dependence (of solutions) if for every real analytic function

$$
U \ni \lambda \mapsto f_{\lambda} \in \mathscr{D}_{(\omega)}^{\prime}(\Omega)
$$


there is a real analytic function

$$
U \ni \lambda \mapsto u_{\lambda} \in \mathscr{D}_{(\omega)}^{\prime}(\Omega)
$$

such that

$$
T u_{\lambda}=f_{\lambda} \quad \text { for every } \lambda \in U \text {. }
$$

Recall that for complete locally convex space $X$ :

$$
\mathscr{A}(U, X):=\left\{f: U \rightarrow X: \quad \forall g \in X^{\prime} \quad g \circ f \in \mathscr{A}(U)\right\} .
$$

As shown in [40], $\mathscr{A}(U, X)$ contains functions for which vector valued Taylor series is not convergent. In $[4,5]$ a characterization is given for which Fréchet spaces $X$ every function $F \in \mathscr{A}(U, X)$ has a Taylor series local representation.

Let us define $\varepsilon$-product of Schwartz for complete $X, E$ :

$$
X \varepsilon E:=L_{e}\left(X_{c o}^{\prime}, E\right)
$$

the space of linear continuous operators from the dual $X^{\prime}$ equipped with the compact-open topology. The subscript $e$ means that we equip $L\left(X_{c o}^{\prime}, E\right)$ with the topology of uniform convergence on equicontinuous sets. It is important that $\varepsilon$-product is commutative. Clearly $X \otimes E \subseteq X \varepsilon E$ and if one of the spaces has the approximation property then $X \otimes E$ is dense in $X \varepsilon E$, the latter is always complete if $E$ and $X$ are complete. If one of the spaces is nuclear (thus has the approximation property) then on $X \otimes E$ there is only one natural topology so then $X \varepsilon E$ is the unique natural completion of $X \otimes E$ !

It is well known that for complete $X$ (for $\mathscr{A}$ see [4, Th. 16]) spaces of vector valued functions have the following tensor representations:

$$
C^{\infty}(U, X)=C^{\infty}(U) \varepsilon X, \quad H(U, X)=H(U) \varepsilon X, \quad \mathscr{A}(U, X)=\mathscr{A}(U) \varepsilon X .
$$

For vector valued distributions $\varepsilon$-product is just the definition [71]:

$$
\mathscr{D}^{\prime}(U, X):=\mathscr{D}^{\prime}(U) \varepsilon X, \quad \mathscr{D}_{(\omega)}^{\prime}(U, X):=\mathscr{D}_{(\omega)}^{\prime}(U) \varepsilon X .
$$

As $T \otimes$ id $\mathscr{A}(U)$ we define the unique extension of the tensor map

$$
T \otimes \text { id } \mathscr{A}(U): \mathscr{D}_{(\omega)}^{\prime}(\Omega) \otimes \mathscr{A}(U) \rightarrow \mathscr{D}_{(\omega)}^{\prime}(\Omega) \otimes \mathscr{A}(U)
$$

via density to the map: $\mathscr{D}_{(\omega)}^{\prime}(\Omega) \varepsilon \mathscr{A}(U) \rightarrow \mathscr{D}_{(\omega)}^{\prime}(\Omega) \varepsilon \mathscr{A}(U)$.

Conclusion: Let $T: \mathscr{D}_{(\omega)}^{\prime}(\Omega) \rightarrow \mathscr{D}_{(\omega)}^{\prime}(\Omega)$ be an operator. The equation $T u=f$ has the real analytic parameter dependence of solutions if and only if

$$
T \otimes \text { id }: \mathscr{D}_{(\omega)}^{\prime}(\Omega) \varepsilon \mathscr{A}(U) \rightarrow \mathscr{D}_{(\omega)}^{\prime}(\Omega) \varepsilon \mathscr{A}(U)
$$

is surjective. An analogous conclusion holds for smooth, holomorphic, ultradifferentiable, (ultra)distributional dependence for operators on spaces of real analytic, smooth, ultradifferentiable, holomorphic functions or (ultra)distributions. 


\section{Surjectivity of tensorized maps versus $\operatorname{Proj}^{1}$ functor}

The aim of this section is to use homological approach to the problem of surjectivity of tensorized maps. We will translate the problem into the question of vanishing of the so-called Proj $^{1}$ functor. Of course, the main idea of this section is known to specialists (see [83]) - we complete it with some minor new results.

Let $V=\operatorname{proj}_{N \in \mathbb{N}} V_{N}$ be a projective limit of linear spaces. We construct the following exact sequence

$$
0 \longrightarrow V \stackrel{i}{\longrightarrow} \prod_{N \in \mathbb{N}} V_{N} \stackrel{\sigma}{\longrightarrow} \prod_{N \in \mathbb{N}} V_{N},
$$

where $i$ is the canonical embedding and $\sigma\left(\left(x_{n}\right)_{N \in \mathbb{N}}\right):=\left(i_{N}^{N+1} x_{N+1}-x_{N}\right)_{N \in \mathbb{N}}$ and $i_{N}^{N+1}: V_{N+1} \rightarrow V_{N}$ as usual denote linking maps. We define

$$
\operatorname{Proj}^{1}\left(\left(V_{N}, i_{N}^{N+1}\right)_{N \in \mathbb{N}}\right):=\prod_{N \in \mathbb{N}} V_{N} / \operatorname{im} \sigma .
$$

That functor was introduced to functional analysis by Palamodov [63, 64], and analyzed in depth by Vogt [78, 79], Frerick [28], Frerick and Wengenroth [30] and others, see a survey in [83].

If we assume that $\left(V_{N}\right)$ are complete and the spectrum is strongly reduced (i.e., canonical images of $V$ in $V_{N}$ are dense) then all such spectra are equivalent and we can write $\operatorname{Proj}^{1} V$ since it does not depend on such a spectrum representing $V$. If $V$ is a PLS-space then it is a strongly reduced projective limit of LS-spaces and we have the following result:

Theorem 4.1 [83, Cor. 3.3.10] Let $V$ be a PLS-space. The following assertions are equivalent:

1. $\operatorname{Proj}^{1} V=0$;

2. $V$ is ultrabornological;

3. $V$ is barrelled.

Moreover, the following essentially known result will be used, see [20, Prop. 4.3] and its proof based on [3, 4.3], where the last statement follows from [17, Prop. 2.7].

Theorem 4.2 Let $V=\operatorname{proj}_{N \in \mathbb{N}} V_{N}, W=\operatorname{proj}_{N \in \mathbb{N}} W_{N}$ are PLS-spaces (represented by strongly reduced spectra of LS-spaces) then

$$
V \varepsilon W=\operatorname{proj}_{N \in \mathbb{N}} V_{N} \varepsilon W_{N}=\operatorname{proj}_{N \in \mathbb{N}} L_{b}\left(V_{N}^{\prime}, W_{N}\right)
$$

is a PLS-space, spectrum $\left(V_{N} \varepsilon W_{N}\right)$ is strongly reduced and

$$
V_{N} \varepsilon W_{N}=L_{b}\left(V_{N}^{\prime}, W_{N}\right)
$$


is an LS-space with the fundamental sequence of bounded sets (Banach discs)

$$
\left(W\left(U_{N, n}, B_{N, n}\right)\right)_{n \in \mathbb{N}},
$$

where $B_{N, n}$ are the unit balls of Banach spaces in $W_{N, n}, W_{N}=\operatorname{ind}_{n \in \mathbb{N}} W_{N, n}$ and $U_{N, n}$ are the unit balls of the duals of $V_{N, n}, V_{N}=\operatorname{ind}_{n \in \mathbb{N}} V_{N, n}$.

We reduce the problem to tensorizing with the identity map.

Proposition 4.3 Let $S: X \rightarrow Y, T: E \rightarrow E$ be continuous surjective operators. Then

$$
S \otimes T: X \varepsilon E \rightarrow Y \varepsilon E
$$

is surjective if and only if

$$
\text { id }_{Y} \otimes T: Y \varepsilon E \rightarrow Y \varepsilon E, \quad \text { and } \quad S \otimes \text { id }_{E}: X \varepsilon E \rightarrow Y \varepsilon E
$$

are surjective.

Proof. Observe that

$$
S \otimes T=\left(\text { id }_{Y} \otimes T\right) \circ\left(S \otimes \text { id }_{E}\right)=\left(S \otimes \text { id }_{E}\right) \circ\left(\text { id }_{X} \otimes T\right) .
$$

Lemma 4.4 If $X$ is a nuclear Fréchet space then $X=\operatorname{proj}_{N \in \mathbb{N}} X_{N}$ such that $X_{N}$ are $L S$-spaces with $c_{0}$-steps. Therefore $X_{N}^{\prime}$ are projective limits of sequences of $\ell_{1}$ Banach spaces.

Proof. Clearly, by nuclearity, $X=\operatorname{proj}_{N \in \mathbb{N}} Y_{N}$, where $Y_{N} \simeq c_{0}$ and linking maps are compact.

If $T: c_{0} \rightarrow c_{0}$ is a compact operator then there is a null sequence $\left(x_{n}\right)$ such that

$$
T\left(B_{c_{0}}\right) \subseteq\left\{\left(z_{n}\right):\left|z_{n}\right|<x_{n}\right\} .
$$

Therefore $T$ factorizes through an LS-space (an inductive limit of $c_{0}$-Banach spaces):

$$
V:=\left\{\left(z_{n}\right): \exists N \sup _{n \in \mathbb{N}} \frac{\left|z_{n}\right|}{\sqrt[N]{x_{n}}}<\infty\right\}
$$

Thus every linking map $i_{N}^{N+1}: Y_{N+1} \rightarrow Y_{N}$ factorizes through an LS-space of type $V$.

Proposition 4.5 Assume that

$$
0 \longrightarrow X \longrightarrow Y \longrightarrow O
$$

is a topologically exact sequence of PLS-spaces, E a PLS-space. 
If either all $X_{N}^{\prime}$ or all $E_{N}^{\prime}$ are projective limits of Banach $\ell_{1}$-spaces (in particular, if either $X$ or $E$ is a PLN-space or a nuclear Fréchet space) then

$$
0 \longrightarrow X \varepsilon E \longrightarrow Y \varepsilon E \longrightarrow Z \varepsilon E \longrightarrow 0
$$

is exact if $\operatorname{Proj}^{1}{ }_{N \in \mathbb{N}}(X \varepsilon E)=0$. If $\operatorname{Proj}^{1}{ }_{N \in \mathbb{N}}(Y \varepsilon E)=0$ then the above condition is also necessary.

Proof. It is known (see [22, p. 64]) that (4.1) is a projective limit of exact sequences

$$
0 \longrightarrow X_{N} \stackrel{j_{N}}{\longrightarrow} Y_{N} \stackrel{q_{N}}{\longrightarrow} Z_{N} \longrightarrow 0
$$

where $Y=\operatorname{proj}_{N \in \mathbb{N}} Y_{N}, Z=\operatorname{proj}_{N \in \mathbb{N}} Z_{N}$ and $Y_{N}, Z_{N}$ are LS-spaces. By [37, proof of 16.3.2] (comp. Theorem 4.2),

$$
\begin{gathered}
X \varepsilon E=\operatorname{proj}_{N \in \mathbb{N}} X_{N} \varepsilon E_{N}, \quad Y \varepsilon E=\operatorname{proj}_{N \in \mathbb{N}} Y_{N} \varepsilon E_{N}, \\
Z \varepsilon E=\operatorname{proj}_{N \in \mathbb{N}} Z_{N} \varepsilon E_{N} .
\end{gathered}
$$

Since $E_{N}$ are LS-spaces, i.e., Montel thus $X_{N} \varepsilon E_{N}=L_{b}\left(E_{N}^{\prime}, X_{N}\right)$ etc. Therefore the sequence

$$
0 \longrightarrow X_{N} \varepsilon E_{N} \stackrel{j_{N} \otimes \mathrm{id}}{\longrightarrow} Y_{N} \varepsilon E_{N} \stackrel{q_{N} \otimes \mathrm{id}}{\longrightarrow} Z_{N} \varepsilon E_{N} \longrightarrow 0
$$

is exact if every $T \in L\left(E_{N}^{\prime}, Z_{N}\right)$ lifts with respect to $q_{N}$ to $S \in L\left(E_{N}^{\prime}, Y_{N}\right)$. By [6, Lemma 3.5] this is true (for the case of nuclear Fréchet spaces apply Lemma 4.4).

We have proved that (4.2) is a projective limit of exact sequences (4.3). The result follows from Palamodov's [63] or [83, Cor. 3.1.5] which implies existence of the following exact sequence:

$$
\begin{aligned}
0 \rightarrow X \varepsilon E & \rightarrow Y \varepsilon E \rightarrow Z \varepsilon E \rightarrow \\
& \rightarrow \operatorname{Proj}^{1}(X \varepsilon E) \rightarrow \operatorname{Proj}^{1}(Y \varepsilon E) \rightarrow \operatorname{Proj}^{1}(Z \varepsilon E) \rightarrow 0 .
\end{aligned}
$$

Corollary 4.6 Let $S: X \rightarrow Y, T: E \rightarrow E$ be continuous open surjective operators between PLS-spaces. Assume that either $E$ or $\operatorname{ker} T$ and $\operatorname{ker} S$ are PLN-spaces or nuclear Fréchet spaces. Then

$$
S \otimes T: X \varepsilon E \rightarrow Y \varepsilon E
$$

is surjective whenever

$$
\operatorname{Proj}^{1}(\operatorname{ker} S \varepsilon E)=\operatorname{Proj}^{1}(Y \varepsilon \text { ker } T)=0 .
$$

If, additionally, $\operatorname{Proj}^{1}(X \varepsilon E)=\operatorname{Proj}^{1}(Y \varepsilon E)=0$ then this is also a necessary condition.

Proof. Apply Proposition 4.5 and Proposition 4.3. 
Observe that:

Proposition 4.7 If $T: X \rightarrow Y$ is a surjective continuous operator between $P L S$-spaces and $\operatorname{Proj}^{1} Y=0$ then $T$ is open.

Proof. By Theorem 4.1, $Y$ is ultrabornological. Since any PLS-space $X$ is webbed, then by the De Wilde webbed open mapping theorem [57, 24.30], $T$ is open.

Finally, let us observe that vanishing of $\operatorname{Proj}^{1}$ is somehow inherited by products.

Proposition 4.8 Let $X, E^{(j)}, j \in \mathbb{N}$, be PLS-spaces. If $\operatorname{Proj}^{1}\left(X \varepsilon E^{(j)}\right)=0$ for every $j \in \mathbb{N}$ then $\operatorname{Proj}^{1}\left(X \varepsilon \prod_{j \in \mathbb{N}} E^{(j)}\right)=0$.

Proof. This is an easy calculation since $X \varepsilon \prod_{j \in \mathbb{N}} E^{(j)}=\prod_{j \in \mathbb{N}}\left(X \varepsilon E^{(j)}\right)$ and if $X=\operatorname{proj}_{N \in \mathbb{N}} X_{N}, E^{(j)}=\operatorname{proj}_{N \in \mathbb{N}} E_{N}^{(j)}$. Then

$$
\begin{aligned}
& \operatorname{Proj}^{1}\left(X \varepsilon \prod_{j \in \mathbb{N}} E^{(j)}\right)=\prod_{N \in \mathbb{N}}\left(X_{N} \varepsilon\left(\prod_{j \leq N} E_{N}^{(j)}\right)\right) / \operatorname{im} \sigma, \\
& \sigma: \prod_{N \in \mathbb{N}}\left(X_{N} \varepsilon\left(\prod_{j \leq N} E_{N}^{(j)}\right)\right) \rightarrow \prod_{N \in \mathbb{N}}\left(X_{N} \varepsilon\left(\prod_{j \leq N} E_{N}^{(j)}\right)\right) .
\end{aligned}
$$

Conclusion: To decide if the $\varepsilon$-product of two surjective operators between PLN-spaces is surjective we need to know when $\operatorname{Proj}^{1}$ of an $\varepsilon$-product of two PLN-spaces vanishes. We solve this problem in the next section.

\section{Vanishing of $\operatorname{Proj}^{1}$ for tensor products of PLS-spaces}

Now, we will give criteria of vanishing of $\operatorname{Proj}^{1}(X \varepsilon E)$, where $X, E$ are PLS-spaces. By Theorem 4.2, $X \varepsilon E$ is a PLS-space thus vanishing of $\mathrm{Proj}^{1}$ is inherited by complete quotients (use Theorem 4.1). Since $X$ and $E$ are complemented subspaces of $X \varepsilon E$ a necessary condition for $\operatorname{Proj}^{1} X \varepsilon E=0$ is that $\operatorname{Proj}^{1} X=\operatorname{Proj}^{1} E=0$. Moreover, there is a very good condition $\left(P_{3}^{*}\right)$ characterizing the vanishing of $\operatorname{Proj}^{1} V$ for PLS-spaces $V$ in terms of inequalities between norms on functionals on $V_{N}$ for a strongly reduced representation $V=\operatorname{proj}_{N \in \mathbb{N}} V_{N}$ (see [83, Cor. 3.3.10]). Unfortunately, for our purposes this is by far not enough since the space of functionals on $V_{N}=X_{N} \varepsilon E_{N}$ is too big to be handled and this condition gives no chance to "separate" the condition into suitable conditions on $X$ and $E$. 
A PLS space $E$ is called deeply reduced if one (equivalently, each one) of its strongly reduced representing spectra of LS-spaces $\left(E_{N}, i_{N}^{N+1}\right)$ satisfies the following condition

$$
\begin{array}{r}
\forall N \exists M \geq N \forall K \geq M \exists n \forall m \geq n \exists k \geq m: \\
i_{N}^{M} E_{M, m} \subseteq{\overline{i_{N}^{K} E_{K, k}}}^{E_{N, k}} \cap{\overline{E_{N, n}}}^{E_{N, k}} .
\end{array}
$$

If the inclusion holds only in the first (second) factor we call $E$ deeply reduced in columns (in rows).

Proposition 5.1 If $E$ is a deeply reduced in rows PLS-space then there is a strongly reduced representing spectrum $\left(\tilde{E}_{N}, i_{N}^{N+1}\right)$ such that

$$
\forall N \exists n \forall m \geq n \quad \tilde{E}_{N, m} \quad \text { is dense in } \quad \tilde{E}_{N, m+1} .
$$

If, additionally, E is just deeply reduced then we may additionally assume that

$$
\forall N \exists n \forall m \geq n \quad i_{N}^{N+1} \tilde{E}_{N+1, m} \quad \text { is dense } \tilde{E}_{N, m} .
$$

Remark. The converse implications are trivial.

Examples. The space of real analytic functions $\mathscr{A}(\Omega)$ is deeply reduced since every compact subset of $\mathbb{R}^{d}$ has a complex neighbourhood basis consisting of pseudoconvex sets (see [14] or [31]). It follows also from [70, Satz $2.19]$ that $\mathscr{E}_{\{\omega\}}(\Omega)$ is deeply reduced for quasianalytic weights $\omega$. Since for non-quasianalytic weights $\omega$ both $\mathscr{D}_{(\omega)}^{\prime}(\Omega)$ and $\mathscr{E}_{\{\omega\}}(\Omega)$ are Köthe type PLSspaces [76], [11], thus they are deeply reduced as all Köthe type PLS-spaces. It is also easy to show that every Fréchet Schwartz space is a deeply reduced PLS-space. On the other hand an LS-space is deeply reduced if and only if it is the dual of a countably normed Fréchet Schwartz space.

Proof of Proposition 5.1: Without loss of generality we may assume that $M=N+1$. First, let us assume only deep reducedness in rows. Thus for $m \geq n$ we define

$$
\tilde{E}_{N, m}:={\overline{E_{N, n}}}^{E_{N, m}} \subseteq E_{N, m}, \quad \tilde{E}_{N}:=\text { ind }{ }_{m \geq n} \tilde{E}_{N, m} .
$$

Since

$$
\forall N \exists n \forall m \geq n \exists k \geq m \quad i_{N}^{N+1} E_{N+1, m} \subseteq \tilde{E}_{N, k}
$$

the spectra $\left(E_{N}\right)$ and $\left(\tilde{E}_{N}\right)$ of LS-spaces are equivalent. Clearly, for $m \geq n$ the space $\tilde{E}_{N, m}$ is dense in $\tilde{E}_{N, m+1}$. 
Now, assume that $E$ is deeply reduced (also in columns!). By the above argument we may assume without loss of generality that for $m \geq n$ the space $E_{N, m}$ is dense in $E_{N, m+1}$ and that $k=m+1$. Thus

$$
\begin{aligned}
\forall N \exists & n(N) \forall m \geq n(N) \\
& i_{N}^{N+1} E_{N+1, m} \subseteq{\overline{i_{N}^{N+2} E_{N+2, m+1}}}^{E_{N, m+1}}=: F_{N, m+1} \subseteq E_{N, m+1} .
\end{aligned}
$$

We define $F_{N}:=$ ind ${ }_{m \geq n} F_{N, m}$ and it is an LS-space such that the spectrum $\left(F_{N}\right)$ is equivalent to $\left(E_{N}\right)$. Since for $m \geq n(N+1)$ holds

$$
i_{N}^{N+1} F_{N+1, m+1} \supseteq i_{N}^{N+1} i_{N+1}^{N+2} E_{N+2, m}
$$

we have

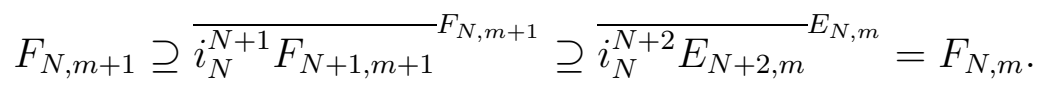

Since for $m$ big enough $E_{N+2, m}$ is dense in $E_{N+2, m+1}$ thus $i_{N}^{N+2} E_{N+2, m}$ is dense in $i_{N}^{N+2} E_{N+2, m+1}$ with respect to the topology of $E_{N, m+1}$. This means that $F_{N, m}$ is dense in $F_{N, m+1}$. By (5.1), also $i_{N}^{N+1} F_{N+1, m+1}$ is dense in $F_{N, m+1}$.

We are ready to formulate the main result of this section.

Theorem 5.2 Let $X, E$ be PLS-spaces which are strongly reduced projective limits of LS-spaces:

$$
\begin{aligned}
X & =\operatorname{proj}_{N \in \mathbb{N}} X_{N}=\operatorname{proj}_{N \in \mathbb{N}} \operatorname{ind}_{n \in \mathbb{N}} X_{N, n}, \\
E & =\operatorname{proj}_{N \in \mathbb{N}} E_{N}=\operatorname{proj}_{N \in \mathbb{N}} \operatorname{ind}_{n \in \mathbb{N}} E_{N, n} .
\end{aligned}
$$

If one of the following conditions hold:

(a) one of the spaces $X$ or $E$ is a deeply reduced PLN-space,

(b) both $X$ and $E$ are PLN-spaces,

(c) one of the spaces $X$ or $E$ is isomorphic to a Köthe type PLS-space of infinite order $\Lambda^{\infty}(A)$,

then the following conditions are equivalent:

(1) $\operatorname{Proj}^{1}(X \varepsilon E)=0$;

(2) the pair $(X, E)$ satisfies the condition $(T)$, i.e.,

$$
\begin{gathered}
\forall N \exists M \geq N \forall K \geq M \exists n \forall m \geq n \begin{aligned}
& \exists \forall m, S \\
& \forall x \in X_{N}^{\prime}, y \in E_{N}^{\prime}: \\
&\left\|x \circ i_{N}^{M}\right\|_{M, m}^{*}\left\|y \circ i_{N}^{M}\right\|_{M, m}^{*} \leq S\left(\|x\|_{N, n}^{*}\|y\|_{N, n}^{*}+\left\|x \circ i_{N}^{K}\right\|_{K, k}^{*}\left\|y \circ i_{N}^{K}\right\|_{K, k}^{*}\right) .
\end{aligned}
\end{gathered}
$$


(3) the pair $(X, E)$ satisfies the condition $\left(T_{\varepsilon}\right)$, i.e.,

$$
\begin{gathered}
\forall N \exists M \geq N \forall K \geq M \exists n \forall m \geq n, \varepsilon>0 \quad \exists k \geq m, S \\
\forall x \in X_{N}^{\prime}, y \in E_{N}^{\prime}: \\
\left\|x \circ i_{N}^{M}\right\|_{M, m}^{*}\left\|y \circ i_{N}^{M}\right\|_{M, m}^{*} \leq \varepsilon\|x\|_{N, n}^{*}\|y\|_{N, n}^{*}+S\left\|x \circ i_{N}^{K}\right\|_{K, k}^{*}\left\|y \circ i_{N}^{K}\right\|_{K, k}^{*} .
\end{gathered}
$$

Remarks. (1) Each one of the conditions imply that

$$
\operatorname{Proj}^{1} X=\operatorname{Proj}^{1} E=0
$$

(comp. [83, 3.2.18]).

(2) Assumptions (a) and (b) are needed only to prove (3) $\Rightarrow(1)$ or $(2) \Rightarrow(1)$.

(3) Our result is much more general than the known results when one of the tensorized spaces is metrizable and the other dual metrizable (Vogt [78], Frerick [28], Frerick-Wengenroth [30, 74]) or recent results of Bonet and the author $[6,8]$.

(4) By $[8$, Th. 3.1,3.4], the result is also true if one of the spaces $X$ or $E$ is a nuclear Fréchet space and, by [6, Th. 4.1], if one of the spaces is an LNspace. In [6] and [8] one of the spaces is represented by a "linearly ordered" family of Banach spaces - lack of such a "linear order" is the main difficulty in the proof below. We need the result as above in Theorem 5.2 having in mind applications to highly non-metrizable spaces $\mathscr{A}(U)$.

(5) Another approach to vanishing of $\operatorname{Proj}^{1}$ on complicated spaces is given in [29].

(6) We use a convention $0 \cdot \infty=0$.

Proof of Theorem 5.2. (1) $\Rightarrow(2)$ : Apply [83, Th. 3.2.18 or Th. 3.3.6] for functionals of the form $x \otimes y \in\left(X_{N} \varepsilon E_{N}\right)^{\prime}$.

$(2) \Rightarrow(3)$ : The proof is inspired by $[8$, Th. $3.1(2) \Rightarrow(3)]$. Condition $(T)$ implies by Braun and Vogt's lemma in the form given in [6, Lemma 4.4], the condition $\left(P_{X}\right)$ and $\left(P_{Y}\right)$ respectively:

$$
\begin{aligned}
\forall N_{1} \exists N \geq & N_{1} \forall K \geq N \exists n_{1} \forall n \geq n_{1}, \gamma_{X}>0 \exists C_{X}, k \geq n \\
& \forall x \in X_{N_{1}}^{\prime}\left\|x \circ i_{N_{1}}^{N}\right\|_{N, n}^{*} \leq C_{X}\left\|x \circ i_{N_{1}}^{K}\right\|_{K, k}^{*}+\gamma_{X}\|x\|_{N_{1}, n_{1}}^{*}, \\
\forall \tilde{N}_{1} \exists \tilde{N} \geq & \tilde{N}_{1} \forall \tilde{K} \geq \tilde{N} \exists \tilde{n}_{1} \forall \tilde{n} \geq \tilde{n}_{1}, \tilde{\gamma}_{Y}>0 \exists C_{Y}, \tilde{k} \geq \tilde{n} \\
& \forall y \in E_{\tilde{N}_{1}}^{\prime}\left\|y \circ i_{\tilde{N}_{1}}^{\tilde{N}}\right\|_{\tilde{N}, \tilde{n}}^{*} \leq C_{Y}\left\|y \circ i_{\tilde{N}_{1}}^{\tilde{K}_{1}}\right\|_{\tilde{K}, \tilde{k}}^{*}+\gamma_{Y}\|y\|_{\tilde{N}_{1}, \tilde{n}_{1}}^{*} .
\end{aligned}
$$

Assume $(T)$ in the form:

$$
\begin{gathered}
\forall N, \tilde{N} \exists M \geq N, \tilde{M} \geq \tilde{N} \forall K \geq M, \tilde{K} \geq \tilde{M} \exists n, \tilde{n} \forall m, \tilde{m} \exists S, k, \tilde{k} \\
\forall x \in X_{N}^{\prime}, y \in E_{\tilde{N}}^{\prime}: \\
\left\|x \circ i_{N}^{M}\right\|_{M, m}^{*}\left\|y \circ i_{\tilde{N}}^{\tilde{M}}\right\|_{\tilde{M}, \tilde{m}}^{*} \leq S\left(\|x\|_{N, n}^{*}\|y\|_{\tilde{N}, \tilde{n}}^{*}+\left\|x \circ i_{N}^{K}\right\|_{K, k}^{*}\left\|y \circ i_{\tilde{N}}^{\tilde{K}}\right\|_{\tilde{K}, \tilde{k}}^{*}\right)
\end{gathered}
$$


We take $x \in X_{N_{1}}^{\prime}, y \in E_{\tilde{N}_{1}}^{\prime}$. We apply $(T)$ to $x \circ i_{N_{1}}^{N}, y \circ i_{\tilde{N}_{1}}^{\tilde{N}}$ and obtain the condition $\left(T_{1}\right)$ :

$$
\begin{aligned}
& \left\|x \circ i_{N_{1}}^{N} \circ i_{N}^{M}\right\|_{M, m}^{*}\left\|y \circ i_{\tilde{N}_{1}}^{\tilde{N}} \circ i_{\tilde{N}}^{\tilde{N}}\right\|_{\tilde{M}, \tilde{m}}^{*} \leq \\
& \quad \leq S\left(\left\|x \circ i_{N_{1}}^{N}\right\|_{N, n}^{*}\left\|y \circ i_{\tilde{N}_{1}}^{\tilde{N}}\right\|_{\tilde{N}, \tilde{n}}^{*}+\left\|x \circ i_{N_{1}}^{N} \circ i_{N}^{K}\right\|_{K, k}^{*}\left\|y \circ i_{\tilde{N}_{1}}^{\tilde{N}} \circ i_{\tilde{N}}^{\tilde{K}}\right\|_{\tilde{K}, \tilde{k}}^{*}\right) .
\end{aligned}
$$

If $\left\|y \circ i_{\tilde{N}_{1}}^{\tilde{N}}\right\|_{\tilde{N}, \tilde{n}}^{*} \leq\left\|y \circ i_{\tilde{N}_{1}}^{\tilde{K}}\right\|_{\tilde{K}, \tilde{k}}^{*}$ then we apply $\left(P_{X}\right)$ to $\left(T_{1}\right)$ and choose $\gamma_{X} \leq$ $\varepsilon /(2 S)$. Otherwise we apply first $\left(P_{Y}\right)$ to $\left(T_{1}\right)$ and then $\left(P_{X}\right)$ to the term with constant $S C_{Y}$ and take $\gamma_{Y} \leq \varepsilon /(2 S), \gamma_{X} \leq \varepsilon /\left(2 S C_{Y}\right)$. This completes the proof of $(2) \Rightarrow(3)$ (details are left to the reader).

Before we prove the implication $(3) \Rightarrow(1)$ we need some auxiliary results.

Proposition 5.3 If a pair of $P L S$-spaces $(X, E)$ satisfies $\left(T_{\varepsilon}\right)$ then if $\left(E_{N, n}\right)$ are chosen to be reflexive for all $N, n$ the same holds for all $y \in E_{N, k}^{\prime}$ and the pair $(X, E)$ satisfies also $\left(T B_{\varepsilon}\right)$, i.e.,

$$
\begin{gathered}
\forall N, \tilde{N} \exists M \geq N, \tilde{M} \geq \tilde{N} \forall K \geq M, \tilde{K} \geq \tilde{M} \quad \exists n, \tilde{n} \forall m \geq n, \tilde{m} \geq \tilde{n}, \\
\forall \varepsilon>0 \exists k \geq m, \tilde{k} \geq \tilde{m}, S \forall y \in E_{\tilde{N}, \tilde{k}}^{\prime}: \\
\left\|y \circ i_{\tilde{N}}^{\tilde{M}}\right\|_{\tilde{M}, \tilde{m}}^{*} i_{N}^{M} B_{M, m} \subseteq \varepsilon\|y\|_{\tilde{N}, \tilde{n}}^{*} B_{N, n}+S\left\|y \circ i_{\tilde{N}}^{\tilde{K}}\right\|_{\tilde{K}, \tilde{k}}^{*} i_{N}^{K} B_{K, k} .
\end{gathered}
$$

Moreover, if $E$ is deeply reduced the above condition holds for $y \in \overline{E_{\tilde{N}, \tilde{k}}^{\prime}}$, where the closure is taken with respect to the standard topology of $E_{\tilde{N}, \tilde{n}}^{\prime} \cap E_{\tilde{K}, \tilde{k}}^{\prime}$.

Proof. By $[8$, Lemma $3.5(\mathrm{~b})]$, we obtain from $\left(T_{\varepsilon}\right)$ the condition $\left(T B_{\varepsilon}\right)$ but only for $y \in E_{\tilde{N}}^{\prime}$.

Since $E_{\tilde{N}, \tilde{k}} \hookrightarrow E_{\tilde{N}}$ is injective and $E_{\tilde{N}, \tilde{k}}, E_{\tilde{N}}$ can be assumed reflexive, the dual map $\pi_{\tilde{k}}: E_{\tilde{N}}^{\prime} \rightarrow E_{\tilde{N}, \tilde{k}}^{\prime}$ has a dense image. We define analogously $\pi_{\tilde{n}}^{\tilde{k}}: E_{\tilde{N}, \tilde{k}}^{\prime} \rightarrow E_{\tilde{N}, \tilde{n}}^{\prime}$. Let us take $z \in E_{\tilde{N}, \tilde{k}}^{\prime}, v \in B_{M, m}$. Then there is a sequence $\left(y_{j}\right) \subseteq E_{\tilde{N}}^{\prime}$ such that $\pi_{\tilde{k}} y_{j} \rightarrow z$ in $E_{\tilde{N}, \tilde{k}}^{\prime}$. We get

$$
\left\|\pi_{\tilde{m}}^{\tilde{k}} \pi_{\tilde{k}} y_{j} \circ i_{\tilde{N}}^{\tilde{M}}\right\|_{\tilde{M}, \tilde{m}}^{*} i_{N}^{M} v=\varepsilon\left\|\pi_{\tilde{n}}^{\tilde{k}} \pi_{\tilde{k}} y_{j}\right\|_{\tilde{N}, \tilde{n}}^{*} a_{j}+S\left\|\pi_{\tilde{k}} y_{j} \circ i_{\tilde{N}}^{\tilde{K}}\right\|_{\tilde{K}, \tilde{k}}^{*} i_{N}^{K} b_{j},
$$

where $\left(a_{j}\right) \subseteq B_{N, n},\left(b_{j}\right) \subseteq B_{K, k}$. Since sets $B_{N, n}, B_{K, k}$ are compact in $X_{N}$ and $X_{K}$, and since every compact set of an LS-space is metrizable [15], we can find a suitable subsequence of $j$ such that the corresponding subsequences of $\left(a_{j}\right)$ and $\left(b_{j}\right)$ are convergent to $a \in B_{N, n}$ and $b \in B_{K, k}$. We have proved that

$$
\begin{aligned}
& \forall N, \tilde{N} \exists M \geq N, \tilde{M} \geq \tilde{N} \forall K \geq M, \tilde{K} \geq \tilde{M} \\
& \exists n, \tilde{n} \forall m \geq n, \tilde{m} \geq \tilde{n}, \varepsilon>0 \quad \exists k \geq m, \tilde{k} \geq \tilde{m}, \quad \exists S>0 \forall z \in E_{\tilde{N}, \tilde{k}}^{\prime} \\
& \quad\left\|\pi_{\tilde{m}}^{\tilde{k}} z \circ i_{\tilde{N}}^{\tilde{M}}\right\|_{\tilde{M}, \tilde{m}}^{*} i_{N}^{M} B_{M, m} \subseteq \varepsilon\left\|\pi_{\tilde{n}}^{\tilde{k}} z\right\|_{\tilde{N}, \tilde{n}}^{*} B_{N, n}+S\left\|z \circ i_{\tilde{N}}^{\tilde{K}}\right\|_{\tilde{K}, \tilde{k}}^{*} i_{N}^{K} B_{K, k} .
\end{aligned}
$$


If $E$ is deeply reduced then we may (and we will) assume that $E_{\tilde{N}, \tilde{k}}^{\prime}$ injectively embeds continuously into $E_{\tilde{N}, \tilde{n}}^{\prime}$ and $E_{\tilde{K}, \tilde{k}}^{\prime}$. Clearly, then for $z \in \overline{E_{\tilde{N}, \tilde{k}}^{\prime}}$ we can find $y_{j} \in E_{\tilde{N}, \tilde{k}}^{\prime}$ which tends to $z$ both in the norm of $E_{\tilde{N}, \tilde{n}}^{\prime}$ and $E_{\tilde{K}, \tilde{k}}^{\prime}$. The same argument as above shows the required inequality.

The first statement can be proved by the same trick.

Proposition 5.4 If a pair $(X, E)$ of PLS-spaces satisfies $\left(T_{\varepsilon}\right)$ for strongly reduced projective representations of $X, E$ then either $X$ is strict or $E$ is deeply reduced in rows and either $E$ is deeply reduced in columns or $X$ is a Fréchet space.

Proof. Let us assume that $E$ is not deeply reduced in rows. Hence

$$
\exists N \forall M \forall n \exists m \forall k \exists y \in E_{N, k}^{\prime} \quad \pi_{m}^{k}\left(y \circ i_{N}^{M}\right) \neq 0 \quad \text { but } \quad \pi_{n}^{k} y=0,
$$

where $\pi_{m}^{k}: E_{M, k}^{\prime} \rightarrow E_{M, m}^{\prime}, \pi_{n}^{k}: E_{N, k}^{\prime} \rightarrow E_{N, n}^{\prime}$ are restriction maps. By Proposition 5.3 , the condition $\left(T B_{\varepsilon}\right)$ holds, so we get

$$
\forall N \exists M \geq N \forall K \geq M \forall m \exists k \geq m, S \quad i_{N}^{M} B_{M, m} \subseteq S i_{N}^{K} B_{K, k} .
$$

Therefore

$$
\forall N \exists M(N) \geq N \forall K \geq M \quad i_{N}^{M} X_{M} \subseteq i_{N}^{K} X_{K}
$$

We get inductively a sequence $\left(N_{j}\right), N_{j+1}=M\left(N_{j}\right)$, and the strict spectrum $\left(X_{N_{j}} /\right.$ ker $\left.i_{N_{j-1}}^{N_{j}}\right)$ is equivalent to the given one.

If $X$ is not a Fréchet space, then

$$
\begin{gathered}
\exists N_{0} \forall N \geq N_{0} \forall M \geq N \forall n \exists m_{0} \forall m \geq m_{0} \exists\left(x_{j}\right) \subseteq X_{N}^{\prime} \\
\frac{\left\|x_{j} \circ i_{N}^{M}\right\|_{M, m}^{*}}{\left\|x_{j}\right\|_{N, n}^{*}} \rightarrow \infty \quad \text { for } j \rightarrow \infty .
\end{gathered}
$$

Let us take $\left(T_{\varepsilon}\right)$ and apply it for $x_{j}$ and $y \in E_{N, k}^{\prime}$ such that $\left.y \circ i_{N}^{K}\right|_{E_{K, k}}=0$ by Prop. 5.3. Thus for every $j>0$ we get

$$
\left\|y \circ i_{N}^{M}\right\|_{M, m}^{*} \cdot\left\|x_{j} \circ i_{N}^{M}\right\|_{M, m}^{*} \leq \varepsilon\|y\|_{N, n}^{*} \cdot\left\|x_{j}\right\|_{N, n}^{*} .
$$

We have proved that for any $\varepsilon>0$

$$
\left\|y \circ i_{N}^{M}\right\|_{M, m}^{*} \leq \varepsilon\|y\|_{N, n}^{*} .
$$

If $y \in E_{N, k}^{\prime}$ belongs to the polar of $\varepsilon B_{N, n}+i_{N}^{K} E_{K, k}$ then it belongs to the polar of $i_{N}^{M} B_{M, m}$. Therefore, for every $\varepsilon>0$ we get

$$
i_{N}^{M} B_{M, m} \subseteq{\overline{\varepsilon B_{N, n}+i_{N}^{K} E_{K, k}}}_{E_{N, k}} .
$$


Since $B_{N, n} \subseteq B_{N, k}$ we get

$$
i_{N}^{M} B_{M, m} \subseteq{\overline{i_{N}^{K} E_{K, k}}}^{E_{N, k}} .
$$

We have proved that $E$ is deeply reduced in columns.

Next result is a slight modification of [21, Lemma 2.2] (originally the result is also in [61]). We omit the proof based on the spectral theory of unbounded operators since it is a modification of the proof in [21, Lemma 2.2].

Theorem 5.5 Let $H, H_{0}, H_{1}$ be Hilbert spaces and let $U$ be a topological vector space. Let us assume that the following diagram of injective continuous linear maps commutes

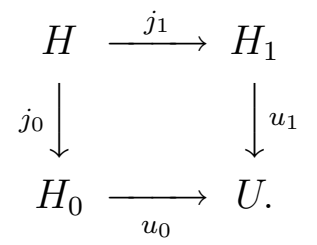

Let $j_{1}(H)$ and $j_{0}(H)$ be dense in $H_{1}$ and $H_{0}$ respectively. Moreover, let $H$ be complete in the norm:

$$
\|x\|:=\left(\left\|j_{1} x\right\|_{H_{1}}^{2}+\left\|j_{0} x\right\|_{H_{0}}^{2}\right)^{1 / 2} .
$$

Then for any $\varepsilon>0$ there are: a set $I$, positive weights $v: I \rightarrow \mathbb{R}_{+}, w: I \rightarrow \mathbb{R}_{+}$ and isomorphisms

$$
T: H \rightarrow \ell_{2}(I), \quad V: H_{0} \rightarrow \ell_{2}(I, v), \quad W: H_{1} \rightarrow \ell_{2}(I, w)
$$

such that the following diagram commutes:

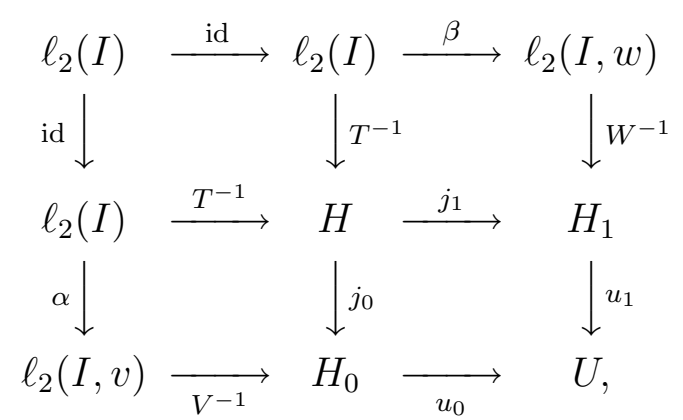

where $\alpha, \beta$ are natural embeddings and

$$
\|T\| \cdot\left\|T^{-1}\right\|,\|V\| \cdot\left\|V^{-1}\right\|,\|W\| \cdot\left\|W^{-1}\right\|<1+\varepsilon .
$$


Proof of Theorem 5.2. $(3) \Rightarrow(1)$ : (a): Assume that $E$ is a deeply reduced PLN-space. Without loss of generality we may assume that for every $N$, $n \in \mathbb{N}$ big enough:

- $E_{N, n}^{\prime}$ is a Hilbert space;

- $E_{N, n}^{\prime} \rightarrow E_{N+1, n}^{\prime}$ and $E_{N, n+1}^{\prime} \rightarrow E_{N, n}^{\prime}$ are injective Hilbert-Schmidt maps of the Hilbert-Schmidt norm $\nu_{2}$ and operator norm $\leq 1$;

- $E_{N, n+1}^{\prime}$ is dense in $E_{N, n}^{\prime}$ (since $E_{N, n} \hookrightarrow E_{N, n+1}$ is injective).

Applying $\left(T_{\varepsilon}\right)$ we get, by Proposition 5.3, for every $y \in \overline{E_{N, k}^{\prime}} E_{N, n}^{\prime} \cap E_{K, k}^{\prime}$ the following inclusion:

$$
\left\|y \circ i_{N}^{M}\right\|_{M, m}^{*} i_{N}^{M} B_{M, m} \subseteq \varepsilon\|y\|_{N, n}^{*} B_{N, n}+S\left\|y \circ i_{N}^{K}\right\|_{K, k}^{*} i_{N}^{K} B_{K, k} .
$$

We define

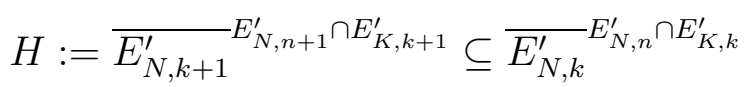

here we equip $H$ with the Hilbert norm from the intersection space $E_{N, n+1}^{\prime} \cap$ $E_{K, k+1}^{\prime}$. Since

$$
E_{N, k+1}^{\prime} \subseteq H_{1}:=\left(\operatorname{ker}\left(\left.i_{N}^{K}\right|_{E_{K, k+1}}\right)\right)^{\perp} \subseteq E_{K, k+1}^{\prime}
$$

we have

$$
H={\overline{E_{N, k+1}^{\prime}}}_{E_{N, n+1}^{\prime} \cap H_{1}}
$$

and the image of $H$ is dense in $H_{1}$. Obviously $H$ is dense in $H_{0}:=E_{N, n+1}^{\prime}$. If we define $U=E_{K, n}^{\prime}$ then we are exactly in the situation described in Theorem 5.5.

The natural maps

$$
\pi_{0}: H_{0} \rightarrow E_{N, n}^{\prime}, \quad \pi_{1}: H_{1} \rightarrow E_{K, k}^{\prime}
$$

are Hilbert-Schmidt injections of Hilbert-Schmidt norm $\leq 1$. Moreover, by Prop. 5.3, the natural map $E_{N, k+1}^{\prime} \rightarrow E_{M, m}^{\prime}$ is continuous when the domain space is equipped with the Hilbert norm of $E_{N, n+1}^{\prime} \cap E_{K, k+1}^{\prime}$. It extends to a continuous linear map $r: H \rightarrow E_{M, m}^{\prime}$.

Let $R \in W\left(U_{M, m}, B_{M, m}\right)$. Using the map $T$ obtained via Theorem 5.5, we define

$$
t_{i}:=R\left(r T^{-1}\left(e_{i}\right)\right) \in\left\|r T^{-1} e_{i}\right\|_{M, m}^{*} B_{M, m} .
$$

Using (5.2) we find

$$
\tilde{w}_{i} \in S\left\|\pi_{1} j_{1} T^{-1}\left(e_{i}\right)\right\|_{K, k}^{*} B_{K, k}, \quad \tilde{v}_{i} \in \varepsilon\left\|\pi_{0} j_{0} T^{-1}\left(e_{i}\right)\right\|_{N, n}^{*} B_{N, n}
$$

such that

$$
i_{N}^{M} t_{i}=i_{N}^{K} \tilde{w}_{i}+\tilde{v}_{i}, \quad \text { for all } i
$$


We define

$$
\tilde{W}: H_{1} \rightarrow X_{K, k}, \quad \tilde{W}(x):=\sum_{i} \frac{1}{w_{i}^{2}}\left\langle W x, e_{i}\right\rangle_{\ell_{2}(I, w)} \tilde{w}_{i}
$$

and

$$
\tilde{V}: H_{0} \rightarrow X_{N, n}, \quad \tilde{V}(x):=\sum_{i} \frac{1}{v_{i}^{2}}\left\langle V x, e_{i}\right\rangle_{\ell_{2}(I, v)} \tilde{v}_{i} .
$$

We will show that they are well defined and continuous. Let $x \in H_{1}$,

$$
\begin{aligned}
& \|\tilde{W}(x)\|_{K, k} \leq \sum_{i}\left|\frac{1}{w_{i}^{2}}\left\langle W x, e_{i}\right\rangle_{\ell_{2}(I, w)}\right| \cdot\left\|\tilde{w}_{i}\right\|_{K, k} \\
& \leq S \sum_{i}\left|\left\langle W x, \frac{e_{i}}{w_{i}}\right\rangle_{\ell_{2}(I, w)}\right| \cdot\left\|\pi_{1} j_{1} T^{-1}\left(\frac{e_{i}}{w_{i}}\right)\right\|_{K, k} \\
& \leq S\left(\sum_{i}\left|\left\langle W x, \frac{e_{i}}{w_{i}}\right\rangle_{\ell_{2}(I, w)}\right|^{2}\right)^{1 / 2} \cdot\left(\sum_{i}\left\|\pi_{1} j_{1} T^{-1}\left(\frac{e_{i}}{w_{i}}\right)\right\|_{K, k}^{2}\right)^{1 / 2} \\
& =S\|W x\|_{\ell_{2}(I, w)} \nu_{2}\left(\pi_{1} \circ W^{-1}\right) \leq S(1+\varepsilon)\|x\|_{H_{1}}(1+\varepsilon) \nu_{2}\left(\pi_{1}\right) \text {. }
\end{aligned}
$$

Analogously, for $x \in H_{0}$,

$$
\begin{aligned}
\|\tilde{V}(x)\|_{N, n} & \leq \sum_{i}\left|\frac{1}{v_{i}^{2}}\left\langle V x, e_{i}\right\rangle_{\ell_{2}(I, v)}\right| \cdot\left\|\pi_{0} j_{0} T^{-1}\left(\frac{e_{i}}{v_{i}}\right)\right\|_{N, n}^{*} \\
& \leq \varepsilon(1+\varepsilon)\|x\|_{H_{0}}(1+\varepsilon) \nu_{2}\left(\pi_{0}\right) .
\end{aligned}
$$

We have proved that

$$
\|\tilde{W}\| \leq S(1+\varepsilon)^{2}, \quad\|\tilde{V}\| \leq \varepsilon(1+\varepsilon)^{2} .
$$

Since $H_{1}$ is a closed Hilbert subspace of $E_{K, k+1}^{\prime}$ we can extend $\tilde{W}$ with the same norm onto $E_{K, k+1}^{\prime}$.

Let us take $x \in E_{N}^{\prime} \subseteq E_{N, k+1}^{\prime} \subseteq H$. We calculate

$$
\begin{aligned}
i_{N}^{M} \circ R \circ\left(i_{N}^{M}\right)^{\prime} x & =i_{N}^{M} \circ R \circ r x=i_{N}^{M} \circ R \circ r \circ T^{-1} \circ T x \\
& =\sum_{i} i_{N}^{M} \circ R \circ r \circ T^{-1}\left(e_{i}\right)\left\langle T x, e_{i}\right\rangle_{\ell_{2}(I)}=\sum_{i} i_{N}^{M} t_{i}\left\langle T x, e_{i}\right\rangle_{\ell_{2}(I)} \\
& =\sum_{i} i_{N}^{K} \tilde{w}_{i}\left\langle T x, e_{i}\right\rangle_{\ell_{2}(I)}+\sum_{i} \tilde{v}_{i}\left\langle T x, e_{i}\right\rangle_{\ell_{2}(I)} \\
& =\sum_{i} i_{N}^{K} \tilde{w}_{i}\left\langle W j_{1} x, e_{i}\right\rangle_{\ell_{2}(I)}+\sum_{i} \tilde{v}_{i}\left\langle V j_{0} x, e_{i}\right\rangle_{\ell_{2}(I)} \\
& =i_{N}^{K} \tilde{W}\left(j_{1} x\right)+\tilde{V}\left(j_{0} x\right) .
\end{aligned}
$$


We have proved that

$$
\begin{gathered}
i_{N}^{M} \circ W\left(U_{M, m}, B_{M, m}\right) \circ\left(i_{N}^{M}\right)^{\prime} \subseteq S(1+\varepsilon)^{2} i_{N}^{K} \circ W\left(U_{K, k+1}, B_{K, k}\right) \circ\left(i_{N}^{K}\right)^{\prime} \\
+\varepsilon(1+\varepsilon)^{2} W\left(U_{N, n+1}, B_{N, n}\right) .
\end{gathered}
$$

By Theorem 4.2 and [83, Th. 3.2.14], this implies $\operatorname{Proj}^{1}(X \varepsilon E)=0$.

(b): By the Remark (4) after Theorem 5.2, we may assume that none of the spaces is Fréchet. If both are strict then as in the proof of Proposition 4.5 we show surjectivity of

$$
i_{N}^{N+1} \otimes i_{N}^{N+1}: X_{N+1} \varepsilon E_{N+1} \rightarrow X_{N} \varepsilon E_{N} .
$$

Thus the spectrum is strict and automatically $\operatorname{Proj}^{1} X \varepsilon E=0$. We may assume that $X$ is neither strict nor Fréchet, then Prop. 5.4 implies that $E$ is deeply reduced. Apply part (a).

(c): By Theorem 4.2 and [83, Th. 3.2.14], we need to prove

$$
\begin{array}{r}
\forall N, \tilde{N}, \quad \exists M \geq N, \tilde{M} \geq \tilde{N} \forall K \geq M, \tilde{K} \geq \tilde{M} \\
\exists n, \tilde{n} \forall m \geq n, \tilde{m} \geq \tilde{n}, \varepsilon>0 \quad \exists k \geq m, \tilde{k} \geq \tilde{m}, \quad \exists S>0 \\
i_{N}^{M} \circ W\left(U_{\tilde{M}, \tilde{m}}, B_{M, m}\right) \circ\left(i_{\tilde{N}}^{M}\right)^{\prime} \subseteq S i_{N}^{K} \circ W\left(U_{\tilde{K}, \tilde{k}}, B_{K, k}\right) \circ\left(i_{\tilde{N}}\right)^{\prime}+ \\
+\varepsilon W\left(U_{\tilde{N}, \tilde{n}}, B_{N, n}\right) .
\end{array}
$$

We assume first that $E=\Lambda^{\infty}(B), b_{1, n}(i)>0$ for each $n$. Let $e_{i}$ be the unit vector in $E^{\prime}$, then $\left\|e_{i}\right\|_{N, n}^{*}=1 / b_{N, n}(i)$. Thus, by $\left(T_{\varepsilon}\right)$ for $y=e_{i}$ and $x \in X_{N}^{\prime}$ we get

$$
\frac{\left\|x \circ i_{N}^{M}\right\|_{M, m}^{*}}{b_{\tilde{M}, \tilde{m}}(i)} \leq \varepsilon \frac{\|x\|_{N, n}^{*}}{b_{\tilde{N}, \tilde{n}}(i)}+S \frac{\left\|x \circ i_{N}^{K}\right\|_{K, k}^{*}}{b_{\tilde{K}, \tilde{k}}(i)} .
$$

By [8, Lemma 3.5], we get

$$
\frac{i_{N}^{M} B_{M, m}}{b_{\tilde{M}, \tilde{m}}(i)} \subseteq \frac{2 \varepsilon B_{N, n}}{b_{\tilde{N}, \tilde{n}}(i)}+\frac{2 S i_{N}^{K} B_{K, k}}{b_{\tilde{K}, \tilde{k}}(i)} .
$$

We identify

$$
W\left(U_{\tilde{M}, \tilde{m}}, B_{M, m}\right) \subseteq L\left(E_{\tilde{M}}^{\prime}, X_{M}\right)
$$

with a subset of the space of vector valued sequences:

$L\left(E_{\tilde{M}}^{\prime}, X_{M}\right)=\left\{u=(u(i))_{i \in \mathbb{N}} \subseteq X_{M}: \exists m, \tilde{m} \sup _{i} b_{\tilde{M}, \tilde{m}}(i)\|u(i)\|_{M, m}<\infty\right\}$.

In particular,

$$
u=(u(i))_{i \in \mathbb{N}} \in W\left(U_{\tilde{M}, \tilde{m}}, B_{M, m}\right)
$$

if and only if

$$
u(i) \in\left(b_{\tilde{M}, \tilde{m}}(i)\right)^{-1} B_{M, m} \text { for every } i \in \mathbb{N} .
$$


By (5.4), we get

$$
i_{N}^{M} u(i)=2 \varepsilon v(i)+2 S i_{N}^{K} w(i) \quad \text { for each } i \in \mathbb{N},
$$

where

$$
v(i) \in\left(b_{\tilde{N}, \tilde{n}}(i)\right)^{-1} B_{N, n}, \quad w(i) \in\left(b_{\tilde{K}, \tilde{k}}(i)\right)^{-1} B_{K, k} .
$$

We define

$$
v \in W\left(U_{\tilde{N}, \tilde{n}}, B_{N, n}\right) \subseteq L\left(E_{\tilde{N}}^{\prime}, X_{N}\right), \quad w \in W\left(U_{\tilde{K}, \tilde{k}}, B_{K, k}\right) \subseteq L\left(E_{\tilde{K}}^{\prime}, X_{K}\right)
$$

by

$$
v(x):=(v(i) x(i))_{i \in \mathbb{N}}, \quad w(z):=(w(i) z(i))_{i \in \mathbb{N}},
$$

for

$$
x=(x(i)) \in E_{\tilde{N}}^{\prime}, \quad z=(z(i)) \in E_{\tilde{K}}^{\prime} .
$$

Obviously,

$$
i_{N}^{M} \circ u \circ\left(i_{\tilde{N}}^{\tilde{M}}\right)^{\prime}=2 \varepsilon v+2 S i_{N}^{K} \circ w \circ\left(i_{\tilde{N}}^{\tilde{K}}\right)^{\prime},
$$

which implies (5.3) with changed $\varepsilon$ and $S$.

In the general case $E=\Lambda^{\infty}(B)$ is a countable product of spaces $E^{(j)}$ for which we have proved $\operatorname{Proj}^{1}\left(X \varepsilon E^{(j)}\right)=0$. Apply Proposition 4.8.

Next corollary is a generalization of the famous $(D N)-(\Omega)$ splitting theorem [57, Th. 30.1] or [82, Satz 1.9] which means, in particular, that if $E$ is an LS-space, $E^{\prime} \in(D N)$, and $X$ is a Fréchet Schwartz space, $X \in(\Omega)$, then $\operatorname{Proj}^{1}\left(X \varepsilon E^{\prime}\right)=0$ whenever one of the spaces is nuclear. Observe that in that case both $E$ and $X$ have the dual interpolation estimate for big $\theta$. It also generalizes results of Grothendieck [32, II.§4, no. 3, Prop.15] and Vogt [77, Th. 4.9].

Corollary 5.6 Let $X, E$ be PLS-spaces. Assume that both spaces are PLNspaces or one of them is a deeply reduced PLN-space. If $E$ and $X$ have the dual interpolation estimate for small (big) $\theta$ then $L_{b}\left(E_{b}^{\prime}, X\right)=X \varepsilon E$ is ultrabornological and $\operatorname{Proj}^{1}(X \varepsilon E)=0$.

Remark. Results on inheritance of dual interpolation estimates for Schwartz (tensor) products of PLS-spaces were very recently obtained by Piszczek using the results of this paper, see [67].

Proof. Assume that

$$
\begin{array}{r}
\left.\forall N \exists M \geq N \forall K \geq M \exists n \forall m \geq n \exists \theta_{0} \forall \theta \in\right] 0, \theta_{0}[\exists k, C \\
\forall x \in X_{N}^{\prime}, y \in E_{N}^{\prime} \\
\left\|x \circ i_{N}^{M}\right\|_{M, m}^{*} \leq C\left(\|x\|_{N, n}^{*}\right)^{\theta} \cdot\left(\left\|x \circ i_{N}^{K}\right\|_{K, k}^{*}\right)^{1-\theta} \\
\left\|y \circ i_{N}^{M}\right\|_{M, m}^{*} \leq C\left(\|y\|_{N, n}^{*}\right)^{\theta} \cdot\left(\left\|y \circ i_{N}^{K}\right\|_{K, k}^{*}\right)^{1-\theta} .
\end{array}
$$

Multiplying this inequalities we get easily $(T)$. Apply Theorems 5.2 and 4.1. 


\section{Conditions with one fixed space}

The previous section gave a characterization of vanishing of $\operatorname{Proj}^{1} X \varepsilon E$ for a large class of PLS-spaces $E, X$. We fix one space $E$ and we characterize those spaces $X$ such that $\operatorname{Proj}^{1} X \varepsilon E=0$

Corollary 6.1 Let $X$ be a PLS-space, $U$ a real analytic manifold, then $\operatorname{Proj}^{1} X \varepsilon \mathscr{A}(U)=0$ if and only if $X$ satisfies the dual interpolation estimate for small $\theta$ (for $U$ connected non-compact) or if and only if $X$ satisfies $(P \overline{\bar{\Omega}})$ (for $U$ compact).

Proof. Sufficiency for the non-compact case follows from Corollary 5.6 and 2.3 since $\mathscr{A}(U)$ is deeply reduced. For the compact case use Proposition 2.1 and [6, Cor. 3.8, 7.2].

Necessity. The function theoretic argument from the proof of [6, Th. 7.8] gives numbers $1>a>b$ and a sequence of functionals $\left(g_{j}\right) \subset \mathscr{A}(\mathbb{R})^{\prime}$ such that for $j \in \mathbb{N}$

$$
1=\left\|g_{j}\right\|_{N, n}^{*}>a^{j}=\left\|g_{j}\right\|_{M, m}^{*}>b^{j}=\left\|g_{j}\right\|_{K, k}^{*} .
$$

Since, by Proposition 2.1, $\mathscr{A}(\mathbb{R})$ is a quotient of $\mathscr{A}(U)$ for non-compact connected $U$ then we may assume that $\left(g_{j}\right) \subset \mathscr{A}(U)^{\prime}$. Plugging the constructed functions into the condition ( $\mathrm{T}$ ) from Theorem 5.2 we get analogously as in the proof of $\left[6\right.$, Th. 7.8] that for some $\eta>0$, big $r$ and all $y \in X_{N}^{\prime}$

$$
\left\|y \circ i_{N}^{M}\right\|_{M, m}^{*} \leq C\left(\left\|y \circ i_{N}^{K}\right\|_{K, k}^{*} r^{-\eta}+\|y\|_{N, n}^{*} r\right)
$$

which is equivalent to $(P \underline{A})$.

On the other hand, $\mathscr{A}(U) \stackrel{\text { comp }}{\supseteq} \mathscr{A}(\mathbb{T}) \simeq \Lambda_{0}^{\prime}(j)$ (see the proof of Proposition 2.1 and [19, Th. 5.4]) thus $\operatorname{Proj}^{1} X \varepsilon \Lambda_{0}^{\prime}(j)=0$. From [6, Cor. 3.8, 7.2] it follows that $X$ has $(P \overline{\bar{\Omega}})$.

Corollary 6.2 Let $X$ be a PLS-space, $\Omega \subseteq \mathbb{R}^{d}$ be an arbitrary open set, $\omega$ non-quasianalytic. $\operatorname{Proj}^{1} X \varepsilon \mathscr{E}_{\{\omega\}}(\Omega)=0$ if and only if $X$ satisfies $(P \overline{\bar{\Omega}})$. Analogously, $\operatorname{Proj}^{1} X \varepsilon \mathscr{D}_{(\omega)}^{\prime}(\Omega)=0$ if and only if $X$ satisfies $(P \Omega)$.

Proof. Apply Proposition 4.8 and [6, Cor. 7.2,3.8]. By [11, Prop. 4.9, 5.6],

$$
\mathscr{E}_{\{\omega\}}(\Omega) \simeq \prod \Lambda_{0}(\alpha)^{\prime}, \quad \mathscr{D}_{(\omega)}^{\prime}(\Omega) \simeq \prod \Lambda_{\infty}(\beta)^{\prime} .
$$

Remark. The same holds for the space of currents $\mathscr{D}^{\prime}(\Omega)$ over arbitrary $C^{\infty}$-manifold $\Omega$ instead of $\mathscr{D}_{(\omega)}^{\prime}(\Omega)$. In that case sufficiency follows from the 
remark after Corollary 2.3. Necessity follows from the fact that $\mathscr{D}^{\prime}(\mathbb{T})$ is isomorphic to a complemented subspace of $\mathscr{D}^{\prime}(\Omega)$. Clearly $\mathscr{D}^{\prime}(\mathbb{T})$ are just periodic currents on $\mathbb{R}$ so it is isomorphic to $\Lambda_{0}^{\prime}(\log j)$.

Now, let us apply the results on the vanishing of $\operatorname{Proj}^{1}$ to the surjectivity problem:

Corollary 6.3 Let $T: \mathscr{D}^{\prime}\left(\Omega_{1}\right) \rightarrow \mathscr{D}^{\prime}\left(\Omega_{1}\right), S: \mathscr{D}^{\prime}\left(\Omega_{2}\right) \rightarrow \mathscr{D}^{\prime}\left(\Omega_{2}\right)$ be surjective operators, $\Omega_{1}, \Omega_{2} \subseteq \mathbb{R}^{d}$ arbitrary open or $C^{\infty}$-manifolds. Then

$$
T \otimes S: \mathscr{D}^{\prime}\left(\Omega_{1} \times \Omega_{2}\right) \rightarrow \mathscr{D}^{\prime}\left(\Omega_{1} \times \Omega_{2}\right)
$$

is surjective if and only if $\operatorname{ker} T$ and $\operatorname{ker} S$ have $(P \Omega)$.

An analogous result holds for

$$
T: \mathscr{D}_{\left(\omega_{1}\right)}^{\prime}\left(\Omega_{1}\right) \rightarrow \mathscr{D}_{\left(\omega_{1}\right)}^{\prime}\left(\Omega_{1}\right) \quad \text { and } \quad S: \mathscr{D}_{\left(\omega_{2}\right)}^{\prime}\left(\Omega_{2}\right) \rightarrow \mathscr{D}_{\left(\omega_{2}\right)}^{\prime}\left(\Omega_{2}\right) .
$$

Proof. Apply Cor. 4.6, Cor. 6.2 and the remark below it. Observe that

$$
\operatorname{Proj}^{1}\left(\mathscr{D}_{\left(\omega_{1}\right)}^{\prime}\left(\Omega_{1}\right) \varepsilon \mathscr{D}_{\left(\omega_{2}\right)}^{\prime}\left(\Omega_{2}\right)\right)=0 \quad \text { by Cor. } 2.3 \text { and Cor. 5.6. }
$$

Next corollary (Theorem C) is crucial for the whole paper giving a criterion of the real analytic parameter dependence of linear operators on spaces of distributions or currents.

Corollary 6.4 Let $T: \mathscr{D}_{(\omega)}^{\prime}(\Omega) \rightarrow \mathscr{D}_{(\omega)}^{\prime}(\Omega)$ be a surjective operator then

$$
T \otimes \operatorname{id} \mathscr{A}(U): \mathscr{D}_{(\omega)}^{\prime}(\Omega) \varepsilon \mathscr{A}(U) \rightarrow \mathscr{D}_{(\omega)}^{\prime}(\Omega) \varepsilon \mathscr{A}(U)
$$

is surjective if and only if ker $T$ has the dual interpolation estimate for small $\theta$ (for non-compact connected $U$ ) or if and only if $\operatorname{ker} T$ has $(P \overline{\bar{\Omega}})$ (for compact $U$ ). Therefore surjectivity does not depend on the choice of an open set $U \subseteq \mathbb{R}^{n}$.

Let us emphasize that the result is true, for instance, for variable coefficients differential operators. The dual interpolation estimate (2.1) is a kind of a very strong abstract "Hadamard three circle theorem" as explained in Section 1.

Proof. Apply Cor. 4.6 and Cor. 6.1. Observe that

$$
\operatorname{Proj}^{1} \mathscr{D}_{(\omega)}^{\prime}(\Omega) \varepsilon \mathscr{A}(U)=0 \quad \text { by Cor. } 5.6 \text { and Cor. 2.3. }
$$

Corollary 6.5 Let $T: \mathscr{D}_{(\omega)}^{\prime}(\Omega) \rightarrow \mathscr{D}_{(\omega)}^{\prime}(\Omega)$ be a surjective operator and let $\omega_{1}$ be a non-quasianalytic weight. Then

$$
T \otimes \operatorname{id}_{\mathscr{E}_{\left\{\omega_{1}\right\}}}(U): \mathscr{D}_{(\omega)}^{\prime}(\Omega) \varepsilon \mathscr{E}_{\left\{\omega_{1}\right\}}(U) \rightarrow \mathscr{D}_{(\omega)}^{\prime}(\Omega) \varepsilon_{\left\{\omega_{1}\right\}}(U)
$$

is surjective if and only if $\operatorname{ker} T$ has $(P \overline{\bar{\Omega}})$.

Proof. Apply Cor. 4.6, Cor. 6.2 and Cor. 2.3. 
Clearly there are plenty of other results of that type - we leave their formulation to the reader. Just for fun we give the following example of surjectivity results for products of differential operators.

Corollary 6.6 Let

$$
\begin{aligned}
P_{1}(x, D) & :=\sum_{|\alpha| \leq m} a_{\alpha}(x) \partial_{x}^{\alpha}: \mathscr{D}^{\prime}\left(\Omega_{1}\right) \rightarrow \mathscr{D}^{\prime}\left(\Omega_{1}\right), \\
P_{2}(y, D) & :=\sum_{|\beta| \leq k} b_{\beta}(y) \partial_{y}^{\beta}: \mathscr{D}^{\prime}\left(\Omega_{2}\right) \rightarrow \mathscr{D}^{\prime}\left(\Omega_{2}\right)
\end{aligned}
$$

be two linear surjective partial differential operators, $x \in \Omega_{1}, y \in \Omega_{2}$. The operator

$P_{1}(x, D) P_{2}(y, D)=\sum_{|\alpha| \leq m,|\beta| \leq k} a_{\alpha}(x) b_{\beta}(y) \partial_{x}^{\alpha} \partial_{y}^{\beta}: \mathscr{D}^{\prime}\left(\Omega_{1} \times \Omega_{2}\right) \rightarrow \mathscr{D}^{\prime}\left(\Omega_{1} \times \Omega_{2}\right)$ $(x, y) \in \Omega_{1} \times \Omega_{2}$, is surjective if and only if ker $P_{1}(x, D)$ and ker $P_{2}(x, D)$ have $(P \Omega)$.

The above results are worth studying mostly if we have a method of evaluating the dual interpolation estimates or P-conditions for the kernel of the map. Using known descriptions of kernels of convolution operators we can prove the following characterizations:

Corollary 6.7 Let $\omega$ be a non-quasianalytic weight and let $\mu \in \mathscr{D}_{(\omega)}^{\prime}(\mathbb{R})$ be an ultradistribution with compact support. The convolution operator

$$
T_{\mu}: \mathscr{D}_{(\omega)}^{\prime}(\mathbb{R}, \mathscr{A}(U)) \rightarrow \mathscr{D}_{(\omega)}^{\prime}(\mathbb{R}, \mathscr{A}(U))
$$

is surjective if and only if

$$
T_{\mu}: \mathscr{D}_{(\omega)}^{\prime}(\mathbb{R}) \rightarrow \mathscr{D}_{(\omega)}^{\prime}(\mathbb{R})
$$

has a linear continuous right inverse.

Remark. A characterization of existence of right inverse for $T_{\mu}$ in terms of $\hat{\mu}$ is given in [56, Th. 3.9, 2.1, 4.4, Prop. 4.6] and [42].

Proof. Sufficiency is obvious.

Necessity for distributions. A characterization of surjectivity of (6.2) is given in [27, Th. 3.4]. If (6.1) is surjective then (6.2) must be surjective. Then, by [27, Th. 2.10], ker $T_{\mu} \subseteq \mathscr{D}_{(\omega)}^{\prime}(\mathbb{R})$, dim ker $T_{\mu}=\infty$, is isomorphic to $\Lambda_{\infty, \infty}(\alpha, \beta)$, where $\alpha_{j}=\left|\operatorname{Im} a_{j}\right|, \beta_{j}=\omega\left(a_{j}\right)$ and $\left(a_{j}\right)$ is a sequence with multiplicities of zeros of the Fourier transform $\hat{\mu}$ of $\mu$. By Corollary 6.4 and Corollary 2.3, ker $T_{\mu}$ is an LS-space. This completes the proof by [56, Th. 3.9 and Th. 2.1]. 
Unfortunately, it seems to be very difficult to check interpolation estimates for general operators or even differential operators with variable coefficients. In case of constant coefficients differential operators we may apply the Fundamental Principle of Ehrenpreis and Palamodov. This is the content of the next section.

\section{Evaluation of the dual interpolation estimates via Phragmén-Lindelöf type conditions}

First we need some notation and we introduce a family of Phragmén-Lindelöf type conditions. Assume from now on that $V$ is an algebraic variety, $\Omega \subseteq \mathbb{R}^{d}$ is a convex open set and $\omega$ is a weight (in the sense of definitions of spaces $\left.\mathscr{D}_{(\omega)}^{\prime}(\Omega)\right)$. We fix a convex compact exhaustion $\left(K_{N}\right)$ of $\Omega$. We define $P S H(V)$ to be the set of plurisubharmonic functions $u: V \rightarrow[-\infty, \infty[$, i.e., locally bounded from above and plurisubharmonic at regular points of $V$. We assume that the values in singular points of $V$ are defined by $u(z):=\limsup _{\xi \in V_{\text {reg }}, \xi \rightarrow z} u(\xi)$ for $z \in V_{\text {sing }}$. For a convex compact set $\emptyset \neq K \subseteq \mathbb{R}^{d}$ we define its support function

$$
h_{K}: \mathbb{R}^{d} \rightarrow \mathbb{R}, \quad h_{K}(\xi):=\sup \{\langle\xi, x\rangle: x \in K\},
$$

moreover, for any $N \in \mathbb{N}$ we define $h_{K}:=h_{K_{N}}$. Then

$$
\begin{aligned}
\operatorname{PSH}(V, N):=\{u \in \operatorname{PSH}(V): & \forall n \in \mathbb{N} \exists C_{0} \forall z \in V \\
u(z) & \left.\leq h_{N}(\operatorname{Im} z)-n \omega(z)+C_{0}\right\} .
\end{aligned}
$$

We say that the variety $V$ satisfies $\operatorname{IPL}(\Omega, \omega)$ if and only if

$$
\begin{gathered}
\forall N \exists M \geq N \quad \forall K \geq M \quad \exists n \in \mathbb{N} \forall m \in \mathbb{N} \forall \theta \in] 0,1[\exists k \in \mathbb{N}, C \\
\forall t \in \mathbb{R} \forall u \in \operatorname{PSH}(V, N) \quad(a)+(b) \Rightarrow(c),
\end{gathered}
$$

where

(a) $\forall z \in V \quad u(z) \leq h_{N}(\operatorname{Im} z)-n \omega(z)+t$;

(b) $\forall z \in V \quad u(z) \leq h_{K}(\operatorname{Im} z)-k \omega(z)$;

(c) $\forall z \in V \quad u(z) \leq h_{M}(\operatorname{Im} z)-m \omega(z)+\theta t+C$.

If all the conditions are considered only for $t \geq 0$ (or $t \leq 0$ ) we write $\operatorname{IPL}_{+}(\Omega, \omega)$ (or $\operatorname{IPL}_{-}(\Omega, \omega)$ ) and say that $V$ satisfies the IPL condition for positive (negative) $t$. Similarly, if we replace the quantifier $\forall \theta \in] 0,1[$ by

$\left.\exists \theta_{0} \in\right] 0,1[\forall \theta \in] 0, \theta_{0}$ [ we say that IPL is satisfied for small $\theta$ and denote it by $\operatorname{IPL}^{0}(\Omega, \omega)$. Analogously, if we put $\left.\exists \theta_{0} \in\right] 0,1[\forall \theta \in] \theta_{0}, 1[$ then we call the condition for big $\theta$ and denote it by $\operatorname{IPL}^{1}(\Omega, \omega)$. Of course, all the possible combinations of subscripts and superscripts are possible so we have defined nine versions of IPL. 
It is easy to observe that $\operatorname{IPL}_{+}(\Omega, \omega)$ is equivalent to $\operatorname{IPL}_{+}^{0}(\Omega, \omega)$. Similarly, the condition $\operatorname{IPL}_{-}(\Omega, \omega)$ is equivalent to $\operatorname{IPL}_{-}^{1}(\Omega, \omega)$, hence $\operatorname{IPL}(\Omega, \omega)$ is equivalent to the combination of $\operatorname{IPL}_{+}^{0}(\Omega, \omega)$ and $\operatorname{IPL}_{-}^{1}(\Omega, \omega)$.

The idea of introducing Phragmén-Lindelöf properties to our theory is clearly inspired by Hörmander's characterization of surjectivity of linear partial differential operators with constant coefficients on the space of real analytic functions [35] and the impressive research of Meise, Taylor and Vogt on the existence of linear continuous right inverses for linear partial differential operators with constant coefficients on the space of smooth functions [48] and, especially, [55]. We apply many ideas from the latter paper. In view of Problem 9.1, the guiding principle is that everything true for the right inverse should be true for the real analytic parameter dependence. Surprisingly often that is right.

Remarks. (1) Clearly we may assume $\omega(0) \geq 1$.

(2) We may remove the constant $C$ in the definitions of IPL conditions manipulating with $k$ for $t \geq 0$ or $n$ for $t \leq 0$.

Now, we formulate the main result of this section. Let us recall that for $\omega(z)=\log (2+|z|)$ the space $\mathscr{D}_{(\omega)}^{\prime}$ is just the standard space of distributions $\mathscr{D}^{\prime}$.

Theorem 7.1 Let $\Omega \subseteq \mathbb{R}^{d}$ be open convex and let $V=\{z: P(-z)=0\}$ be the zero variety of the polynomial $P$. Let $P(D): \mathscr{D}_{(\omega)}^{\prime}(\Omega) \rightarrow \mathscr{D}_{(\omega)}^{\prime}(\Omega)$ be a linear partial differential operator with constant coefficients.

(i) ker $P(D)$ has the dual interpolation estimate if and only if $V$ has $\operatorname{IPL}(\Omega, \omega)$.

(ii) ker $P(D)$ has a dual interpolation estimate for small $\theta$ (big $\theta$ ) if and only if $V$ has $\operatorname{IPL}^{0}(\Omega, \omega)\left(\operatorname{IPL}^{1}(\Omega, \omega)\right)$.

(iii) $\operatorname{ker} P(D)$ has $(P \overline{\bar{\Omega}})$ if and only if $V$ has $\operatorname{IPL}_{+}(\Omega, \omega)$ or, equivalently, $\operatorname{IPL}_{+}^{0}(\Omega, \omega)$.

(iv) ker $P(D)$ has $(P \Omega)$ if and only if $V$ has $\operatorname{IPL}_{+}^{1}(\Omega, \omega)$.

(v) ker $P(D)$ has $(P A)$ if and only if $V$ has $\operatorname{IPL}_{-}(\Omega, \omega)$ or, equivalently, $\operatorname{IPL}_{-}^{1}(\Omega, \omega)$.

(vi) if $\operatorname{ker} P(D)$ has $(P \underline{A})$ then $V$ has $\operatorname{IPL}_{-}^{0}(\Omega, \omega)$.

First, we reduce the result to irreducible varieties.

Lemma 7.2 An algebraic variety has one of the IPL properties if and only if each of its irreducible component has the same property. 
Proof. Since each plurisubharmonic function on the variety is plurisubharmonic on each irreducible component (use [38, Th. 2.9.22]) sufficiency follows.

Necessity follows from the fact that every plurisubharmonic function on an irreducible component $V_{1}$ of an algebraic variety $V$ extends to a plurisubharmonic function $\tilde{u}$ on the whole $V$ by taking $\tilde{u}(z)=-\infty$ for $z \in V \backslash V_{1}$.

We call a property $(P)$ of PLS-spaces to be a three space property of $P L S$-spaces if for every short topologically exact sequence of PLS-spaces

$$
0 \rightarrow X \rightarrow Y \rightarrow Z \rightarrow 0
$$

the space $Y$ has $(P)$ whenever $X$ and $Z$ have $(P)$.

Lemma 7.3 The following properties are the three space properties of PLSspaces: the dual interpolation estimate, the dual interpolation estimate for big $\theta$ and for small $\theta,(P \overline{\bar{\Omega}}),(P \Omega)$ and $(P A)$.

Proof. Observe that for a PLS-space $X$ we have for stable $\alpha$ :

- $X \in(P \overline{\bar{\Omega}})$ if and only if $\operatorname{Proj}^{1} X \varepsilon \Lambda_{0}^{\prime}(\alpha)=0\left(\Lambda_{0}(\alpha)\right.$ equipped with $\ell_{1}$-norms) [6, Cor. 3.8, 7.2];

- $X \in(P \Omega)$ if and only if $\operatorname{Proj}^{1} X \varepsilon \Lambda_{\infty}^{\prime}(\alpha)=0\left(\Lambda_{\infty}(\alpha)\right.$ equipped with $\ell_{1}$-norms) [6, Cor. 3.8, 7.2];

- $X \in(P A)$ if and only if $\operatorname{Proj}^{1} X \varepsilon \Lambda_{r}(\alpha)=0\left(\Lambda_{r}(\alpha)\right.$ equipped with $\ell_{\infty}$-norms) [8, Th. 4.4, 3.4];

- $X$ has the dual interpolation estimate for small $\theta$ if and only if

$$
\operatorname{Proj}^{1} X \varepsilon \mathscr{A}(\mathbb{R})=0
$$

(Cor. 6.1).

Other considered properties are combinations of the mentioned above. It suffices to show that for a fixed PLN-space $E$ the condition $\operatorname{Proj}^{1} X \varepsilon E=0$ is a three space property of PLS-spaces $X$.

Assume that $0 \rightarrow X \rightarrow Y \rightarrow Z \rightarrow 0$ is a short topologically exact sequence of PLS-spaces and $\operatorname{Proj}^{1} X \varepsilon E=\operatorname{Proj}^{1} Z \varepsilon E=0$. As in the proof of Proposition 4.5 we obtain the following exact sequence:

$$
\begin{aligned}
0 \rightarrow X \varepsilon E \rightarrow Y \varepsilon E \rightarrow Z \varepsilon E \rightarrow & \\
& \rightarrow \operatorname{Proj}^{1}(X \varepsilon E) \rightarrow \operatorname{Proj}^{1}(Y \varepsilon E) \rightarrow \operatorname{Proj}^{1}(Z \varepsilon E) \rightarrow 0
\end{aligned}
$$

and the result follows easily. 
Lemma 7.4 Let $P$ be a polynomial, $P=Q_{1} \cdots Q_{n}$ its decomposition into irreducible factors. The space $\operatorname{ker} P(D) \subseteq \mathscr{D}_{(\omega)}^{\prime}(\Omega)$ has one of the conditions $(P \overline{\bar{\Omega}}),(P \Omega),(P A)$, the dual interpolation estimate for all (big, small) $\theta$ if and only if every space ker $Q_{j}(D) \subseteq \mathscr{D}_{(\omega)}^{\prime}(\Omega)$ has the same property for $j=1, \ldots, n$.

Please observe by the proof below that necessity holds also for $(P \underline{A})$.

Proof. As in [80, Lemma 5], we have a short exact sequence

$$
0 \rightarrow \operatorname{ker}\left(P / Q_{j}\right)(D) \rightarrow \operatorname{ker} P(D) \rightarrow \operatorname{ker} Q_{j}(D) \rightarrow 0 .
$$

If ker $P(D)$ has one of the properties then $\operatorname{Proj}^{1}$ ker $P(D)=0$. By [83, Sec. 3.4.5, 3.4.6], $P(D)$ is surjective, thus all operators $Q_{j}(D)$ are surjective and $\operatorname{Proj}^{1}$ ker $Q_{j}(D)=0$. By Proposition 4.7, the sequence (7.1) is topologically exact. Hence ker $Q_{j}(D)$ is a topological quotient of ker $P(D)$ which implies necessity.

For sufficiency we apply Lemma 7.3 and inductively sequence (7.1). Let us note that if ker $Q_{j}(D)$ satisfies one of the considered conditions then $\operatorname{Proj}^{1}$ ker $Q_{j}(D)=0$ and (7.1) is topologically exact as above.

The crucial role in the proof of our result is played by the following approximation result of Meise, Taylor and Vogt [49, Th. 5.1] and Franken [26, Th. 8]. In fact, their formulation is much more complicated and based on the stronger assumption $|u(z)| \leq L|z|$. The following result gives in the simplified form the essence of the result needed later on.

Theorem 7.5 For any algebraic variety $V \subseteq \mathbb{C}^{d}$ there is a constant $C_{V}$ such that for any $u \in \operatorname{PSH}(V)$ such that

$$
|u(z)| \leq L|z|+L \quad \text { for } z \in V
$$

there exists for every point $z \in V$ an entire function $f_{z}: \mathbb{C}^{d} \rightarrow \mathbb{C}$ such that

(a) $u(z) \leq \log \left|f_{z}(\zeta)\right|+L^{2}+C_{V} \log (2+|z|)$ for some $\zeta \in V,|\zeta-z| \leq 2$;

(b) $\forall \zeta \in V \exists \zeta^{\prime} \in V \quad \log \left|f_{z}(\zeta)\right| \leq u\left(\zeta^{\prime}\right)+C_{V} \log (2+|\zeta|)$ and $\left|\zeta-\zeta^{\prime}\right| \leq 1$.

Proof. Changing constants in the proof of [26, Th. 8], we get the same result under the weaker assumption (7.2). In [26] the functions $f_{z}$ are defined only for parameters outside exceptional sets $S_{0}$ and $E$. To get them for all $z$ we need two steps. First, for $z \in S_{0}$ we find $z_{1} \in V \backslash S_{0},\left|z-z_{1}\right| \leq 1$, such that $u(z) \leq u\left(z_{1}\right)$, see [26, Prop. 7 (iv)]. Then, by [26, (8) in the proof of Th. 10] and the remarks below as well as [26, Prop. 7 (v)(c)], for $z \in V \backslash S_{0}$

$$
u(z) \leq L^{2}+u(z(\tau))
$$

for some $\tau \in B \backslash E$ and $|z-z(\tau)| \leq 1$. We define $f_{z}=f_{\tau}$, where $f_{\tau}$ is defined in [26, Th. 8]. Then we get the result by [26, Th. 8]. 
Proof of Theorem 7.1: By Lemmas 7.2 and 7.4 we may restrict our attention to irreducible polynomials $P$.

We show only the case (i). The other cases are very similar. By the Fundamental Principle for $P(D): \mathscr{D}_{(\omega)}^{\prime}(\Omega) \rightarrow \mathscr{D}_{(\omega)}^{\prime}(\Omega)$ (see [50, Lemma 3.5, 3.8], comp. [33, Sec. 4.3, 4.4]) we have

$$
(\operatorname{ker} P(D))^{\prime}=\left\{f \in H(V): \exists N \forall n \quad\|f\|_{N, n}^{*}<\infty\right\},
$$

where

$$
\|f\|_{N, n}^{*}:=\sup _{z \in V}|f(z)| \exp \left(-h_{N}(\operatorname{Im} z)+n \omega(z)\right) .
$$

Moreover, ker $P(D)=\operatorname{proj}_{N \in \mathbb{N}} X_{N}$, where $X_{N}$ are LN-spaces and

$$
X_{N}^{\prime}=\left\{f \in H(V): \forall n \in \mathbb{N} \quad\|f\|_{N, n}^{*}<\infty\right\} .
$$

The dual interpolation estimate for ker $P(D) \subseteq \mathscr{D}_{(\omega)}^{\prime}(\Omega)$ means that

$$
\begin{array}{r}
\left.\forall N^{\prime} \exists M^{\prime} \forall K^{\prime} \exists n^{\prime} \forall m^{\prime}, \theta \in\right] 0,1\left[\exists k^{\prime}, C^{\prime} \forall r>0 \forall f \in X_{N^{\prime}}^{\prime}:\right. \\
\frac{1}{r^{\theta}}\|f\|_{M^{\prime}, m^{\prime}}^{*} \leq C\left(\|f\|_{K^{\prime}, k^{\prime}}^{*}+\frac{1}{r}\|f\|_{N^{\prime}, n^{\prime}}^{*}\right) .
\end{array}
$$

Taking $\operatorname{logarithms}$ on both sides and $t=\log r$ we get $\operatorname{IPL}(\Omega, \omega)$ with the above preambule of quantifiers but only for $u$ of the form $u(z)=\log |f(z)| \in$ $\operatorname{PSH}(V, N), f \in H(V)$.

So it suffices to show that if $\operatorname{IPL}(\Omega, \omega)$ holds for such holomorphic functions then we have the same condition for $u \in \operatorname{PSH}(V)$.

Let us fix $\varepsilon>0$. By Lemma 2.2, we construct a non-quasianalytic weight $\sigma, \omega=o(\sigma)$, and a plurisubharmonic function $v: \mathbb{C}^{d} \rightarrow[-\infty, \infty[$ such that for $z \in \mathbb{C}^{d}$

$$
-D \sigma(z) \leq v(z) \leq \varepsilon|\operatorname{Im} z|-\sigma(z) .
$$

Obviously for some $\tilde{D}$ :

$$
-\tilde{D}|z|-\tilde{D} \leq v(z) \leq \varepsilon|z| .
$$

Since $\omega=o(\sigma)$, we get an increasing sequence $\left(D_{1}(p)\right)_{p \in \mathbb{N}}$ such that

$$
\forall z \in V \quad v(z) \leq \varepsilon|\operatorname{Im} z|-p \omega(z)+D_{1}(p) .
$$

Let us recall that there is a constant $A$ such that

$$
\omega(x+1) \leq A \omega(x)+A \quad \text { for all } x
$$

and we may assume that $\log (2+|z|) \leq \omega(z)$ for any $z$. 
We take arbitrary $N, B(0, \varepsilon) \subseteq K_{N}$. We take $N^{\prime}:=N$ and choose from (7.3) $M^{\prime}, M:=M^{\prime}$, then for any $K \geq M, K^{\prime}:=K$ we find $n^{\prime}$ from (7.3), $n:=A n^{\prime}+A C_{V}\left(C_{V}\right.$ comes from Theorem 7.5). Then for any $m, m^{\prime}:=A^{2} m+A^{2} C_{V}$ we choose for suitable $\theta$ a constant $k^{\prime}$ from (7.3), $k:=A k^{\prime}+A C_{V}$.

We take arbitrary $t \geq 0, u \in \operatorname{PSH}(V, N)$ such that for $z \in V$ we have:

$$
\begin{aligned}
& u(z) \leq h_{N}(\operatorname{Im} z)-n \omega(z)+t \\
& u(z) \leq h_{K}(\operatorname{Im} z)-k \omega(z) .
\end{aligned}
$$

We define

$$
\tilde{u}(z):=\max \left(u(z), v(z)-D_{1}(k)\right) .
$$

Using (7.4) and the second estimate in (7.7), let us observe that for some $L>0$ not depending on $t$ we get:

$$
|\tilde{u}(z)| \leq L|z|+L .
$$

By (7.5), $\tilde{u} \in \operatorname{PSH}(V, N)$ since $u \in \operatorname{PSH}(V, N)$. For $z \in V$ and $t \geq 0$ we get by $(7.5)$ :

$$
\begin{aligned}
\tilde{u}(z) & \leq \max \left(h_{N}(\operatorname{Im} z)-n \omega(z)+t, h_{N}(\operatorname{Im} z)-n \omega(z)+D_{1}(n)-D_{1}(k)\right) \\
& \leq h_{N}(\operatorname{Im} z)-n \omega(z)+t
\end{aligned}
$$

and

$$
\tilde{u}(z) \leq h_{K}(\operatorname{Im} z)-k \omega(z) .
$$

We apply to the function $\tilde{u}$ Theorem 7.5 , then we find entire functions $f_{z}$. Let us take $\zeta \in V$, then, by (7.8) and (7.6) for $C_{N}:=\max _{|x| \leq 1} h_{N}(x)$ :

$$
\begin{aligned}
\log \left|f_{z}(\zeta)\right| & \leq \max \left\{\tilde{u}\left(\zeta^{\prime}\right):\left|\zeta^{\prime}-\zeta\right| \leq 1\right\}+C_{V} \log (2+|\zeta|) \leq \\
& \leq h_{N}(\operatorname{Im} \zeta)+\max _{|x| \leq 1} h_{N}(x)-n \omega(|\zeta|-1)+t+C_{V} \log (2+|\zeta|) \leq \\
& \leq h_{N}(\operatorname{Im} \zeta)+C_{N}-n\left(\frac{\omega(\zeta)}{A}-1\right)+t+C_{V} \omega(\zeta) \\
& \leq h_{N}(\operatorname{Im} \zeta)-n^{\prime} \omega(\zeta)+t+C_{N}+n .
\end{aligned}
$$

On the other hand, (7.9) implies:

$$
\log \left|f_{z}(\zeta)\right| \leq h_{K}(\operatorname{Im} \zeta)-k^{\prime} \omega(\zeta)+C_{K}+k .
$$

Analogously, since $\tilde{u} \in \operatorname{PSH}(V, N)$ we get also $\log \left|f_{z}\right| \in \operatorname{PSH}(V, N)$. We apply IPL for logarithms of holomorphic functions to get

$$
\log \left|f_{z}(\zeta)\right| \leq h_{M}(\operatorname{Im} \zeta)-m^{\prime} \omega(\zeta)+\theta t+\max \left(C_{N}+n, C_{K}+k\right)+C^{\prime} .
$$


Let us take $z \in V$, then by (a) in Theorem 7.5, for $C^{\prime \prime}:=\max \left(C_{N}+n, C_{K}+\right.$ $k)+C^{\prime}+L^{2}$ and some $\zeta \in V,|\zeta-z| \leq 2$ we get

$$
\begin{aligned}
u(z) & \leq \log \left|f_{z}(\zeta)\right|+L^{2}+C_{V} \log (2+|z|) \\
& \leq h_{M}(\operatorname{Im} z)+\max _{|x| \leq 2} h_{M}(x)-m^{\prime} \omega(|z|-2)+\theta t+C^{\prime \prime}+C_{V} \log (2+|z|) \\
& \leq h_{M}(\operatorname{Im} z)+2 C_{M}-m^{\prime}\left(\frac{\omega(z)}{A^{2}}-\frac{1}{A}-1\right)+\theta t+C_{V} \omega(z)+C^{\prime \prime} \\
& \leq h_{M}(\operatorname{Im} z)-m \omega(z)+\theta t+2 C_{M}+m^{\prime} / A+m^{\prime}+C^{\prime \prime} .
\end{aligned}
$$

We have finished the proof for $t \geq 0$ with constant $C=2 C_{M}+m^{\prime} / A+m^{\prime}+C^{\prime \prime}$.

For the case $t \leq 0$ we use

$$
\begin{aligned}
& u(z) \leq h_{N}(\operatorname{Im} z)-n \omega(z), \\
& u(z) \leq h_{K}(\operatorname{Im} z)-k \omega(z)-t .
\end{aligned}
$$

The proof is similar getting

$$
u(z) \leq h_{M}(\operatorname{Im} z)-m \omega(z)+(\theta-1) t+\text { const } .
$$

We will need the following auxiliary lemma (comp. [55, Lemma 2.10]).

Lemma 7.6 Let $V$ be an algebraic variety, $A>1$. Assume that for some fixed convex compact sets $N \Subset M \Subset \mathbb{R}^{d}$ the following condition holds:

$\left.\exists n \forall m \exists \theta_{0}(m) \forall \theta \in\right] \theta_{0}(m), 1[\exists k(m, \theta), C \forall t \geq 0 \forall u \in \operatorname{PSH}(V, N)$

$$
(a)+(b) \Rightarrow(c)
$$

where

(a) $\forall z \in V \quad u(z) \leq h_{N}(\operatorname{Im} z)-n \omega(z)+t$;

(b) $\forall z \in V \quad u(z) \leq A h_{M}(\operatorname{Im} z)-k(m, \theta) \omega(z)$;

(c) $\forall z \in V \quad u(z) \leq h_{M}(\operatorname{Im} z)-m \omega(z)+\theta t+C$.

Then

$$
\begin{gathered}
\left.\forall p \geq 2 \exists n(p)>n \forall m \exists \theta_{0}(m, p) \forall \theta \in\right] \theta_{0}(m, p), 1[\exists k(m, \theta, p), C \\
\forall t \geq 0 \forall u \in \operatorname{PSH}(V, N) \quad\left(a_{p}\right)+\left(b_{p}\right) \Rightarrow\left(c_{p}\right),
\end{gathered}
$$

where

$$
\begin{array}{ll}
\left(a_{p}\right) \quad \forall z \in V & u(z) \leq h_{N}(\operatorname{Im} z)-n(p) \omega(z)+t ; \\
\left(b_{p}\right) \quad \forall z \in V & u(z) \leq A^{p} h_{M}(\operatorname{Im} z)-k(m, \theta, p) \omega(z) ; \\
\left(c_{p}\right) \quad \forall z \in V & u(z) \leq h_{M}(\operatorname{Im} z)-m \omega(z)+\theta t+C .
\end{array}
$$

An analogous result holds for other IPL-type conditions, i.e., for $t \leq 0$ or for small $\theta$.

Proof. We go by induction with respect to $p$ for $u$ applying the inductive hypothesis for $v:=u / A$. 
We show that we can manipulate with the set $\Omega$ :

Proposition 7.7 Let $\Omega \subseteq \mathbb{R}^{d}$ be an open convex set, $V$ an arbitrary algebraic variety satisfying one of the $\operatorname{IPL}(\Omega, \omega)$ conditions. Then for every $s>0$ and $a \in \mathbb{R}^{d}$ the variety $V$ satisfies the same IPL condition with the sets

$$
s \Omega=\{s x: x \in \Omega\}, \quad \Omega+a=\{x+a: x \in \Omega\}, \quad-\Omega=\{-x: x \in \Omega\} .
$$

Proof. Let us observe that for any compact set $K$

$$
h_{s K}(x)=s h_{K}(x), \quad h_{K+a}(x)=h_{K}(x)+\langle x, a\rangle, \quad h_{-K}(x)=h_{K}(-x) .
$$

Observe also that if $u(z)$ is plurisubharmonic then $u(z) / s, u(z)+\langle\operatorname{Im} z, a\rangle$, $u(-z)$ are also plurisubharmonic functions with respect to $z$. The conclusion follows easily.

Proposition 7.8 Let $\Omega_{1} \subset \Omega_{2} \subset \cdots \subset \mathbb{R}^{d}$ be an increasing sequence of open convex sets such that an algebraic variety $V$ satisfies $\operatorname{IPL}\left(\Omega_{j}, \omega\right)$ for any $j \in \mathbb{N}$. Then $V$ satisfies $\operatorname{IPL}(\Omega, \omega)$ for $\Omega=\bigcup_{j} \Omega_{j}$. The same result holds for other IPL-conditions.

Proof. By Proposition 7.7, without loss of generality we may assume that $0 \in \Omega_{1}$. Let $K_{N} \Subset \Omega$ arbitrary, then $K_{N} \Subset \Omega_{j}$ for some $j$. By $\operatorname{IPL}\left(\Omega_{j}, \omega\right)$ there is $K_{M} \Subset \Omega_{j}$ and $K_{K}, K_{M} \Subset K_{K} \Subset \Omega_{j}$ such that $0 \in \operatorname{Int} K_{M}$ and

$$
\begin{gathered}
\left.\exists n \forall m \exists \theta_{0}(m) \forall \theta \in\right] \theta_{0}(m), 1[\exists k(m, \theta), C \forall t \forall u \in \operatorname{PSH}(V, N) \\
(a)+(b) \Rightarrow(c),
\end{gathered}
$$

where

(a) $\forall z \in V \quad u(z) \leq h_{N}(\operatorname{Im} z)-n w(z)+t$,

(b) $\forall z \in V \quad u(z) \leq h_{K}(\operatorname{Im} z)-k \omega(z)$,

(c) $\forall z \in V \quad u(z) \leq h_{M}(\operatorname{Im} z)-m \omega(z)+\theta t+C$.

There is $A>1, A K_{M} \Subset K_{K}$, so that $A h_{M}(\operatorname{Im} z) \leq h_{K}(\operatorname{Im} z)$ and thus assumptions of Lemma 7.6 are satisfied. Let us take any $L \Subset \Omega$, clearly for some $p \in \mathbb{N}$ we have $A^{p} K_{M} \ni L$. The conclusion follows from Lemma 7.6 for $\operatorname{IPL}(\Omega, \omega)$.

Now, we give a reformulation of IPL conditions.

Corollary 7.9 (i) The condition $\operatorname{IPL}(\Omega, \omega)$ is equivalent to

$$
\begin{gathered}
\forall N \exists M \forall \rho>0 \exists n \forall m \forall \theta \in] 0,1[\exists k, C \forall t \in \mathbb{R} \forall u \in \operatorname{PSH}(V, N) \\
(a)+(b) \Rightarrow(c),
\end{gathered}
$$

where 
(a) $\forall z \in V \quad u(z) \leq h_{N}(\operatorname{Im} z)-n \omega(z)+t$;

(b) $\forall z \in V \quad u(z) \leq \rho|\operatorname{Im} z|-k \omega(z)$;

(c) $\forall z \in V \quad u(z) \leq h_{M}(\operatorname{Im} z)-m \omega(z)+\theta t+C$.

(ii) The condition $\operatorname{IPL}\left(\mathbb{R}^{d}, \omega\right)$ is equivalent to

$\exists A>1 \forall \rho>0 \exists n \forall m \forall \theta \in] 0,1[\exists k, C \forall t \in \mathbb{R} \forall u \in \operatorname{PSH}(V, N)$

$$
(a)+(b) \Rightarrow(c) \text {, }
$$

where $K_{N}=\bar{B}(0,1)$

(a) $\forall z \in V \quad u(z) \leq|\operatorname{Im} z|-n \omega(z)+t$;

(b) $\forall z \in V \quad u(z) \leq \rho|\operatorname{Im} z|-k \omega(z)$;

(c) $\forall z \in V \quad u(z) \leq A|\operatorname{Im} z|-m \omega(z)+\theta t+C$.

On the other hand $\operatorname{IPL}(B(0,1), \omega)$ is equivalent to

$\forall A>1 \forall \rho>0 \exists n \forall m \forall \theta \in] 0,1[\exists k, C \forall t \in \mathbb{R} \forall u \in \operatorname{PSH}(V, N)$

$$
(a)+(b) \Rightarrow(c) \text {, }
$$

where (a), (b) and (c) are the same as above.

(iii) The same results hold for other IPL-conditions.

Proof. (i): The condition implies the original $\operatorname{IPL}(\Omega, \omega)$ since

$$
h_{K}(\operatorname{Im} z) \leq \rho|\operatorname{Im} z|
$$

for some $\rho$. For the other direction, by Proposition 7.7 and its proof we may assume without loss of generality that $0 \in K_{N} \Subset K_{M} \Subset \Omega$. The result follows from Lemma 7.6. The proof of other cases is similar.

\section{Application to parameter dependence of solutions of linear partial differential operators on spaces of dis- tributions}

We consider now the case of linear partial differential operators with constant coefficients $P(D): \mathscr{D}_{(\omega)}^{\prime}(\Omega) \rightarrow \mathscr{D}_{(\omega)}^{\prime}(\Omega), \Omega \subseteq \mathbb{R}^{d}$ convex, $\omega$ a nonquasianalytic weight such that $\log (2+|z|)=o(\omega(z))$ or $\omega(z)=\log (2+|z|)$. First we observe that the only essential condition is $(P \overline{\bar{\Omega}})$ or, equivalently, the dual interpolation estimate for small $\theta$.

Proposition 8.1 Every algebraic variety has $\operatorname{IPL}^{1}(\Omega, \omega)$ and $\operatorname{IPL}_{-}(\Omega, \omega)$. 
The result follows also by [6, Cor. 8.4] and [8, Prop. 5.4] from Theorem 7.1 at least for $\omega(z)=\log (2+|z|)$ but we give below a much simpler direct proof.

Proof. By Prop. 7.7, we may assume that $0 \in K_{1} \Subset \Omega$. Since $\operatorname{IPL}_{-}(\Omega, \omega)$ is equivalent to $\operatorname{IPL}_{-}^{1}(\Omega, \omega)$ it suffices to prove $\operatorname{IPL}^{1}(\Omega, \omega)$.

We observe that for a given $N, M>N$ there is $\varepsilon>0$ such that $K_{M} \supseteq$ $K_{N}+B(0, \varepsilon)$. We choose $K$ arbitrary and find $\theta$ so close to 1 that (1$\theta) h_{K}(\operatorname{Im} z)<\varepsilon|\operatorname{Im} z|$ for any $z \in \mathbb{C}^{d}$. Moreover, we find $k$ so big that for fixed $m, n$, we have $m \leq \theta n+(1-\theta) k$. Then

$$
\begin{aligned}
\theta\left(h_{N}(\operatorname{Im} z)-n \omega(z)+t\right)+(1-\theta) & \left(h_{K}(\operatorname{Im} z)-k \omega(z)\right) \leq \\
& \leq h_{M}(\operatorname{Im} z)-m \omega(z)+\theta t .
\end{aligned}
$$

Corollary 8.2 Let $P \in \mathbb{C}\left[z_{1}, \ldots, z_{d}\right]$ be an arbitrary polynomial, $V=\{z$ : $P(-z)=0\}$ its zero variety, $\Omega \subseteq \mathbb{R}^{d}$ a convex open set. If

$$
P(D): \mathscr{D}_{(\omega)}^{\prime}(\Omega) \rightarrow \mathscr{D}_{(\omega)}^{\prime}(\Omega)
$$

is a linear partial differential operator, then ker $P(D)$ has the dual interpolation estimate for big $\theta$, i.e., $(P A)$ and $(P \Omega)$. Moreover, the following assertions are equivalent:

(a) $V$ has $\operatorname{IPL}(\Omega, \omega)$ or, it has one of the equivalent conditions: $\operatorname{IPL}^{0}(\Omega, \omega)$, $\operatorname{IPL}_{+}(\Omega, \omega), \operatorname{IPL}_{+}^{0}(\Omega, \omega)$;

(b) ker $P(D) \subseteq \mathscr{D}_{(\omega)}^{\prime}(\Omega)$ has the dual interpolation estimate for all $\theta$ (or, equiv., small $\theta)$;

(c) ker $P(D)$ has $(P \overline{\bar{\Omega}})$;

(d) $P(D): \mathscr{D}_{(\omega)}^{\prime}(\Omega, \mathscr{A}(U)) \rightarrow \mathscr{D}_{(\omega)}^{\prime}(\Omega, \mathscr{A}(U))$ is surjective;

(e) $P(D): \mathscr{D}_{(\omega)}^{\prime}\left(\Omega, \Lambda_{0}^{\prime}(\alpha)\right) \rightarrow \mathscr{D}_{(\omega)}^{\prime}\left(\Omega, \Lambda_{0}^{\prime}(\alpha)\right)$ is surjective for some (all) stable (regular) $\alpha$;

(f) $P(D): \mathscr{D}_{(\omega)}^{\prime}\left(\Omega, \mathscr{E}_{\left\{\omega_{1}\right\}}(U)\right) \rightarrow \mathscr{D}_{(\omega)}^{\prime}\left(\Omega, \mathscr{E}_{\left\{\omega_{1}\right\}}(U)\right)$ is surjective for some (all) non-quasianalytic weights $\omega_{1}$.

Remark. The first part of the Corollary 8.2 is essentially known (see [6, Cor. 8.4], [8, Prop. 5.4]) but the proof here is much simpler.

Proof. The first part and the equivalences of (a)-(c) follows from Theorem 7.1 and Proposition 8.1. (d) $\Leftrightarrow(\mathrm{b})$ : Corollary 6.4. (c) $\Leftrightarrow(\mathrm{e})$ : [6, Cor. 3.9, Th. 4.1, Th. 7.1]. (c) $\Leftrightarrow(\mathrm{f})$ : Corollary 6.5.

By [80, Th.14, Th.3], we know that the kernel of $P(D): C^{\infty}(\Omega) \rightarrow$ $C^{\infty}(\Omega)$ for convex $\Omega \subseteq \mathbb{R}^{d}, d>1$, has never $(\overline{\bar{\Omega}})$ and the same holds for arbitrary $\Omega \subseteq \mathbb{R}^{d}, d>1$, for elliptic $P$. Thus we prove part (a) of Theorem A (use Corollary 2.3, Corollary 6.4 and Corollary 8.2): 
Corollary 8.3 Let $d>1$. If $P$ is hypoelliptic and $\Omega \subseteq \mathbb{R}^{d}$ is convex or $P$ elliptic and $\Omega \subseteq \mathbb{R}^{d}$ arbitrary then

$$
P(D): \mathscr{D}^{\prime}(\Omega, \mathscr{A}(U)) \rightarrow \mathscr{D}^{\prime}(\Omega, \mathscr{A}(U))
$$

is never surjective.

Corollary 8.4 For any pseudoconvex domain $\Omega \subseteq \mathbb{C}^{d}$, $d$ arbitrary, the Cauchy-Riemann operator

$$
\bar{\partial}: \mathscr{D}^{\prime(0,0)}(\Omega, \mathscr{A}(U)) \rightarrow \operatorname{ker} \bar{\partial} \subset \mathscr{D}^{\prime(0,1)}(\Omega, \mathscr{A}(U))
$$

is never surjective.

Let us mention that Langenbruch and the author have proved recently [20, Cor. 4.9] that no elliptic $P(D): C^{\infty}(\Omega) \rightarrow C^{\infty}(\Omega)$ has real analytic parameter dependence for any open $\Omega \subseteq \mathbb{R}^{d}, d>1$.

Proof. Corollary 6.4 holds also for $\bar{\partial}: \mathscr{D}^{\prime(0, p)}(\Omega) \rightarrow$ ker $\bar{\partial} \subset \mathscr{D}^{\prime(0, p+1)}(\Omega)$ for $p \geq 0$ since $\bar{\partial}$ is open onto its image (for a simple functional analytic proof of that fact see [23, proof of Cor. 2]). It is easily seen that ker $\bar{\partial}$ has a quotient isomorphic to the space $H(U)$ of holomorphic functions over one dimensional complex open set (just take restrictions to a one dimensional complex line going through $\Omega$ ). If ker $\bar{\partial}$ has $(P \overline{\bar{\Omega}})$, equivalently, $(\overline{\bar{\Omega}})$ then $H(U)$ would have $(\overline{\bar{\Omega}})$, a contradiction with Corollary 8.3.

Remark. Since, by [65] or [68], the scalar Cauchy-Riemann complex splits from the second term on, the Cauchy-Riemann operator:

$$
\bar{\partial}: \mathscr{D}^{\prime(0, p)}(\Omega, \mathscr{A}(U)) \rightarrow \text { ker } \bar{\partial} \subset \mathscr{D}^{\prime(0, p+1)}(\Omega, \mathscr{A}(U))
$$

has even a continuous linear right inverse (which implies surjectivity) for $p \geq 1$.

The next result (a part of Theorem B) is a surprisingly easy consequence of the whole previous theory and it shows that the real analytic parameter dependence is less restrictive if considered for distributions on the whole plane.

Theorem 8.5 Let $P \in \mathbb{C}\left[z_{1}, \ldots, z_{d}\right]$ be an arbitrary polynomial and $\Omega \subseteq \mathbb{R}^{d}$ an open convex set, $\omega$ a suitable weight. If

$$
P(D): \mathscr{D}_{(\omega)}^{\prime}(\Omega, \mathscr{A}(U)) \rightarrow \mathscr{D}_{(\omega)}^{\prime}(\Omega, \mathscr{A}(U))
$$

is surjective then

$$
P(D): \mathscr{D}_{(\omega)}^{\prime}\left(\mathbb{R}^{d}, \mathscr{A}(U)\right) \rightarrow \mathscr{D}_{(\omega)}^{\prime}\left(\mathbb{R}^{d}, \mathscr{A}(U)\right)
$$

is surjective as well. 
Moreover, if $v$ is an arbitrary vector such that the hyperplane

$$
H_{c}(v):=\{x:\langle x, v\rangle=c\}
$$

is tangent to the boundary of $\Omega$ at $x_{0}$ (the point of smoothness of the boundary) then

$$
P(D): \mathscr{D}_{(\omega)}^{\prime}\left(H_{+}(v), \mathscr{A}(U)\right) \rightarrow \mathscr{D}_{(\omega)}^{\prime}\left(H_{+}(v), \mathscr{A}(U)\right)
$$

is surjective as well, for $H_{+}(v):=\{x:\langle x, v\rangle>0\}$.

Proof. By Corollary 8.2, it suffices to prove that $\operatorname{IPL}_{+}^{0}(\Omega, \omega)$ implies $\mathrm{IPL}_{+}^{0}\left(\mathbb{R}^{d}, \omega\right)$ and $\operatorname{IPL}_{+}^{0}\left(H_{+}(v), \omega\right)$. This follows by Prop. 7.7 and Prop 7.8 since if $0 \in \Omega$ then $\mathbb{R}^{d}=\bigcup_{n>0} n \Omega$ and if $x_{0}=0$ then $H_{+}(v)=\bigcup_{n>0} n \Omega$.

The second part of Theorem B, one of the main and most surprising results of the paper requires a longer proof.

Theorem 8.6 Let $P \in \mathbb{C}\left[z_{1}, \ldots, z_{d}\right]$ be an arbitrary polynomial and $P_{p}$ its principal part, $\Omega \subseteq \mathbb{R}^{d}$ open convex set, $\omega$ arbitrary weight. If

$$
P(D): \mathscr{D}_{(\omega)}^{\prime}(\Omega, \mathscr{A}(U)) \rightarrow \mathscr{D}_{(\omega)}^{\prime}(\Omega, \mathscr{A}(U))
$$

is a surjective operator then

$$
P_{p}(D): \mathscr{D}_{(\omega)}^{\prime}(\Omega) \rightarrow \mathscr{D}_{(\omega)}^{\prime}(\Omega)
$$

has a linear continuous right inverse, in particular,

$$
P_{p}(D): \mathscr{D}_{(\omega)}^{\prime}(\Omega, \mathscr{A}(U)) \rightarrow \mathscr{D}_{(\omega)}^{\prime}(\Omega, \mathscr{A}(U))
$$

is surjective and has a linear continuous right inverse.

It implies that real analytic parameter dependence is a very restrictive property.

Corollary 8.7 Let $P \in \mathbb{C}\left[z_{1}, \ldots, z_{d}\right]$ be an arbitrary polynomial and $P_{p}$ its principal part, $\Omega \subseteq \mathbb{R}^{d}$ bounded open convex set with $C^{1}$-boundary, $\omega$ arbitrary weight. If

$$
P(D): \mathscr{D}_{(\omega)}^{\prime}(\Omega, \mathscr{A}(U)) \rightarrow \mathscr{D}_{(\omega)}^{\prime}(\Omega, \mathscr{A}(U))
$$

is a surjective operator then $P_{p}$ is proportional to a product of real linear forms.

Proof. Apply Theorem 8.6 and [48, Th. 3.8], [55, Th.3.3]. 
Proof of Theorem 8.6. By Corollary 8.2, the zero variety $V:=\{z:$ $P(-z)=0\}$ of $P$ has $\operatorname{IPL}_{+}^{0}(\Omega, \omega)$. Let $V_{p}$ denote the zero variety of $P_{p}$. By $\left[52\right.$, Th. 5.5] and [55, Th. 3.3], if $V_{p}$ has the so-called condition $\operatorname{HPL}(\Omega, l o c)$ at zero then

$$
P_{p}: \mathscr{D}_{(\omega)}^{\prime}(\Omega) \rightarrow \mathscr{D}_{(\omega)}^{\prime}(\Omega)
$$

has a continuous linear right inverse (and, of course, the same holds for the vector valued version of $\left.P_{p}(D)\right)$. So it suffices to show that $\operatorname{IPL}_{+}^{0}(\Omega, \omega)$ for $V$ implies $\operatorname{HPL}(\Omega, l o c)$ at zero for $V_{p}$, i.e., that there are open sets $U_{1} \subseteq U_{2} \subseteq$ $U_{3} \Subset \mathbb{C}^{d}, 0 \in U_{1}$ such that

$$
\forall N \exists M, \delta>0 \forall u \in \operatorname{PSH}\left(U_{3} \cap V\right) \quad(\alpha)+(\beta) \Rightarrow(\gamma),
$$

where

$(\alpha) u(z) \leq h_{N}(\operatorname{Im} z)+\delta$ for $z \in U_{3} \cap V$;

( $\beta) u(z) \leq 0$ for $z \in U_{2} \cap \mathbb{R}^{d} \cap V$;

$(\gamma) u(z) \leq h_{M}(\operatorname{Im} z)$ for $z \in U_{1} \cap V$.

We will use some ideas of [55, Th. 4.1]. Let us define a maximal function

$$
\begin{aligned}
& U_{E}(z ; h, V, D):= \\
& \quad \sup \{u(z): u \in \operatorname{PSH}(V \cap D), u \leq h \text { on } D \cap V, u \leq 0 \text { on } E \cap V\},
\end{aligned}
$$

where $D$ is a domain in $\mathbb{C}^{d}, h$ a function on $D, E \subseteq D$. For $0<\varepsilon<1$ we define

$$
\begin{aligned}
K_{\varepsilon} & :=\left\{z \in \mathbb{C}^{d}:|\operatorname{Im} z| \leq \varepsilon|z| \text { or }|z| \leq \varepsilon\right\} \cap \bar{B}(0,2), \\
E & =\mathbb{R}^{d} \cap \bar{B}(0,2)=\bigcap_{\varepsilon>0} K_{\varepsilon}, \\
V_{j} & :=\{z / j: z \in V\} \quad \text { for } j \in \mathbb{N} .
\end{aligned}
$$

Our aim is to estimate for some $M_{1}, \delta>0$ and all $z \in B(0,1) \cap V$ :

$$
U_{E}\left(z, h_{N} \circ \operatorname{Im}+\delta, V, B(0,3)\right) \leq h_{M_{1}}(\operatorname{Im} z)
$$

which implies $\operatorname{HPL}(\Omega, l o c)$ for $V$ at zero.

Let us take any $N$, without loss of generality (see Prop. 7.7) we may assume that $K_{N} \supset B(0,1)$. We find $N_{1}>N$ such that

$$
h_{N_{1}}(\operatorname{Im} z) \geq h_{N}(\operatorname{Im} z)+\eta|\operatorname{Im} z|>|\operatorname{Im} z|
$$

for suitable $\eta, 0<\eta<1$, small enough. 
Choose $\delta>0, \delta<\eta / 2$. By $\operatorname{IPL}_{+}^{0}(\Omega, \omega)$ and Corollary 7.9 , there is $M$ such that for each $\rho>0$ if $\varphi \in \operatorname{PSH}\left(V, N_{1}\right)$ and for all $z \in V$

$$
\begin{aligned}
& \varphi(z) \leq h_{N_{1}}(\operatorname{Im} z)-n \omega(z)+t \\
& \varphi(z) \leq \rho|\operatorname{Im} z|-k \omega(z)
\end{aligned}
$$

then for all $z \in V$

$$
\varphi(z) \leq h_{M}(\operatorname{Im} z)-m \omega(z)+\theta t+C_{\theta, m, \rho} .
$$

Choose an arbitrary $\varepsilon, 0<\varepsilon<1$, $\rho_{1}$ so large that

$$
h_{N_{1}}(\operatorname{Im} z) \leq \rho_{1}|\operatorname{Im} z|, \quad \rho:=\rho(\varepsilon):=1+\rho_{1}+\delta \varepsilon^{-2}+\eta \varepsilon^{-2} .
$$

Let us fix $j \in \mathbb{N}$. Take $u \in \operatorname{PSH}\left(V_{j} \cap B(0,3)\right)$ satisfying

$$
\begin{aligned}
& u(z) \leq h_{N}(\operatorname{Im} z)+\delta \\
& \text { for } V_{j} \cap B(0,3) \text {, } \\
& u(z) \leq 0 \\
& \text { for } z \in K_{\varepsilon} \cap V_{j} \text {. }
\end{aligned}
$$

Take an arbitrary fixed $w=\left(w_{1}, \ldots, w_{d}\right) \in V_{j} \cap B(0,1)$. Define for $z=$ $\left(z_{1}, \ldots, z_{d}\right) \in \mathbb{C}^{d}$

$$
\psi: V \cap\left\{z \in \mathbb{C}^{d}:|z-\operatorname{Re}(j w)| \leq j\right\} \rightarrow[-1, \infty[
$$

by

$$
\psi(z):=\max \left\{j u(z / j)-\frac{\eta j}{2} \operatorname{Re}\left[\sum_{p=1}^{d}\left(\frac{z_{p}}{j}-\operatorname{Re} w_{p}\right)^{2}\right], h_{N_{1}}(\operatorname{Im} z)\right\} .
$$

We extend $\psi$ to a plurisubharmonic function on the whole $V$. Indeed, observe that for

$$
z \in V, \quad|z-\operatorname{Re}(j w)|=j, \quad \text { i.e., }|\operatorname{Im} z| \leq j
$$

we get

$$
\begin{aligned}
j u(z / j)-\frac{\eta j}{2} \operatorname{Re} & {\left[\sum_{p=1}^{d}\left(\frac{z_{p}}{j}-\operatorname{Re} w_{p}\right)^{2}\right] \leq } \\
& \leq j h_{N}\left(\frac{\operatorname{Im} z}{j}\right)+j \delta+\eta j\left(\frac{|\operatorname{Im} z|}{j}-\frac{1}{2}\right) \leq h_{N_{1}}(\operatorname{Im} z) .
\end{aligned}
$$

Hence, we can extend $\psi$ by taking

$$
\psi(z):=h_{N_{1}}(\operatorname{Im} z) \quad \text { for }|z-\operatorname{Re}(j w)| \geq j .
$$

For $|z-\operatorname{Re}(j w)| \leq j$ we have $\left|\frac{\operatorname{Im} z}{j}\right| \leq 1$ and

$$
\begin{aligned}
\psi(z) & \leq \max \left(h_{N}(\operatorname{Im} z)+j \delta+\eta j\left|\frac{\operatorname{Im} z}{j}\right|^{2}, h_{N_{1}}(\operatorname{Im} z)\right) \\
& \leq h_{N_{1}}(\operatorname{Im} z)+j(\delta+\eta) .
\end{aligned}
$$


Combining the two inequalities above we get

$$
\psi(z) \leq h_{N_{1}}(\operatorname{Im} z)+j(\delta+\eta) \quad \text { for } z \in V .
$$

We will prove now that

$$
\psi(z) \leq \rho|\operatorname{Im} z| \quad \text { for } z \in V .
$$

We consider separately three cases, $z \in V$ :

$1^{\circ}|z-\operatorname{Re}(j w)| \geq j ;$

$2^{\circ}|z-\operatorname{Re}(j w)|<j, z / j \in K_{\varepsilon} ;$

$3^{\circ}|z-\operatorname{Re}(j w)|<j, z / j \notin K_{\varepsilon}$.

$1^{\circ}$. Clearly $\psi(z) \leq h_{N_{1}}(\operatorname{Im} z) \leq \rho_{1}|\operatorname{Im} z| \leq \rho|\operatorname{Im} z|$.

$2^{\circ}$. Since $|\operatorname{Im} z / j| \leq 1$ and $z / j \in K_{\varepsilon}, u(z / j) \leq 0$ and

$$
\begin{aligned}
\psi(z) & \leq \max \left\{-\frac{\eta j}{2} \operatorname{Re}\left[\sum_{p=1}^{d}\left(\frac{z_{p}}{j}-\operatorname{Re} w_{p}\right)^{2}\right], h_{N_{1}}(\operatorname{Im} z)\right\} \\
& \leq \max \left\{\eta|\operatorname{Im} z|, h_{N_{1}}(\operatorname{Im} z)\right\} \leq \rho|\operatorname{Im} z| .
\end{aligned}
$$

$3^{\circ}$. As in (8.2) we observe that $\psi(z) \leq h_{N_{1}}(\operatorname{Im} z)+j(\delta+\eta)$. Since $j \leq|z| / \varepsilon$ and $|z| \leq|\operatorname{Im} z| / \varepsilon$ we get

$$
\psi(z) \leq h_{N_{1}}(\operatorname{Im} z)+\frac{(\delta+\eta)|\operatorname{Im} z|}{\varepsilon^{2}} \leq \rho|\operatorname{Im} z| .
$$

We have proved (8.4) for all $z \in V$.

Assume that $0<\gamma<1,(1+\gamma) K_{M} \Subset K_{M_{1}} \Subset \Omega$. By Lemma 2.2, there is a non-quasianalytic weight $\sigma$ such that $\omega=o(\sigma), \omega \leq \sigma$ and there is a plurisubharmonic function $\varphi_{k}$ such that

$$
-C_{k} \sigma(z) \leq \varphi_{k}(z) \leq \gamma|\operatorname{Im} z|-k \sigma(z)
$$

for $k \in \mathbb{N}$ and some $C_{k}>0$. This is the first place where we have to assume that $\omega$ is a non-quasianalytic weight.

We define a plurisubharmonic function $\tilde{\psi}_{k}$ on $V$ ( $k$ depends on $\theta$ as in $\left.\operatorname{IPL}_{+}^{0}(\Omega, \omega)\right)$ :

$$
\tilde{\psi}_{k}(z):=\frac{\psi(z)+\varphi_{2 k}(z)}{1+\gamma} .
$$

From (8.3) it follows for $z \in V$

$$
\begin{aligned}
\tilde{\psi}_{k}(z) & \leq \frac{1}{1+\gamma} \cdot\left(h_{N_{1}}(\operatorname{Im} z)+j(\delta+\eta)+\gamma|\operatorname{Im} z|-2 k \sigma(z)\right) \\
& \leq h_{N_{1}}(\operatorname{Im} z)+j(\delta+\eta)-k \omega(z)
\end{aligned}
$$


and analogously $\tilde{\psi}_{k} \in \operatorname{PSH}\left(V, N_{1}\right)$. Similarly, by (8.4), it follows for $z \in V$

$$
\tilde{\psi}_{k}(z) \leq \frac{1}{1+\gamma} \cdot(\rho|\operatorname{Im} z|+\gamma|\operatorname{Im} z|-2 k \sigma(z)) \leq \rho|\operatorname{Im} z|-k \omega(z) .
$$

By (8.1), i.e., by $\operatorname{IPL}_{+}^{0}(\Omega, \omega)$,

$$
\tilde{\psi}_{k}(z) \leq h_{M}(\operatorname{Im} z)-m \omega(z)+\theta j(\delta+\eta)+C_{\theta, m, \rho} \quad \text { for } z \in V .
$$

By the definition of $\tilde{\psi}_{k}$ we get for $z \in V$

$$
\psi(z) \leq(1+\gamma)\left(h_{M}(\operatorname{Im} z)-m \omega(z)+\theta j(\delta+\eta)+C_{\theta, m, \rho}\right)+C_{2 k} \sigma(z) .
$$

Let us take $z=j w \in V$, then $|z-\operatorname{Re}(j w)|=|\operatorname{Im}(j w)| \leq j|w| \leq j$ and $j u(z / j) \leq \psi(z)$. Hence

$$
\begin{aligned}
j u(w) & =j u(z / j) \\
& \leq h_{M_{1}}(\operatorname{Im}(j w))-(1+\gamma)\left(m \omega(j w)-\theta j(\delta+\eta)-C_{\theta, m, \rho}\right)+C_{2 k} \sigma(j w) .
\end{aligned}
$$

We get for all $w \in V_{j} \cap B(0,1)$

$$
\begin{aligned}
& U_{K_{\varepsilon}}\left(w, h_{N} \circ \operatorname{Im}+\delta, V_{j}, B(0,3)\right) \leq \\
& \quad \leq h_{M_{1}}(\operatorname{Im} w)-(1+\gamma)\left(m w(j w) / j-\theta(\delta+\eta)-C_{\theta, m, \rho} / j\right)+C_{2 k} \sigma(j w) / j .
\end{aligned}
$$

Since $V_{j}$ converge to $V_{p}$ in the sense of [51, Def. 4.3], by [51, Th. 4.4], we get as $j \rightarrow \infty$ for $z \in V \cap B(0,1)$ the following inequality for regular points $z \in V \cap B(0,1)$

$$
U_{K_{\varepsilon}}\left(z, h_{N} \circ \operatorname{Im}+\delta, V, B(0,3)\right) \leq h_{M_{1}}(\operatorname{Im} z)+\theta(1+\gamma)(\delta+\eta)
$$

since $\omega(t) / t, \sigma(t) / t \rightarrow 0$ as $t \rightarrow \infty$ (here non-quasianaliticity of $\omega$ and $\sigma$ is used). On singular points we define plurisubharmonic functions via upper limits, so the same inequality holds for singular points as well. Since this is true for any $\theta \in] 0, \theta_{0}[$ we get for $z \in V \cap B(0,1)$

$$
U_{K_{\varepsilon}}\left(z, h_{N} \circ \operatorname{Im}+\delta, V, B(0,3)\right) \leq h_{M_{1}}(\operatorname{Im} z) .
$$

By [51, Prop. 4.2],

$$
U_{E}\left(z, h_{N} \circ \operatorname{Im}+\delta, V, B(0,3)\right) \leq h_{M_{1}}(\operatorname{Im} z),
$$

since $E=\bigcap_{\varepsilon>0} K_{\varepsilon}$.

Now, we show that the real analytic parameter dependence of solutions implies (is equivalent) to the existence of a linear continuous right inverse in many cases. We start with homogeneous polynomials and part (d) of Theorem A. 
Theorem 8.8 Let $P \in \mathbb{C}\left[z_{1}, \ldots, z_{d}\right]$ be an arbitrary homogenenous polynomial, $\Omega \subseteq \mathbb{R}^{d}$ an open convex set, $\omega$ an arbitrary non-quasianalytic weight. The operator

$$
P(D): \mathscr{D}_{(\omega)}^{\prime}(\Omega, \mathscr{A}(U)) \rightarrow \mathscr{D}_{(\omega)}^{\prime}(\Omega, \mathscr{A}(U))
$$

is surjective if and only if

$$
P(D): \mathscr{D}_{(\omega)}^{\prime}(\Omega) \rightarrow \mathscr{D}_{(\omega)}^{\prime}(\Omega) \quad\left(\text { or, equivalently, } P(D): C^{\infty}(\Omega) \rightarrow C^{\infty}(\Omega)\right)
$$

has a continuous linear right inverse. In particular, for bounded convex $\Omega$ with $C^{1}$-boundary it holds if and only if $P$ is proportional to a product of real linear forms.

Remarks. (1) This result shows a dramatic difference between real analytic and holomorphic or smooth dependence - the latter two holds always, see [8].

(2) By [48], [52, Th.5.6], for homogeneous $P$ the existence of a right inverse on $\mathscr{D}_{(\omega)}^{\prime}(\Omega)$ or $\mathscr{E}_{\{\omega\}}(\Omega), \omega$ non-quasianalytic, does not depend on $\omega$ and it is equivalent to the existence of a right inverse on $C^{\infty}(\Omega)$.

In fact, the above result can be reformulated as a new elegant characterization of the existence of a continuous linear right inverse.

Proof of Theorem 8.8: The result follows immediately from Theorem 8.6, [48, Th. 3.8] and [55, Th. 3.3]. One can also give a simpler direct proof - we will sketch it. By [52, Th. 5.5], it suffices to show for homogeneous varieties $V$ that $\operatorname{IPL}_{+}^{0}(\Omega, \omega)$ implies $\operatorname{PL}(\Omega, \omega)$ (see [55, Def. 2.5, Prop. 2.8]), i.e.,

$$
\forall N \exists M \geq N \forall K \geq M \exists B>0 \forall u \in \operatorname{PSH}(V) \quad(\alpha)+(\beta) \Rightarrow(\gamma),
$$

where

$(\alpha) u(z) \leq h_{N}(\operatorname{Im} z)+C \quad$ for some $C$ and any $z \in V$,

( $\beta) u(z) \leq h_{K}(\operatorname{Im} z) \quad$ for any $z \in V$,

$(\gamma) u(z) \leq h_{M}(\operatorname{Im} z)+B \omega(z) \quad$ for any $z \in V$.

Assume that $K_{N} \supseteq B(0,1)$ (use Proposition 7.7) and $u \in \operatorname{PSH}(V)$ satisfies $(\alpha)$ and $(\beta)$. Let $\varepsilon, 0<\varepsilon<1$, be so small that $(1+\varepsilon) K_{M} \Subset K_{M_{1}} \Subset \Omega$. By Lemma 2.2, we find a non-quasianalytic weight $\sigma \geq \omega, \omega=o(\sigma)$, and $\varphi_{k} \in \operatorname{PSH}\left(\mathbb{C}^{d}\right)$ such that

$$
-C_{k} \sigma(z) \leq \varphi_{k}(z) \leq \varepsilon|\operatorname{Im} z|-2 k \sigma(z) .
$$


We apply $\operatorname{IPL}_{+}^{0}(\Omega, \omega)$ to

$$
v_{r, k}(z):=\frac{(1 / r) u(r z)+\varphi_{k}(z)}{1+\varepsilon} \quad \text { for } r>0
$$

and then taking $r \rightarrow 0$ and $\theta \rightarrow 0$ we get

$$
u(\zeta) \leq h_{M_{1}}(\operatorname{Im} \zeta)
$$

since $\sigma(t) / t, \omega(t) / t \rightarrow 0$ as $t \rightarrow \infty$.

Before we consider further cases we need the following lemma, a strengthening of [55, Lemma 4.7].

Lemma 8.9 Assume that $V$ is an algebraic variety in $\mathbb{C}^{d}$ that satisfies $\mathrm{IPL}_{+}^{0}\left(\mathbb{R}^{d}, \omega\right)$ in the form described in Corollary 7.9. Let $M>0$, then there is a constant $C$ such that

$$
|\operatorname{Im} w| \leq C \omega(w) .
$$

for any $w=\left(w_{1}, \ldots, w_{d}\right) \in V$ satisfying

$$
|\operatorname{Im} w| \leq M|\operatorname{Im} z|
$$

for all $z$ in the connected component $V_{w}$ of $w$ in the set

$$
V \cap\left\{z \in \mathbb{C}^{d}:|z-w|<s|\operatorname{Im} w|\right\}
$$

for some $s \geq 4 A+4$.

Proof. Assume that there is a sequence $\left(w_{j}\right)_{j \in \mathbb{N}}$ satisfying assumptions of Lemma for fixed $M$ such that $\frac{\left|\operatorname{Im} w_{j}\right|}{\omega\left(w_{j}\right)} \rightarrow \infty$. Since $\omega \geq 1,\left|w_{j}\right| \rightarrow \infty$ and then we can construct a function $h:\left[0, \infty\left[\rightarrow\left[0, \infty\left[\right.\right.\right.\right.$ such that $h\left(\left|w_{j}\right|\right)=$ $\left|\operatorname{Im} w_{j}\right|$ for infinitely many $j$ and $\omega=o(h)$. By Lemma 2.2 there is a nonquasianalytic weight $\sigma \geq \omega, \omega=o(\sigma), \sigma=o(h)$. Clearly, then $\frac{\left|\operatorname{Im} w_{j}\right|}{\sigma\left(w_{j}\right)} \rightarrow \infty$. Summarizing, it suffices to prove

$$
|\operatorname{Im} w| \leq C \sigma(w) .
$$

We define $u: V_{w} \rightarrow \mathbb{R}$ for $z=\left(z_{1}, \ldots, z_{d}\right)$ by

$$
u(z):=\max \left\{(2 A+1)|\operatorname{Im} w|-\frac{1}{2 s|\operatorname{Im} w|} \operatorname{Re}\left[\sum_{p=1}^{d}\left(z_{p}-w_{p}\right)^{2}\right],|\operatorname{Im} z|\right\} .
$$

For $z \in V$ with $|z-w|=s|\operatorname{Im} w|$ we get

$$
\begin{aligned}
(2 A+1)|\operatorname{Im} w| & -\frac{s|\operatorname{Im} w|}{2} \operatorname{Re}\left[\sum_{p=1}^{d}\left(\frac{z_{p}-w_{p}}{s|\operatorname{Im} w|}\right)^{2}\right] \leq \\
& \leq(2 A+1)|\operatorname{Im} w|+s|\operatorname{Im} w|\left(\frac{|\operatorname{Im}(z-w)|}{s|\operatorname{Im} w|}-\frac{1}{2}\right) \leq|\operatorname{Im} z| .
\end{aligned}
$$


Hence we can extend $u$ to a plurisubharmonic function on $V$ defining $u(z)=|\operatorname{Im} z|$ outside $V_{w}$. Take $\rho=(A+1) M+1, m$ arbitrary, $\theta \leq$ $\frac{1}{2(2+2 A)}$ and find $k$ from $\operatorname{IPL}_{+}^{0}\left(\mathbb{R}^{d}, \omega\right)$. Then we define, by Lemma 2.2 , a plurisubharmonic function $\varphi$ on $\mathbb{C}^{d}$ such that

$$
-C \sigma(z) \leq \varphi(z) \leq|\operatorname{Im} z|-2 k \sigma(z) .
$$

We take a function

$$
v(z):=(u(z)+\varphi(z)) / 2 .
$$

Clearly, outside $V_{w}$ we have

$$
v(z) \leq|\operatorname{Im} z|-k \sigma(z)
$$

thus $v \in \operatorname{PSH}(V, \bar{B}(0,1))$. Moreover since on $V_{w}$ we have $|\operatorname{Im} w| \leq M|\operatorname{Im} z|$ thus

$$
\begin{aligned}
& (2 A+1)|\operatorname{Im} w|-\frac{s|\operatorname{Im} w|}{2} \operatorname{Re}\left[\sum_{p=1}^{d}\left(\frac{z_{p}-w_{p}}{s|\operatorname{Im} w|}\right)^{2}\right] \leq \\
& \quad \leq(2 A+1)|\operatorname{Im} w|+|\operatorname{Im} z|+|\operatorname{Im} w| \leq((2 A+2) M+1)|\operatorname{Im} z|
\end{aligned}
$$

Clearly

$$
v(z) \leq((A+1) M+1)|\operatorname{Im} z|-k \sigma(z) \leq \rho|\operatorname{Im} z|-k \omega(z) .
$$

On the other hand, by (8.5),

$$
v(z) \leq|\operatorname{Im} z|+(A+1)|\operatorname{Im} w|-k \sigma(z) \leq|\operatorname{Im} z|+(A+1)|\operatorname{Im} w|-n w(z) .
$$

Taking $t=(A+1)|\operatorname{Im} w|$ we get by $\operatorname{IPL}_{+}^{0}\left(\mathbb{R}^{d}, \omega\right)$

$$
v(z) \leq A|\operatorname{Im} z|-m w(z)+(1 / 4)|\operatorname{Im} w|,
$$

therefore

$$
u(z) \leq 2 A|\operatorname{Im} z|-2 m w(z)+(1 / 2)|\operatorname{Im} w|+C \sigma(z) .
$$

Applying the above inequality for $z=w$ we get $|\operatorname{Im} w| \leq 2 C \sigma(w)$.

Proposition 8.10 Let $d \in \mathbb{N}, P \in \mathbb{C}\left[z_{1}, \ldots, z_{d}, z_{d+1}, z_{d+2}\right]$ be a polynomial of the form

$$
P\left(z_{1}, \ldots, z_{d+2}\right)=\sum_{j=1}^{d} \varepsilon_{j} z_{j}^{2}+\lambda z_{d+1}+\mu z_{d+2},
$$

where $\varepsilon_{j}= \pm 1, \lambda \in \mathbb{C} \backslash \mathbb{R}, \mu \in \mathbb{R}$. Then the zero variety $V(P)$ has no $\operatorname{IPL}_{+}^{0}\left(\mathbb{R}^{d+2}, \omega\right)$ for any non-quasianalytic weight $\omega, \omega(t)=o\left(t^{(1 / 2)}\right)$. 
Proof. We take any non-quasianalytic weight $\sigma, \omega=o(\sigma), \sigma \geq \omega, \sigma(t)=$ $o\left(t^{1 / 2}\right)$, comp. Lemma 2.2. Exactly as in the proof of [53, Lemma 4] we prove the inequality (2) there using Lemma 8.9 instead of [55, Lemma 4.7] and we get

$$
R \sin \frac{\delta}{2} \leq C \omega\left(R^{2}\right) \quad \text { for } R \geq R_{0},
$$

a contradiction.

We are ready to solve the case of polynomials of order two and we prove Theorem A (c).

Theorem 8.11 If $P \in \mathbb{C}\left[z_{1}, \ldots, z_{d}\right]$ is an arbitrary polynomial of order two such that there exists an open convex set $\Omega \subseteq \mathbb{R}^{d}$ for which

$$
P(D): \mathscr{D}^{\prime}(\Omega, \mathscr{A}(U)) \rightarrow \mathscr{D}^{\prime}(\Omega, \mathscr{A}(U))
$$

is surjective then $P(D)$ is as in Theorem $A(c)$.

It is proved in [53, Th. 2] for $P$ of order two that $P(D): \mathscr{D}^{\prime}\left(\mathbb{R}^{d}\right) \rightarrow \mathscr{D}^{\prime}\left(\mathbb{R}^{d}\right)$ has a continuous linear right inverse if and only if the necessary condition above holds:

Corollary 8.12 If $P \in \mathbb{C}\left[z_{1}, \ldots, z_{d}\right]$ is an arbitrary polynomial of second order then

$$
P(D): \mathscr{D}^{\prime}\left(\mathbb{R}^{d}, \mathscr{A}(U)\right) \rightarrow \mathscr{D}^{\prime}\left(\mathbb{R}^{d}, \mathscr{A}(U)\right)
$$

is surjective if and only if

$$
P(D): \mathscr{D}^{\prime}\left(\mathbb{R}^{d}\right) \rightarrow \mathscr{D}^{\prime}\left(\mathbb{R}^{d}\right)
$$

has a continuous linear right inverse.

Proof of Theorem 8.11. By Theorem 8.5 and Theorem 8.6, if

$$
P(D): \mathscr{D}^{\prime}(\Omega, \mathscr{A}(U)) \rightarrow \mathscr{D}^{\prime}(\Omega, \mathscr{A}(U))
$$

is surjective then

$$
P(D): \mathscr{D}^{\prime}\left(\mathbb{R}^{d}, \mathscr{A}(U)\right) \rightarrow \mathscr{D}^{\prime}\left(\mathbb{R}^{d}, \mathscr{A}(U)\right)
$$

is surjective and

$$
P_{p}(D): \mathscr{D}^{\prime}\left(\mathbb{R}^{d}\right) \rightarrow \mathscr{D}^{\prime}\left(\mathbb{R}^{d}\right)
$$

has a continuous linear right inverse.

By [53, Lemma 2], there is $\lambda \in \mathbb{C}$ such that $\lambda P_{p}$ is real. As in the proof of $[53$, Lemma $3(i)]$, the real analytic parameter dependence on $\mathbb{R}^{d}$ does not change if we make either a real linear invertible change of variables or a complex translation of variables in the symbol $P$. By [53, Lemma 2], doing such changes of variables we may assume that $P$ is one of the forms:

(i) $P(z)=\sum_{j=1}^{r} z_{j}^{2}-\sum_{j=r+1}^{s} z_{j}^{2}+c, c \in \mathbb{C}$; 
(ii) $P(z)=\sum_{j=1}^{r} z_{j}^{2}-\sum_{j=r+1}^{s} z_{j}^{2}+\lambda z_{s+1}, \lambda \in \mathbb{C} \backslash\{0\}$;

(iii) $P(z)=\sum_{j=1}^{r} z_{j}^{2}-\sum_{j=r+1}^{s} z_{j}^{2}+i z_{s+1}+z_{s+2}$.

By Proposition 8.10, $P$ cannot be of the form (iii) and in the form (ii) $\lambda$ must be real. Since

$$
P_{p}(z)=\sum_{j=1}^{r} z_{j}^{2}-\sum_{j=r+1}^{s} z_{j}^{2}
$$

and $P_{p}(D)$ has a continuous linear right inverse, then by [53, Prop. 2], either $r=s=1$ or $s>r$. If in case (ii) $r=s=1$ then

$$
P(z)=z_{1}^{2}+\lambda z_{2} \quad \lambda \in \mathbb{R} .
$$

Of course, if $P(D)$ has the real analytic parameter dependence on $\mathbb{R}^{d}, d>2$, then it has such a dependence for $\mathbb{R}^{2}$ and the zero variety $V(P) \subseteq \mathbb{C}^{2}$ has $\mathrm{IPL}_{+}^{0}\left(\mathbb{R}^{2}, \log (2+|\cdot|)\right)$, see Cor. 8.2.

We will show by Lemma 8.9 that this is impossible. Take a point

$$
z_{r}:=\left(-i r,-r^{2} / \lambda\right) \in V(P) \quad \text { for } r \in \mathbb{R} .
$$

Let us fix $s>0$, then for $\left(-z, z^{2} / \lambda\right) \in V(P)$ if we have

$$
\left|\left(-z, z^{2} / \lambda\right)-\left(-i r,-r^{2} / \lambda\right)\right| \leq s\left|\operatorname{Im} z_{r}\right|=s|r|,
$$

then $\left|z^{2}+r^{2}\right| \leq \lambda s|r|$. Hence for $r$ big enough and suitable small $\delta$ :

$$
|z| \geq r / 2, \quad \operatorname{Arg} z \in[\pi / 2-\delta / 2, \pi / 2+\delta / 2] \cap[-\pi / 2-\delta / 2,-\pi / 2+\delta / 2] .
$$

Therefore if (8.6) holds then

$$
|\operatorname{Im} z|>(1 / 2)|z| \geq r / 4=\left|\operatorname{Im} z_{r}\right| / 4 .
$$

We have proved assumptions of Lemma 8.9, thus

$$
r=\left|\operatorname{Im} z_{r}\right| \leq O\left(\log \left(2+\left|z_{r}\right|\right)\right)
$$

a contradiction.

Finally, we consider polynomials of two variables.

We call a polynomial $P(\omega)$-hyperbolic with respect to a vector $v$ [54] for a non-quasianalytic weight $\omega$ if $P_{p}(v) \neq 0$ (i.e., $v$ is non-characteristic) and $P(D)$ admits a fundamental solution $E \in \mathscr{D}_{(\omega)}^{\prime}\left(\mathbb{R}^{d}\right)$ with supp $E \subseteq \overline{H_{+}(v)}$, where

$$
H_{+}(v):=\left\{x \in \mathbb{R}^{d}:\langle x, v\rangle>0\right\} .
$$

A polynomial is $(\omega)$-hyperbolic if it is $(\omega)$-hyperbolic with respect to at least one non-characteristic vector. It is known that for $\omega(t)=\log (2+|t|)$, i.e., for classical distributions $\mathscr{D}^{\prime},(\omega)$-hyperbolicity is just the classical hyperbolicity [54, Remark 2.2(c)], [36, Def. 12.3.3, Th. 12.3.1,12.5.1]. We are ready to prove Theorem A (b). 
Theorem 8.13 Let $P \in \mathbb{C}\left[z_{1}, z_{2}\right]$ be an arbitrary polynomial. The following assertions are equivalent:

(a) $P(D): \mathscr{D}_{(\omega)}^{\prime}(\Omega, \mathscr{A}(U)) \rightarrow \mathscr{D}_{(\omega)}^{\prime}(\Omega, \mathscr{A}(U))$ is surjective for some open convex set $\Omega \subseteq \mathbb{R}^{2}$;

(b) $P(D): \mathscr{D}_{(\omega)}^{\prime}(\Omega) \rightarrow \mathscr{D}_{(\omega)}^{\prime}(\Omega)$ has a continuous linear right inverse for all open convex sets $\Omega \subseteq \mathbb{R}^{2}$;

(c) $P(D)$ is $(\omega)$-hyperbolic or, equivalently, $(\omega)$-hyperbolic with respect to all non-characteristic directions.

Remarks. 1. The paper [48] contains also the proof of $(b) \Leftrightarrow(c)$ for the classical space of distributions $\mathscr{D}^{\prime}(\Omega)$. Unfortunately the proof of necessity contained there is based on [48, Lemma 4.10] for which one can find a counterexample $P\left(z_{1}, z_{2}, z_{3}\right)=z_{1}+z_{2} z_{3}$. The general case $(\mathrm{b}) \Leftrightarrow(\mathrm{c})$ is contained in $[52$, Th. 5.14]

2. By [16, Th. 1], hyperbolicity with respect to all non-characteristic directions is equivalent to the fact that $P$ and the principal part $P_{p}$ are equally strong and $P_{p}$ is proportional to a product of linear functions with real coefficients.

Proof. (b) $\Rightarrow$ (a) Obvious. The equivalence of two conditions in part (c) is proved in [52, Th. 5.14]. (c) $\Rightarrow$ (b) is known [48, Th. 4.11], [52, Th. 5.14]. We will prove $(\mathrm{a}) \Rightarrow(\mathrm{c})$ :

Let us assume that $P$ is irreducible. Let $\vec{v}$ be a non-characteristic direction of $P$. Without loss of generality we may assume that $\vec{v}=(1,0)$.

Therefore, we may assume that

$$
P\left(z_{1}, z_{2}\right)=z_{1}^{m}+\sum_{j=0}^{m-1} e_{j}\left(z_{2}\right) z_{1}^{j} \quad \text { for all }\left(z_{1}, z_{2}\right) \in \mathbb{C}^{2} .
$$

Since $P_{p}$ is a homogenenous polynomial of two variables, we find $k, 0 \leq k \leq$ $m, 0 \neq \alpha_{j} \in \mathbb{C}$ for $1 \leq j \leq k$ (if $k>0$ ) such that

$$
P_{p}\left(z_{1}, z_{2}\right)=z_{1}^{m-k} \prod_{j=1}^{k}\left(z_{1}-\alpha_{j} z_{2}\right) \quad \text { for all }\left(z_{1}, z_{2}\right) \in \mathbb{C}^{2} .
$$

We assume that $1 \leq k<m$ (other cases are even simpler). By Theorem 8.6 and 8.5,

$$
P_{p}(D): \mathscr{D}_{(\omega)}^{\prime}\left(\mathbb{R}^{2}\right) \rightarrow \mathscr{D}_{(\omega)}^{\prime}\left(\mathbb{R}^{2}\right)
$$

has a continuous linear right inverse. By [48, 4.7], for every irreducible factor $Q$ of $P_{p}$

$$
\operatorname{dim}_{\mathbb{R}} V(Q) \cap \mathbb{R}^{2}=1,
$$

thus $\alpha_{j} \in \mathbb{R}$ for $1 \leq j \leq k$. 
Note that $V(P)$ can be represented via Puiseux expansion. Therefore there exists a constant $B>0$ such that

$$
V(P) \cap\left\{\left(z_{1}, z_{2}\right) \in \mathbb{C}^{2}:\left|z_{2}\right| \leq B\right\}
$$

is compact and

$$
V(P) \cap\left\{\left(z_{1}, z_{2}\right) \in \mathbb{C}^{2}:\left|z_{2}\right| \geq B\right\}
$$

is a union of disjoint branches $\left(W_{j}\right)$ each of them has the following expansion: there is $q_{j} \in \mathbb{N}$ such that for $\left|z_{2}\right| \geq B$ elements of $\left(z_{1}, z_{2}\right) \in W_{j}$ are of the form $\left(s_{j}\left(z_{2}\right), z_{2}\right)$,

$$
s_{j}\left(z_{2}\right)=\sum_{l=-\infty}^{q_{j}} a_{j l} z_{2}^{l / q_{j}} .
$$

Let us observe that

$$
\frac{1}{t}\left(s_{j}\left(t z_{2}\right), t z_{2}\right) \rightarrow\left(a_{j q_{j}} z_{2}, z_{2}\right) \in V\left(P_{p}\right), \quad \text { as } t \rightarrow \infty,
$$

for any $z_{2} \in \mathbb{C}$. Thus $a_{j q_{j}}$ must be equal to some $\alpha_{j}$ or to 0 and so it is real. It is easily seen that there exists a constant $B_{1}$ such that for any $z=\left(z_{1}, z_{2}\right) \in V(P)$

$$
|z| \leq B_{1}\left(\left|z_{2}\right|+1\right)
$$

Let us observe that for $B \leq\left|z_{2}\right| \leq B+1$

$$
\left|\operatorname{Im}\left(s_{j}\left(z_{2}\right)-a_{j q_{j}} z_{2}\right)\right| \leq C
$$

for some $C$ not dependent on $j$.

That is why the function $u$ defined for $z=\left(z_{1}, z_{2}\right) \in W_{j},\left|z_{2}\right| \geq B$ by the formula:

$$
u(z):=\max \left(\left|\operatorname{Im}\left(s_{j}\left(z_{2}\right)-a_{j q_{j}} z_{2}\right)\right|-C, 0\right)
$$

and $u(z):=0$ for $z=\left(z_{1}, z_{2}\right) \in V(P) \backslash W_{j}$ or $z=\left(z_{1}, z_{2}\right) \in V(P),\left|z_{2}\right| \leq B$ is a plurisubharmonic function on $V(P)$.

We choose $\iota, \lambda$ such that $\alpha:=\lambda / q_{\iota}$ is the biggest among the numbers $l / q_{j}$ such that $a_{j l} \neq 0, l<q_{j}, j$ arbitrary.

We will show that $t^{\alpha}=O(\omega(t))$ and this will be the most part of the proof below. Clearly it suffices to consider $0<\alpha<1$. If $t^{\alpha} \neq O(\omega(t))$ then there is a function $h:[0, \infty[\rightarrow[0, \infty[$ such that $\omega=o(h)$ and for some sequence $t_{j} \rightarrow \infty, h\left(t_{j}\right)=t_{j}^{\alpha}, \frac{h\left(t_{j}\right)}{\omega\left(t_{j}\right)} \rightarrow \infty$.

We start with some useful estimates for $u$. First,

$$
u(z) \leq D\left|z_{2}\right|^{\alpha}
$$

for all $z=\left(z_{1}, z_{2}\right) \in V(P)$. Since $a_{j q_{j}}$ are real

$$
u(z) \leq\left|\operatorname{Im} z_{1}\right|+D_{1}\left|\operatorname{Im} z_{2}\right| \quad \text { whenever } D_{1}:=\max _{j}\left|a_{j q_{j}}\right| \text {. }
$$


We need also estimates from below. If $a_{\iota \lambda} \notin \mathbb{R}$ then for $z_{2} \in \mathbb{R}$ :

$$
\left|\operatorname{Im}\left(s_{\iota}\left(z_{2}\right)-a_{\iota q_{\iota}} z_{2}\right)\right|=\left|\operatorname{Im} \sum_{l=-\infty}^{\lambda} a_{\iota l} z_{2}^{l / q_{\iota}}\right| \geq D_{1}\left|z_{2}\right|^{\alpha}
$$

for sufficiently big $\left|z_{2}\right|$. If $a_{\iota \lambda} \in \mathbb{R}, z_{2} \in \mathbb{R}$, then

$$
z_{2}^{\alpha}=\left|z_{2}\right|^{\alpha} \exp \left(i\left(\operatorname{Arg} z_{2}+2 \pi p\right) \alpha\right), \quad p=0,1, \ldots, q_{\iota}-1,
$$

where $\operatorname{Arg} z_{2}=0$ for $z_{2}>0$ and $=\pi$ for $z_{2}<0$. Choosing appropriately $p$ and $\operatorname{Arg} z_{2}$ we get for all suitable big $\left|z_{2}\right|$ that

$$
\left|\operatorname{Im} a_{\iota \lambda} z_{2}^{\alpha}\right| \geq D_{3}\left|z_{2}\right|^{\alpha}
$$

Summarizing, there is a constant $E>0$ such that for big real $z_{2}$ we have

$$
\left|\operatorname{Im}\left(s_{\iota}\left(z_{2}\right)-a_{\iota q_{\iota}} z_{2}\right)\right| \geq E\left|z_{2}\right|^{\alpha} \quad \text { for suitable choice of } s_{\iota}\left(z_{2}\right) .
$$

By Corollary 8.2, $V(P)$ has $\mathrm{IPL}_{+}^{0}\left(\mathbb{R}^{2}, \omega\right)$ in the form described in Corollary 7.9 (ii). Let us fix $w \in \mathbb{R}, B \leq|w|=R, \mu=4 A \geq 2$. Define for $z=\left(z_{1}, z_{2}\right) \in V(P)$ :

$$
\tilde{u}(z)=\left\{\begin{array}{l}
\max \left(\mu u(z)-\frac{R}{2} \operatorname{Re}\left[\left(\frac{z_{2}-w}{R}\right)^{2}\right],|\operatorname{Im} z|\right) \quad \text { for }\left|z_{2}-w\right| \leq R \\
|\operatorname{Im} z| \quad \text { otherwise on } V(P)
\end{array}\right.
$$

For $\left|z_{2}-w\right|=R$ we have by $(8.9)$

$$
\begin{aligned}
\mu u(z) & -\frac{R}{2} \operatorname{Re}\left[\left(\frac{z_{2}-w}{R}\right)^{2}\right]= \\
& =\mu u(z)+\frac{R}{2}\left[\left|\operatorname{Im}\left(\frac{z_{2}-w}{R}\right)\right|^{2}-\left|\operatorname{Re}\left(\frac{z_{2}-w}{R}\right)\right|^{2}\right] \leq \\
& \leq \mu D\left|z_{2}\right|^{\alpha}+\left|\operatorname{Im} z_{2}\right|-R / 2 \leq|\operatorname{Im} z|
\end{aligned}
$$

for sufficiently big $R$. Hence $\tilde{u}$ is a plurisubharmonic function on $V(P)$. Analogously, we obtain for $z \in V(P)$

$$
\tilde{u}(z) \leq|\operatorname{Im} z|+\mu D 2^{\alpha} R^{\alpha} .
$$

We take $\rho:=\left(\mu+\mu D_{1}+3\right) / 2, m$ arbitrary, $\theta=E /\left(2^{\alpha+2} D\right)$. We choose by Lemma 2.2 a weight $\sigma, \omega(z) \leq \sigma(z), \omega=o(\sigma), \sigma=o(h)$ and we construct a plurisubharmonic function $\varphi$ such that

$$
-C_{k} \sigma(z) \leq \varphi(z) \leq|\operatorname{Im} z|-2 k(\theta) \sigma(z) .
$$


We define

$$
v(z):=(\tilde{u}(z)+\varphi(z)) / 2 .
$$

Then, by (8.12)

$$
v(z) \leq|\operatorname{Im} z|-k(\theta) \sigma(z)+\mu D 2^{\alpha-1} R^{\alpha} .
$$

Clearly $v \in \operatorname{PSH}(V, B(0,1))$ and

$$
v(z) \leq|\operatorname{Im} z|-n \omega(z)+\mu D R^{\alpha} 2^{\alpha-1} .
$$

On the other hand, by (8.10) since for $\left|z_{2}-w\right| \leq R$ we have

$$
-\frac{R}{2} \operatorname{Re}\left[\left(\frac{z_{2}-w}{R}\right)^{2}\right] \leq\left|\operatorname{Im} z_{2}\right|
$$

we get

$$
\tilde{u}(z) \leq \mu\left|\operatorname{Im} z_{1}\right|+\mu D_{1}\left|\operatorname{Im} z_{2}\right|+\left|\operatorname{Im} z_{2}\right|+|\operatorname{Im} z|
$$

therefore,

$$
v(z) \leq \rho|\operatorname{Im} z|-k(\theta) \omega(z)
$$

By $\operatorname{IPL}_{+}^{0}\left(\mathbb{R}^{2}, \omega\right)$

$$
v(z) \leq A|\operatorname{Im} z|-m \omega(z)+\theta \mu D R^{\alpha} 2^{\alpha-1}
$$

and

$$
\tilde{u}(z) \leq 2 A|\operatorname{Im} z|-2 m \omega(z)+2^{\alpha} \theta \mu D R^{\alpha}+C_{k} \sigma(z) .
$$

Taking $z=\left(z_{1}, w\right) \in W_{j}$ we get $\mu u(z) \leq \tilde{u}(z)$ and

$$
u(z) \leq \frac{1}{2}|\operatorname{Im} z|+2^{\alpha} \theta D R^{\alpha}+C_{k} \sigma(z) / \mu .
$$

Moreover, $u(z)=\max (|\operatorname{Im} z|-C, 0)$ since $z_{2}=w \in \mathbb{R}, a_{j q_{j}} \in \mathbb{R}$, hence either $|\operatorname{Im} z| \leq C$ or

$$
|\operatorname{Im} z|=u(z)+C \leq \frac{1}{2}|\operatorname{Im} z|+2^{\alpha} \theta D R^{\alpha}+C_{k} \sigma(z) / \mu+C .
$$

Therefore

$$
|\operatorname{Im} z| \leq 2^{\alpha+1} \theta D R^{\alpha}+2 C_{k} \sigma(z) / \mu+2 C .
$$

Using (8.11) and $|w|=R$ we get by choosing suitable $z_{1}$

$$
E R^{\alpha} \leq 2^{\alpha+1} \theta D R^{\alpha}+2 C_{k} \sigma(z) / \mu+2 C .
$$

Since $\theta=E /\left(2^{\alpha+2} D\right)$,

$$
R^{\alpha} \leq \frac{4 C_{k}}{\mu E} \sigma(z)+\frac{4 C}{E} .
$$

By (8.8) $\sigma(z)=O\left(\sigma\left(z_{2}\right)\right)=o(h(R))$; a contradiction.

We have proved that $t^{\alpha}=O(\omega(t))$. 
Again applying (8.7) and the fact that all $a_{j q_{j}}$ are real we get for $z=$ $\left(z_{1}, z_{2}\right) \in V(P), z_{2} \in \mathbb{R}$

$$
\left|\operatorname{Im} z_{1}\right| \leq F\left(1+\omega\left(z_{2}\right)\right)
$$

for some constant $F$ which implies $(\omega)$-hyperbolicity with respect to $\vec{v}$ by the definition (see [54, Prop. 2.7]).

For reducible $P$ it follows that each of its irreducible factors is $(\omega)$ hyperbolic with respect to each non-characteristic direction. The conclusion follows by [54, Lemma 2.5].

Clearly, a careful reader will find several further results on the parameter dependence following our theory, for instance, for ultradifferential dependence instead of real analytic dependence — we omit details.

\section{Open problems}

We collect some open problems suggested by the theory described in this paper. The first main problem is the following:

Problem 9.1 Is it true that for arbitrary polynomial $P$ the positive solution of the real analytic parameter dependence problem for $P(D): \mathscr{D}^{\prime}(\Omega) \rightarrow$ $\mathscr{D}^{\prime}(\Omega)$ is equivalent to the existence of continuous linear right inverse for the same operator?

This problem is exactly equivalent to the following question (comp. [6, Problem 9.5]):

Problem 9.2 Can we characterize existence of a continuous linear right inverse for $P(D): \mathscr{D}^{\prime}(\Omega) \rightarrow \mathscr{D}^{\prime}(\Omega)$ by the dual interpolation estimate for the kernel of $P(D)$ ?

There are two tempting particular cases of Problem 9.1: $\Omega=H_{+}$a half space and $\Omega$ an open bounded set with $C^{1}$-boundary. By Theorem 8.5 and [48, Cor.2.10], a positive solution of the first case implies a positive solution for the second one. In view of [48, Prop. 3.2] the problem reduces to the question of hyperbolicity with respect to the normal direction to the boundary of a $H_{+}$.

By [23, Th. 1, Th. 3] (comp. [22]), $P(D): \mathscr{D}^{\prime}(\Omega) \rightarrow \mathscr{D}^{\prime}(\Omega)$ has a continuous linear right inverse if and only if the kernel ker $P(D)$ is a strict PLS-space. The same holds for any operator $T: \mathscr{D}^{\prime}(\Omega) \rightarrow \mathscr{D}^{\prime}(\Omega)$ with a translation invariant kernel or the so-called $s^{\prime}$-friendly kernel. Therefore Problem 9.1 is very close to the conjecture that every translation invariant subspace of $\mathscr{D}_{(\omega)}^{\prime}(\Omega)$ with $(P \overline{\bar{\Omega}})$ (or, with the dual interpolation estimate) is strict. We do not know any counterexample although there is an example of 
a PLS-Köthe sequence space which is $s^{\prime}$-friendly non-strict subspace of $\mathscr{D}^{\prime}$ with $(P \overline{\bar{\Omega}})$ and which is neither LB-space nor a Fréchet space.

Of course, one can consider Problem 9.1 and Problem 9.2 for ultradistributions $\mathscr{D}_{(\omega)}^{\prime}(\Omega)$ and ultradifferentiable functions $\mathscr{E}_{\{\omega\}}(\Omega)$ as well.

There are interesting cases not covered by Theorem 5.2 .

Problem 9.3 Characterize $\operatorname{Proj}^{1} X \varepsilon E=0$ for a $P L N$-space $X$ and a $P L S$ space $E$ in terms of conditions $(T)$ or $\left(T_{\varepsilon}\right)$.

The problem is open even for Fréchet Schwartz $E$.

A truly challenging problem is to characterize in terms of the symbol kernels of linear partial differential operators with variable coefficients on $\mathscr{D}^{\prime}(\Omega)$ or $\mathscr{A}(\Omega)$ which have the dual interpolation estimate for small $\theta$ since this would solve the real analytic parameter dependence problem for partial differential operators with variable coefficients via Corollary 6.4. Creating a suitable theory would require a nice description of the kernel — so it would be the analogue of the fundamental principle for partial differential operators with variable coefficients. It seems that we are very far from that.

Acknowledgements. The author is very indebted to many colleagues for discussions on the topics of that paper and remarks on it: J. Bonet, L. Frerick, M. Jasiczak, M. Langenbruch, R. Meise, D. Vogt. Special thanks to Dietmar Vogt for his warm hospitality during the author's stay in Wuppertal in Autumn 2006 as well as during many earlier visits.

\section{References}

[1] Alexiewicz, A. ANd Orlicz, W.: On analytic vector-valued functions of a real variable. Studia Math. 12 (1951), 108-111.

[2] Bergh, J. And Löfström, J.: Interpolation spaces. An introduction. Grundlehren der Mathematischen Wissenschaften 223. Springer-Verlag, Berlin-New York, 1976.

[3] Bierstedt, K. D. And Meise, R.: Induktive Limites gewichteter Räume stetiger und holomorpher Funktionen. J. Reine Angew. Math. 282 (1976), $186-220$.

[4] Bonet, J. And Domański, P.: Real analytic curves in Fréchet spaces and their duals. Monatsh. Math. 126 (1998), 13-36.

[5] Bonet, J. And Domański, P.: Parameter dependence of solutions of partial differential equations in spaces of real analytic functions. Proc. Amer. Math. Soc. 129 (2001), 495-503.

[6] Bonet, J. And Domański, P.: Parameter dependence of solutions of differential equations on spaces of distributions and the splitting of short exact sequences. J. Funct. Anal. 230 (2006), 329-381. 
[7] Bonet, J. And Domański, P.: The structure of spaces of quasianalytic functions of Roumieu type. Arch. Math. (Basel) 89 (2007), 430-441.

[8] Bonet, J. And Domański, P.: The splitting of exact sequences of PLSspaces and smooth dependence of solutions of linear partial differential equations. Adv. Math. 217 (2008), 561-585.

[9] Bonet, J., Meise, R. And Melikhov, S. N.: A comparison of two different ways to define classes of ultradifferentiable functions. Bull. Belg. Math. Simon Stevin 14 (2007), 425-444.

[10] Braun, R. W. And Meise, R.: Generalized Fourier expansions for zerosolutions of surjective convolution operators on $\mathscr{D}_{\{\omega\}}^{\prime}(\mathbb{R})$. Arch. Math. (Basel) 55 (1990), 55-63.

[11] Braun, R. W., Meise, R. and Taylor, B. A.: Ultradifferentiable functions and Fourier analysis. Results Math. 17 (1990), 207-237.

[12] Braun, R. W., Meise, R. and Taylor, B. A.: Characterization of the linear partial differential equations that admit solution operators on Gevrey classes. J. Reine Angew. Math. 588 (2005), 169-220.

[13] Browder, F. E.: Analyticity and partial differential equations. I. Amer. J. Math. 84 (1962), 666-710.

[14] Cartan, H.: Variétés analytiques réelles et variétés analytiques complexes. Bull. Soc. Math. France 85 (1957), 77-99.

[15] Cascales, B. and Orinuela, J.: Metrizability of precompact subsets in (LF)-spaces. Proc. Roy. Soc. Edinburgh Sect. A 103 (1986), 293-299.

[16] De Christoforis, M. L.: Soluzioni con lacune di certi operatori differenziali lineari. Rend. Accad. Naz. Sci. XL Mem. Mat. (5) 8 (1984), 137-142.

[17] Dierolf, S.: On spaces of continuous linear mappings between locally convex spaces. Note Mat. 5 (1985), 147-255.

[18] Domański, P.: Classical PLS-spaces: spaces of distributions, real analytic functions and their relatives. In Orlicz centenary volume, 51-70. Banach Center Publ. 64. Polish Acad. Sci., Warsaw, 2004.

[19] Domański, P. and Langenbruch, M.: Composition operators on spaces of real analytic functions. Math. Nachr. 254-255 (2003), 68-86.

[20] Domański, P. And Langenbruch, M.: Vector valued hyperfunctions and boundary values of vector valued harmonic and holomorphic functions. Publ. Res. Inst. Math. Sci. 44 (2008), no. 4, 1097-1142.

[21] Domański, P. And MastyŁo, M.: Characterization of splitting for Fréchet-Hilbert spaces via interpolation. Math. Ann. 339 (2007), 317-340.

[22] Domański, P. And Vogt, D.: A splitting theory for the space of distributions. Studia Math. 140 (2000), 57-77.

[23] Domański, P. And Vogt, D.: Distributional complexes split for positive dimensions. J. Reine Angew. Math. 522 (2000), 63-79.

[24] Domański, P. And Vogt, D.: The space of real analytic functions has no basis. Studia Math. 142 (2000), 187-200. 
[25] Federer, H.: Geometric measure theory. Die Grundlehren der mathematischen Wissenschaften 153. Springer-Verlag, New York, 1969.

[26] Franken, U.: On the equivalence of holomorphic and plurisubharmonic Phragmén-Lindelöf principles. Michigan Math. J. 42 (1995), no. 1, 163-173.

[27] Franken, U. and Meise, R.: Generalized Fourier expansions for zerosolutions of surjective convolution operators on $\mathscr{D}^{\prime}(\mathbb{R})$ and $\mathscr{D}_{(\omega)}^{\prime}(\mathbb{R})$. Note Mat. 10 (1990), suppl. 1, 251-272 (1992).

[28] Frerick, L.: A splitting theorem for nuclear Fréchet spaces. In Functional analysis (Trier, 1994), 163-167. Walter de Gruyter, Berlin, 1996.

[29] Frerick, L., Kunkle, D. And Wengenroth, J.: The projective limit functor for spectra of webbed spaces. Studia Math. 158 (2003), 117-129.

[30] Frerick, L. And Wengenroth, J.: A sufficient condition for the vanishing of the derived projective limit functor. Arch. Math. (Basel) 67 (1996), 296-301.

[31] Grauert, H.: On Levi's problem and the imbedding of real analytic manifolds. Ann. of Math. (2) 68 (1958), 460-472.

[32] Grothendieck, A.: Produits tensoriels topologiques et espaces nucléaires. Mem. Amer. Math. Soc. 16 (1955).

[33] Hansen, S.: Das Fundamentalprinzip für Systeme linearer partieller Differentialgleichungen mit konstanten Koeffizienten. Habilitationsschrift. Paderborn, 1982.

[34] Hirsch, M. W.: Differential topology. Graduate Texts in Mathematics 33. Springer-Verlag, New York-Heidelberg, 1976.

[35] Hörmander, L.: On the existence of real analytic solutions of partial differential equations with constant coefficients. Invent. Math. 21 (1973), 151-182.

[36] Hörmander, L.: The analysis of linear partial differential operators. Springer, Berlin 1983.

[37] Jarchow, H.: Locally convex spaces. B. G. Teubner, Stuttgart, 1981.

[38] Klimek, M.: Pluripotential theory. London Mathematical Society Monographs New Series 6. Oxford University Press, New York, 1991.

[39] Komatsu, H.: Ultradistributions. I. Structure theorems and characterization. J. Fac. Sci. Univ. Tokyo Sect. IA. Math. 20 (1973), 25-105.

[40] Kriegl, A. And Michor, P. W.: The convenient setting for real analytic mappings. Acta Math. 165 (1990), 105-159.

[41] Kriegl, A. And Michor, P. W.: The convenient setting of global analysis. Mathematical Surveys and Monographs 53. American Mathematical Society, Providence, RI, 1997.

[42] Langenbruch, M.: Continuous linear right inverses for convolution operators in spaces of real analytic functions. Studia Math. 110 (1994), 65-82.

[43] Luecking, D. H. And Rubel, L. A.: Complex analysis. A functional analysis approach. Universitext. Springer-Verlag, New York, 1984. 
[44] Mantlik, F.: Partial differential operators depending analytically on a parameter. Ann. Inst. Fourier (Grenoble) 41 (1991), 577-599.

[45] Mantlik, F.: Linear equations depending differentiably on a parameter. Integral Equations Operator Theory 13 (1990), 231-250.

[46] Mantlik, F.: Fundamental solutions for hypoelliptic differential operators depending analytically on a parameter. Trans. Amer. Math. Soc. 334 (1992), 245-257.

[47] Martineau, A.: Sur la topologie des espaces de fonctions holomorphes. Math. Ann. 163 (1966), 62-88.

[48] Meise, R., Taylor, B. A. and Vogt, D.: Characterization of the linear partial differential operators with constant coefficients that admit a continuous linear right inverse. Ann. Inst. Fourier (Grenoble) 40 (1990), 619-655.

[49] Meise, R., Taylor, B. A. and Vogt, D.: Equivalence of analytic and plurisubharmonic Phragmén-Lindelöf conditions. In Several complex variables and complex geometry, Part 3 (Santa Cruz, CA, 1989), 287-308. Proc. Sympos. Pure Math. 52, Part 3. Amer. Math. Soc., Providence, RI, 1991.

[50] Meise, R., Taylor, B. A. And Vogt, D.: Continuous linear right inverses for partial differential operators with constant coefficients and PhragménLindelöf conditions. In: Functional analysis (Essen, 1991), 357-389. Lecture Notes in Pure and Appl. Math. 150. Dekker, New York, 1994.

[51] Meise, R., TAYlor, B. A. And Vogt, D.: Extremal plurisubharmonic functions of linear growth on algebraic varieties. Math. Z. 219 (1995), $515-537$.

[52] Meise, R., Taylor, B. A. And Vogt, D.: Continuous linear right inverses for partial differential operators on non-quasianalytic classes and on ultradistributions. Math. Nachr. 180 (1996), 213-242.

[53] Meise, R., Taylor, B. A. And Vogt, D.: Continuous linear right inverses for partial differential operators of order 2 and fundamental solutions in half spaces. Manuscripta Math. 90 (1996), 449-464.

[54] Meise, R., Taylor, B. A. And Vogt, D.: $\omega$-Hyperbolicity of linear partial differential operators with constant coefficients. In Complex analysis, harmonic analysis and applications (Bordeaux, 1995), 157-182. Pitman Res. Notes Math. Ser. 347. Longman, Harlow, 1996.

[55] Meise, R., Taylor, B. A. And Vogt, D.: Phragmén-Lindelöf principles on algebraic varieties. J. Amer. Math. Soc. 11 (1998), 1-39.

[56] Meise, R. And Vogt, D.: Characterization of convolution operators on spaces of $C^{\infty}$-functions admitting a continuous linear right inverse. Math. Ann. 279 (1987), 141-155.

[57] Meise, R. And Vogt, D.: Introduction to functional analysis. Oxford Graduate Texts in Mathematics 2. The Clarendon Press, Oxford University Press, New York, 1997. 
[58] Meyer, T.: Surjectivity of convolution operators on spaces of ultradifferentiable functions of Roumieu type. Studia Math. 125 (1997), 101-129.

[59] Mitjagin, B. S. and Henkin, G. M.: Linear problems of complex analysis. Uspekhi Mat. Nauk 26 (1971), no. 4 (160), 93-152 (Russian). English translation Russian Math. Surv. 26 (1971), 99-164.

[60] Narasimhan, R.: Analysis on real and complex manifolds. Advanced Studies in Pure Mathematics 1. North-Holland, Amsterdam, 1968.

[61] Ovchinnikov, V. I.: The methods of orbits in interpolation theory. Math. Rep. 1 (1984), i-x and 349-515.

[62] Palamodov, V. P.: Linear differential operators with constant coefficients. Nauka, Moscow, 1967 (Russian). English transl.: Die Grundlehren der mathematischen Wissenschaften 168. Springer-Verlag, New York-Berlin, 1970.

[63] Palamodov, V. P.: Functor of projective limit in the category of topological vector spaces. Mat. Sb. 75 (1968), 567-603 (Russian). English translation: Math. USSR Sbornik 17 (1972), 289-315.

[64] Palamodov, V. P.: Homological methods in the theory of locally convex spaces. Uspekhi Mat. Nauk 26 (1971), no. 1 (157), 3-66 (Russian). English transl.: Russian Math. Surveys 26 (1) (1971), 1-64.

[65] Palamodov, V.P.: On a Stein manifold the Dolbeault complex splits in positive dimensions. Mat. Sb. (N.S.) 88 (1972), 287-315 (Russian). English transl.: Math. USSR Sbornik 17 (1972) 289-315.

[66] Petzsche, H. J.: Some results of Mittag-Leffler-type for vector valued functions and spaces of class $\mathcal{A}$. In Functional analysis: surveys and recent results, II (Proc. Second Conf. Functional Anal., Univ. Paderborn, Paderborn, 1979), 183-204. North-Holland Math. Stud. 38. North-Holland, AmsterdamNew York, 1980.

[67] PiszczeK, K.: On a property of PLS-spaces inherited by their tensor products. Bull. Belg. Math. Soc. Simon Stevin (to appear).

[68] Poly, J. B.: Sur les opérateurs différentiels et les morphismes directs. C.R. Acad. Sci. Paris Sér. A-B 270 (1970), A647-A649.

[69] Rodino, L.: Linear partial differential operators in Gevrey spaces. World Scientific Publishing, River Edge, NJ, 1993.

[70] Rösner, T.: Surjektivität partieller Differentialoperatoren auf quasianalytischen Roumieu-Klassen. Dissertation, Düsseldorf, 1997.

[71] Schwartz, L.: Théorie des distributions à valeurs vectorielles. I, II. Ann. Inst. Fourier 7 (1957), 1-141, 8 (1958), 1-209.

[72] TrÈves, F.: Un théorème sur les équations aux dérivées partielles à coefficients constants dépendant de paramètres. Bull. Soc. Math. France 90 (1962), 473-486.

[73] TrÈves, F.: Fundamental solutions of linear partial differential equations with constant coefficients depending on parameters. Amer. J. Math. 84 (1962), 561-577. 
[74] Varol, O.: On the derived tensor product functors for (DF)- and Fréchet spaces. Studia Math. 180 (2007), 41-71.

[75] Vogt, D.: Eine Charakterisierung der Potenzreihenräume von endlichem Typ und ihre Folgerungen. Manuscripta Math. 37 (1982), 269-301.

[76] Vogt, D.: Sequence space representations of spaces of test functions and distributions. In Functional analysis, holomorphy, and approximation theory (Rio de Janeiro, 1979), 405-443. Lecture Notes Pure Appl. Math. 83. Marcel Dekker, New York 1983.

[77] Vogt, D.: Some results on continuous linear maps between Fréchet spaces. In Functional analysis: surveys and recent results, III (Paderborn, 1983), 349-381. North-Holland Math. Stud. 90. North-Holland, Amsterdam, 1984.

[78] Vogt, D.: Lectures on projective spectra of DF-spaces. Seminar Lectures, AG Funktionalanalysis, Düsseldorf/Wuppertal, 1987.

[79] Vogt, D.: Topics on projective spectra of LB-spaces. In Advances in the theory of Fréchet Spaces (Istanbul, 1988), 11-27. NATO Adv. Sci. Inst. Ser. C Math. Phys. Sci. 287. Kluwer Acad. Publ., Dordrecht, 1989.

[80] Vogt, D.: Invariants and spaces of zero solutions of linear partial differential operators. Arch. Math. (Basel) 87 (2006), 163-171.

[81] Vogt, D.: Extension operators for real analytic functions on compact subvarieties of $\mathbb{R}^{d}$. J. Reine Angew. Math. 606 (2007), 217-233.

[82] Vogt, D. And Wagner, M. J.: Charakterisierung der Unterräume und Quiotientenräume der nuklearen stabilen Potenzreihenräume von unendlichem Typ. Studia Math. 70 (1981), 63-80.

[83] Wengenroth, J.: Derived functors in functional analysis. Lecture Notes in Mathematics 1810. Springer-Verlag, Berlin, 2003.

Recibido: 2 de julio de 2008

Paweł Domański

Faculty of Mathematics and Comp. Sci.

A. Mickiewicz University Poznań and Institute of Mathematics

Polish Academy of Sciences (Poznań branch)

Umultowska 87, 61-614 Poznań, Poland domanski@amu.edu.pl

The research of the author was supported in years 2007-2010 by Ministry of Science and Higher Education, Poland, grant no. NN201 2740 33. Part of the research was done during author's stay at Bergische Universität Wuppertal supported by Alexander von Humboldt Stiftung. 